\title{
Hard x-ray photoelectron spectroscopy: a snapshot of the state-of-the-art in 2020
}

\author{
Curran Kalha ${ }^{1,13}$ (), Nathalie K Fernando ${ }^{1,13}{ }^{\odot}$, Prajna Bhatt ${ }^{1}{ }^{\oplus}$, Fredrik O L \\ Johansson $^{2} \odot{ }^{\circ}$, Andreas Lindblad ${ }^{2} \odot$, Håkan Rensmo ${ }^{2}$, León Zendejas

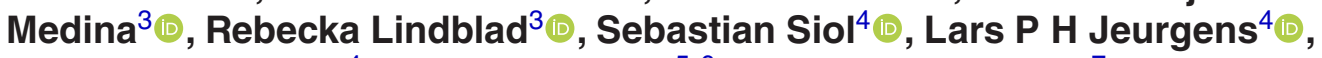 \\ Claudia Cancellieri ${ }^{4}{ }^{\circ}$, Kai Rossnage ${ }^{5,6}{ }^{0}$, Katerina Medjanik ${ }^{7}$, Gerd \\ Schönhense ${ }^{7}{ }^{\circ}$, Marc Simon $^{8}{ }^{\circ}$, Alexander X Gray ${ }^{9}{ }^{\circ}$, Slavomír

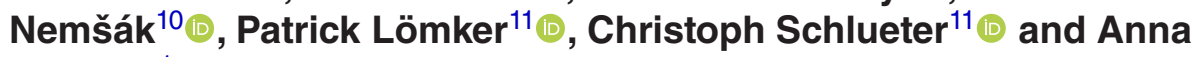 \\ Regoutz $^{1, *}$ \\ ${ }^{1}$ Department of Chemistry, University College London, 20 Gordon Street, London, WC1H 0AJ, United \\ Kingdom \\ 2 Department of Physics and Astronomy, Uppsala University, Box 516, 75120 Uppsala, Sweden \\ ${ }^{3}$ Department of Chemistry—Ångström Laboratory, Uppsala University, Box 538, SE-75121, Uppsala, \\ Sweden \\ ${ }^{4}$ Empa, Swiss Federal Laboratories for Materials Science and Technology, Laboratory for Joining \\ Technologies and Corrosion, Dübendorf, Switzerland \\ ${ }^{5}$ Institute of Experimental and Applied Physics, Kiel University, 24098 Kiel, Germany \\ ${ }^{6}$ Ruprecht Haensel Laboratory, Deutsches Elektronen-Synchrotron DESY, 22607 Hamburg, Germany \\ 7 Johannes Gutenberg Universität, Institut für Physik, 55128 Mainz, Germany \\ ${ }^{8}$ Sorbonne Université, CNRS, Laboratoire de Chimie Physique-Matière et Rayonnement, LCPMR, \\ F-75005 Paris, France \\ 9 Department of Physics, Temple University, Philadelphia, PA 19122, United States of America \\ 10 Advanced Light Source, Lawrence Berkeley National Laboratory, Berkeley, CA 94720, United States of \\ America \\ 11 Deutsches Elektronen-Synchrotron DESY, 22607 Hamburg, Germany \\ E-mail: a.regoutz@ucl.ac.uk
}

Received 21 December 2020, revised 3 February 2021

Accepted for publication 1 March 2021

Published 13 May 2021

\section{Abstract}

Hard x-ray photoelectron spectroscopy (HAXPES) is establishing itself as an essential technique for the characterisation of materials. The number of specialised photoelectron spectroscopy techniques making use of hard $\mathrm{x}$-rays is steadily increasing and ever more complex experimental designs enable truly transformative insights into the chemical, electronic, magnetic, and structural nature of materials. This paper begins with a short historic perspective of HAXPES and spans from developments in the early days of photoelectron spectroscopy to provide an understanding of the origin and initial development of the technique to state-of-the-art instrumentation and experimental capabilities. The main motivation for and focus of this paper is to provide a picture of the technique in 2020 ,

*Author to whom any correspondence should be addressed.

${ }^{13}$ Both authors contributed equally to the manuscript. 
including a detailed overview of available experimental systems worldwide and insights into a range of specific measurement modi and approaches. We also aim to provide a glimpse into the future of the technique including possible developments and opportunities.

Keywords: photoelectron spectroscopy, hard x-ray photoelectron spectroscopy, photoemission spectroscopy

S Supplementary material for this article is available online

(Some figures may appear in colour only in the online journal)

\section{Introduction and historic perspective}

All photoelectron spectroscopy (PES) is based on the fundamental principle of the photoelectric effect [1-3], which was explained in terms of the photon concept by Einstein and Rutherford [4, 5]. The photoelectric effect describes how light that hits a material gives rise to the emission of electrons, i.e. photoelectrons. From this foundation it took decades of experimental work to develop an experimental technique that could capitalise on this effect to obtain physical and chemical information. It was only with techniques developed for nuclear spectroscopy by Siegbahn and his group, that the first steps of PES were taken $[6,7]$. The group of Siegbahn initially aimed for correcting nuclear energies from effects of inner shell core electron excitations. Such corrections required the development of electron spectrometers for high energies and in fact the first spectrometers were limited to energies of a few $\mathrm{keV}$ and above. Their first core photoelectron spectrum showing discrete lines, detected the $\mathrm{Cu} 1 s$ photoelectrons excited by $\mathrm{Mo} \mathrm{K} \alpha_{1}(h \nu=17.479 \mathrm{keV})$ and Mo K $\alpha_{2}(h \nu=17.374 \mathrm{keV})$ $\mathrm{X}$-rays [8].

In this context the early development of PES coincides with the emergence of hard x-ray photoelectron spectroscopy (HAXPES). From the beginning careful energy calibration, linked to the understanding of work function, Fermi level, and control over charging, was realised as a very important aspect. Such control and the use of hard x-ray set-ups allowed for the early indication of the chemical shift $[9,10]$, the existence of which was firmly established together with quantitative insights from the use of $\mathrm{Cu} \mathrm{K} \alpha(h \nu=8.046 \mathrm{keV})$ and $\mathrm{Cr} \mathrm{K} \alpha(h \nu=5.415 \mathrm{keV})[11,12]$. During this time the use of electron spectroscopy as a tool for chemical analysis was established [13], and started to spread over the world [14], with much of this work being performed using hard x-rays. Thus, early parts of modern photoelectron spectroscopy were accomplished using energies that today would use the acronym HAXPES.

HAXPES is generally defined as X-ray photoelectron spectroscopy (XPS) which uses x-ray energies above $2 \mathrm{keV}$, as historically beyond $2 \mathrm{keV}$ crystal monochromators replaced grating monochromators used in the soft $\mathrm{x}$-ray regime. Although developments in grating technology have led to some grating monochromators being able to go up to $5 \mathrm{keV}$, the definition remains in place. A range of abbreviations is in use for the technique with HAXPES, which was first used in 2005 [15, 16], being the most widely established and others including HXPS, HXPES, HX-PES, and HIKE. To date, HAXPES has been applied in many scientific areas from fundamental atomic, molecular, and condensed matter physics, to surface and interface science, and technologically important areas like catalysis, electrochemistry, energy materials, including batteries, fuel cells, and photovoltaics, and electronic devices.

In standard laboratory-based soft X-ray photoelectron spectroscopy (SXPS or XPS) monochromated $\mathrm{Al} \mathrm{K} \alpha$ and $\mathrm{Mg} \mathrm{K} \alpha$, at $h \nu=1486.7 \mathrm{eV}$ and $1253.6 \mathrm{eV}$, respectively, are most widely used as they can be produced with high intensities and have intrinsically narrow line widths to enable good energy resolution necessary for both core and valence state spectroscopy. In the first published paper by Kai Siegbahn on electron spectroscopy for chemical analysis (ESCA), which we now commonly know as XPS, he points out that for all elements of the periodic table 'one or several narrow atomic levels' can be found and measured using $\mathrm{Al} \mathrm{K} \alpha$ or $\mathrm{Mg}$ $\mathrm{K} \alpha \mathrm{x}$-rays. He goes on to state that, if one is interested in deeper atomic levels, these can be excited with $\mathrm{Cr}\left(h \nu_{\mathrm{K} \alpha}\right.$ $=5.415 \mathrm{keV}), \mathrm{Cu}\left(h \nu_{\mathrm{K} \alpha}=8.046 \mathrm{keV}\right), \mathrm{Ag}\left(h \nu_{\mathrm{L} \alpha}\right.$ $=2.984 \mathrm{keV})$, or $\mathrm{W}\left(h \nu_{\mathrm{L} \alpha}=8.398 \mathrm{keV}\right)$, but that in his opinion $\mathrm{Al} \mathrm{K} \alpha$ or $\mathrm{Mg} \mathrm{K} \alpha$ are preferable due to their line widths. This point of view is understandable as $\mathrm{Al}$ and $\mathrm{Mg}$ have line widths of below $1 \mathrm{eV}$, while the hard x-ray sources generally have widths above $2 \mathrm{eV}$. Nevertheless, very early HAXPES studies were performed using $\mathrm{Cu} \mathrm{K} \alpha$ in the 1960s [14, 17].

Beyond these initial proof of concept experiments at laboratory sources, it is the advent of synchrotron sources that really catalyzed the development of HAXPES. The first reported synchrotron-based HAXPES measurements were performed by Lindau, Pianetta et al at the Stanford Synchrotron Radiation Project using synchrotron radiation from the Stanford Positron Electron Accelerator Ring (SPEAR) facility $[18,19]$. This first experiment reported the $\mathrm{Au} 4 f$ core level measured at $8 \mathrm{keV}$ and with an impressive energy resolution of $0.25 \mathrm{eV}$ achieved by combining two $\mathrm{Si}(220)$ crystals and a $\mathrm{Si}(440)$ channel-cut crystal. Following on from this first experiment developments both in the USA and at HASYLAB, Hamburg, Germany, in the 1980s and 1990s led to a number of core level HAXPES 
Table 1. HAXPES beamlines currently in operation at synchrotrons worldwide. The information given includes the name of the beamline, the synchrotron, the host country, the type of source used [insertion device (ID) or bending magnet (BM)], the energy range $h \nu$ available at the beamline, the best energy resolution $\Delta E$ and at what energy $h \nu$ this is accessible (in brackets), the x-ray $h \nu$ spot size at the sample position, and any publications directly relating to the beamlines.

\begin{tabular}{lccccccc}
\hline Beamline & Synchrotron & Country & Source & $h \nu / \mathrm{keV}$ & $\Delta E(h \nu) / \mathrm{meV}(\mathrm{keV})$ & $h \nu$ spot/ $\mu \mathrm{m}^{2}$ & References \\
\hline BM25 SpLine & ESRF & France & ID & $3-40$ & $80(5)$ & $100 \times 100$ & {$[49]$} \\
GALAXIES & SOLEIL & France & ID & $2.3-12$ & $80(4)$ & $80 \times 30$ & {$[50-52]$} \\
EMIL & BESSY II & Germany & ID & $0.08-10$ & $\mathrm{a}$ & $100 \times 20$ & {$[53]$} \\
KMC-1 & BESSY II & Germany & BM & $2-12$ & $250(2-6)$ & $100 \times 50$ & {$[54,55]$} \\
P22 & PETRA III & Germany & ID & $2.4+$ & 50 & $15 \times 15$ & {$[56,57]$} \\
X07MB & SLS & Switzerland & ID & $0.3-8$ & $770(4)$ & $3000 \times 3000$ & {$[58]$} \\
I09 & DLS & UK & ID & $2.1-12+$ & $70(6)$ & $15 \times 30$ & {$[59]$} \\
BL09XU & SPring-8 & Japan & ID & $4.91-100$ & $70(6)$ & $10 \times 1$ & {$[60]$} \\
BL12XU & SPring-8 & Japan & ID & $6-12$ & $100-1000$ & $<40 \times 40$ & {$[61]$} \\
BL15XU & SPring-8 & Japan & ID & $2-36$ & $60(6)$ & $25 \times 30$ & {$[62]$} \\
BL16XU & SPring-8 & Japan & ID & $4.5-40$ & $<250(8)$ & $40 \times 40$ & N/A \\
BL19LXU & SPring-8 & Japan & ID & $7-100+$ & 60 & $6 \times 100$ & {$[26]$} \\
BL22XU & SPring-8 & Japan & ID & $6-70$ & $180(8)$ & $400 \times 500$ & {$[63]$} \\
BL24XU & SPring-8 & Japan & ID & $7-60$ & $350(8)$ & $25 \times 25$ & N/A \\
BL28XU & SPring-8 & Japan & ID & $5.94,7.94,9.94$ & $160(7.94)$ & $400 \times 20$ & {$[64]$} \\
BL29XU & SPring-8 & Japan & ID & $4.9-57$ & $4900(35.45)$ & $500 \times 500$ & {$[65]$} \\
BL36XU & SPring-8 & Japan & ID & $5.2-37.7$ & $160(8)$ & $20 \times 20$ & {$[66]$} \\
BL46XU & SPring-8 & Japan & ID & $4.5-37.5$ & $125(8)$ & $250 \times 20$ & {$[67]$} \\
BL47XU & SPring-8 & Japan & ID & $5.2-37.7$ & $230(8)$ & $30 \times 40$ or 5 51 & {$[60,68]$} \\
PES-BL14 & Indus-2 & India & BM & $3-15$ & $200(4)$ & $4000 \times 1000$ & {$[69]$} \\
SXRMB & CLS & Canada & BM & $1.7-10$ & $200(2)$ & $2000 \times 1000$ & {$[70]$} \\
9.3.1 & ALS & USA & BM & $2.3-6$ & $350(2.3)$ & $300 \times 300$ & N/A \\
5-ID-C & APS & USA & ID & $6-17.5$ & $1500(7)^{\mathrm{a}}$ & $500 \times 1000$ & N/A \\
33-ID-D & APS & USA & ID & $4-40$ & $1500(10)^{\mathrm{b}}$ & $200 \times 100$ & {$[71]$} \\
SST-2 & NSLS-II & USA & ID & $2-8$ & $200(2,6)$ & $<200$ & {$[72]$} \\
\hline
\end{tabular}

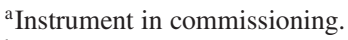

${ }^{\mathrm{b}}$ Improvements underway.

${ }^{\mathrm{c}} \mathrm{We}$ did not receive a response to the survey from BL12XU and have therefore taken the here included information from reference [61].

experiments with a particular focus on resonant excitations [20-24].

A main driver for the development of synchrotron-based HAXPES at the time and even today is the need for high energy resolution [25]. The intrinsic limitations of the laboratory sources available in the late 1990s and early 2000s, including the larger line widths discussed above, limited the applicability of the technique. While the bulk sensitivity of HAXPES is a clear advantage, it can only be exploited fully, if the high energy resolution necessary to resolve core and valence states of interest can be achieved. From 2000 onward development of synchrotron-based beamlines truly accelerated, particularly at SPring-8, Japan [26-31], which received first beam in 1999 and still houses the largest number of beamlines with HAXPES capability in the world (see table 1). The first HAXPES paper from SPring-8 was published in 2003 by Kobayashi and colleagues and used HAXPES at $6 \mathrm{keV}$ to study a $\mathrm{HfO}_{2}$ /interlayer/Si device stack [29]. From 2003, HAXPES instruments were open to users at the European Synchrotron Radiation Facility (ESRF) in Grenoble, France, at beamlines ID16 and ID32, which remained in operation until 2010 (ID16) and 2011 (ID32). These beamlines enabled high intensity and high resolution valence band measurements and standing waves experiments [32-40].
Following the rapid development of HAXPES beamlines worldwide and the parallel establishment and growth of a scientific community a biannual international workshop/conference was established in 2003 with the latest installment taking place in 2019 (a full list of the meetings can be found in the supplementary information (https://stacks.iop. org/JPCM/33/233001/mmedia)). Over the years the growth of the field and its community has been reflected in the growing number of publications available in the area. Figure 1(a) gives an overview of the publication and citation on the topic of HAXPES between the early 2000s and 2020, showing a continuing increase. It should be noted, that over the past few years and with the more widespread use of the technique, an increasing number of publications no longer use the established abbreviations or mention the use of hard $\mathrm{X}$-rays specifically. This results in an underestimation of total publication numbers based on data extracted.

This review on HAXPES, is by no means the first, and follows on from previous review articles on the topic, a special issue on HAXPES in 2013 edited by Wolfgang Drube, as well as the 2016 book edited by Joseph Woicik [41-48]. It provides a comprehensive overview of the available instrumentation across laboratory, synchrotron, and free electron laser sources, as well as the accessible experimental modi of the technique. Throughout the manuscript, fundamental concepts 

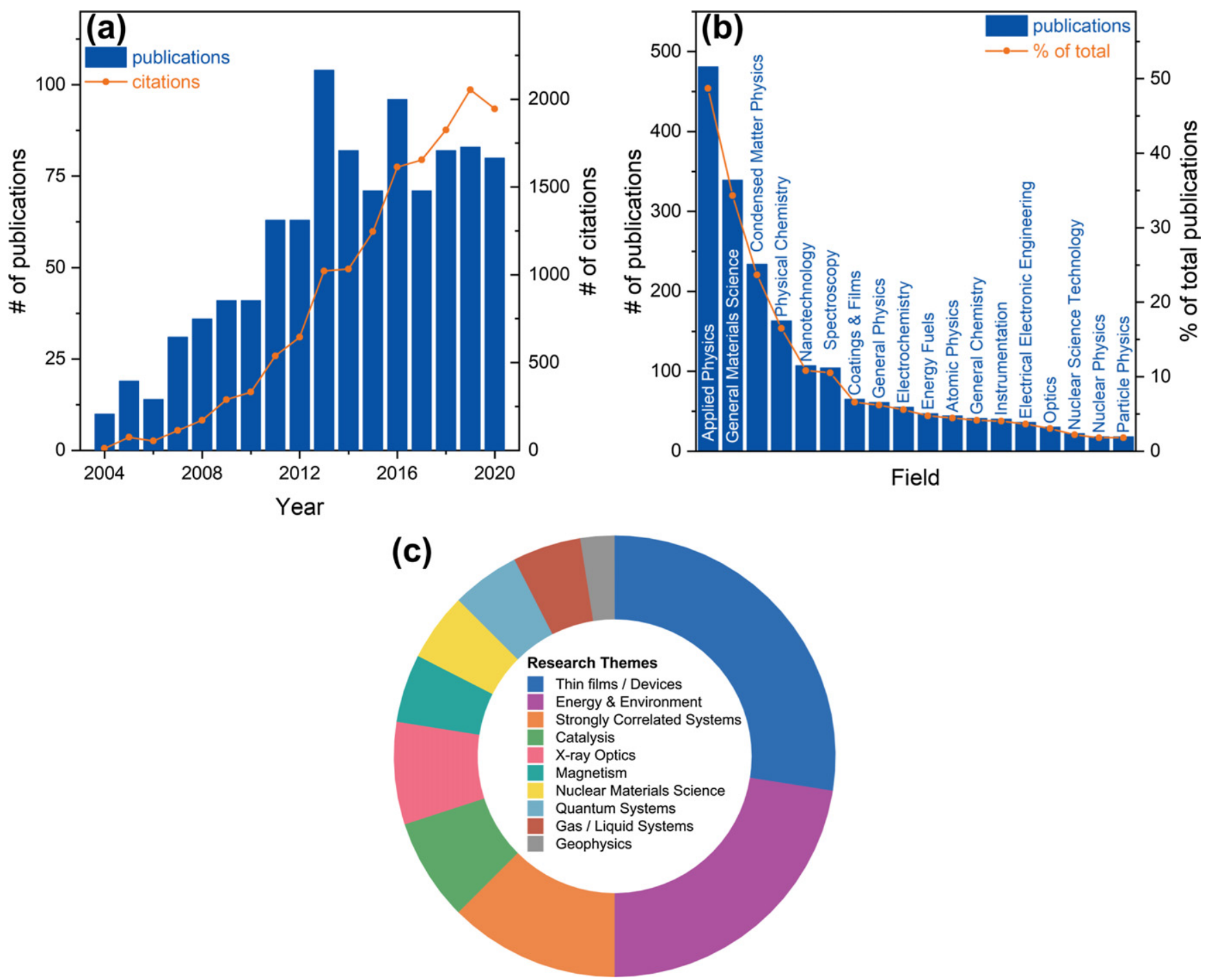

Figure 1. Scientific areas and publication landscape of HAXPES. (a) Number of publications and citations between 2004 and 2020 , (b) number and percentage of publications across different scientific fields between 2004 and 2020, and (c) research areas as identified by the beamline survey. The publication records in (a) and (b) were extracted from Web of Science based on all publications including the search terms 'hard x-ray photoelectron spectroscopy', 'hard x-ray photoemission', 'HAXPES', 'HXPS', 'HX-PES'.

underpinning the technique are introduced where most appropriate, including photoionisation cross sections (section 2.2), accessibility of core levels (section 2.2), information depth and related concepts (section 3.1.1), recoil (section 3.1.1), and angular asymmetry (section 3.2.2). Ultimately, the motivation of the authors has been to provide an unbiased and balanced overview of the state-of-the-art of HAXPES in 2020.

\section{Instrumentation}

This section gives an overview of the three principle sources at which HAXPES experiments can be conducted, including synchrotrons, laboratory sources, and x-ray free electron lasers. Each section provides an up-to-date overview of available experimental stations and their characteristic functionalities. The synchrotron section provides a detailed overview of all currently operational HAXPES beamlines worldwide.

\subsection{Synchrotron-based systems}

As part of this review, we have conducted a survey of the 24 currently operative HAXPES beamlines worldwide in October and November 2020. The survey was circulated and completed by beamline scientists, which are acknowledged below, and key beamline characteristics are presented in table 1. Further information on optics and end stations of each beamline is available in the supplementary information. Beamline $\mathrm{X} 07 \mathrm{MB}$ at the SLS in Switzerland, which is primarily an X-ray absorption beamline, is also included in the overview tables for completeness, although the HAXPES end station is a nonpermanent installation and not in open user operation. Since the early 2000s the number of beamlines available has continued to grow and figure 2(a) gives an overview of the years beamlines came into operation. From the distribution of beamlines worldwide, summarised in figure 2(b), it is clear that SPring- 8 still dominates the field with its sheer number of 

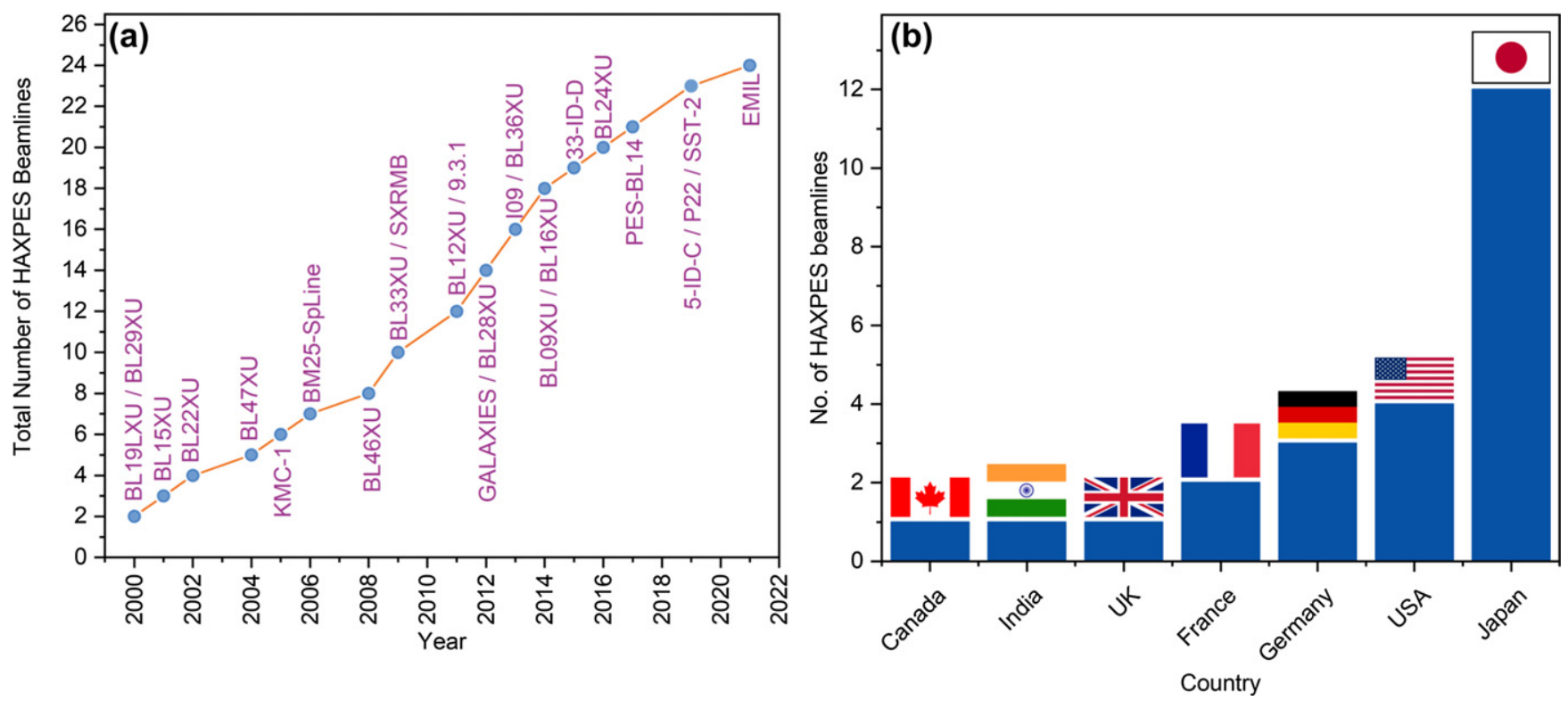

Figure 2. The current worldwide availability of operational HAXPES beamlines. (a) A timeline of the operational starting dates of existing HAXPES beamlines. The data point labels correspond to the beamline identifiers included in table 1. EMIL (BESSY II) is currently undergoing commissioning. Beamline P09 at PETRA III was operational between 2010 and 2018, with the capabilities transferred to beamline P22 in 2019. (b) The number of HAXPES beamlines available within each country.

beamlines available, but strong activities in Europe, the North Americas, and the rest of Asia are continuing to provide ever more improved experimental capabilities and increased user access to the technique.

As is reflected in the publication track record of the technique discussed above and summarised in figures 1(a) and (b), existing beamlines deliver instrument capability to a wide range of scientific communities. As part of our survey we also asked beamline staff to identify the top three research areas their beamline contributes to. Figure 1(c) provides a summative overview of the scientific areas with over half of the responses falling in the energy \& environment as well as thin films \& devices categories. The original answers as given in the survey, and how they were grouped for the preparation of this figure, are included in the supplementary information. The information taken from the survey provides an alternative, complementary view on active scientific areas compared to the information extracted from publication statistics.

The existing HAXPES beamlines do have several aspects in common, but also differ in certain areas, as can be seen from the survey results presented in table 1 and the further tables in the supplementary information. The vast majority of the beamlines is based on insertion devices and only four use bending magnets resulting in variation in the available photon flux with it varying between $10^{11}$ and $10^{13}$ photons/s at the sample position. While a very high photon flux can be an advantage, it should be noted that lower flux density can be an asset when studying materials prone to radiation-induced damage. The accessible x-ray energy ranges mainly differ in the lowest available energy. There is an almost even split between beamlines starting at around $2 \mathrm{keV}$ and beamlines starting at higher energies of $5 \mathrm{keV}$ and above. All beamlines use $\mathrm{Si}(111)$ double crystal monochromators (DCM) and many combine them with channel-cut post-monochromators to achieve high energy resolution at specific photon energies. Large variations in both the best experimental energy resolution and the x-ray spot size at the sample position exist across beamlines and showcase the importance of choosing the most suitable beamline for a given scientific question depending on e.g. sample size, spectra of interest, and intrinsic line width of features. Further important aspects when selecting a beamline for an experiment are the in situ and operando capabilities of the end stations. Many end stations provide a variety of in situ sample preparation facilities, such as sputtering, cleaving and annealing, as well as sample deposition options. The majority of end stations also enables certain operando capabilities, predominantly focused on controlling the temperature of the sample during measurement from cryo-cooling down to as low as $10 \mathrm{~K}$ up to annealing temperatures above $1500 \mathrm{~K}$. Ambient pressure and electrical biasing capabilities, which will be discussed in detail in sections 3.3.2 and 3.3.3, respectively, are gaining popularity due to the ability to conduct dynamic measurements of samples under operation-relevant conditions.

Synchrotron-based facilities are continuing to evolve and new beamlines are being planned and built. After the recent EBS (Extremely Brilliant Source) upgrade of the ESRF, beamline BM25-SpLine is undergoing improvements. BL09XU and BL47XU at SPring-8 will be integrated into BL09XU and will be launched as a new dedicated HAXPES beamline with two endstations in 2021. BL09XU will be temporarily shut down in February 2021, followed by an upgrade to the optics and equipment. After commissioning, the operation is planned to resume in October 2021.

A new HAXPES beamline is currently under commissioning at the Shanghai Synchrotron Radiation Facility (SSRF) and 
is planned to open to users in 2021. BL20U-Energy Materials (E-line) combines soft and hard X-ray techniques aimed at investigating fundamental properties of energy materials and catalysis. The planned beamline specifications include a photon energy range of $130 \mathrm{eV}$ to $18 \mathrm{keV}$ with a flux of $2 \times 10^{12}$ photons/s at $5 \mathrm{keV}$ and $4 \times 10^{12}$ photons/s at $244 \mathrm{eV}$ at $\mathrm{x}$-ray spot sizes of $80 \times 20 \mu \mathrm{m}^{2}$ at $5 \mathrm{keV}$ and $90 \times 10 \mu \mathrm{m}^{2}$ at $244 \mathrm{eV}$, respectively. The beamline will house an ambient pressure PES endstation, which will include a Scienta HIPP-2 analyser and will operate across a photon energy range from 1.5 to $10 \mathrm{keV}$.

The emergence of 4th generation synchrotrons based on multi-bend achromat lattice designs, enabling higher brightness and coherence, promises new possibilities for HAXPES. MAX-IV, the first 4th generation synchrotron had first electrons on the 25th of August 2015 and is now operational. The ESRF upgrade to the Extremely Brilliant Source (EBS) is also complete and first electrons were injected on the 28th of November 2019. Further planned new sources include Sirius in Brasil and the High Energy Photon Source (HEPS) in China, and upgrades are planned for a number of existing synchrotrons, including APS-U, Diamond-II, PETRA IV, and SPring-8-II.

\subsection{Laboratory-based HAXPES}

Over the past decade a new generation of laboratory-based HAXPES systems has emerged, which makes use predominantly of $\mathrm{Cr}(\mathrm{K} \alpha=5.42 \mathrm{keV})$ and $\mathrm{Ga}(\mathrm{K} \alpha=9.25 \mathrm{keV})$ based $\mathrm{X}$-ray sources. The first monochromated $\mathrm{Cr} \mathrm{K} \alpha$-based laboratory HAXPES systems were designed and built in the early 2010s by Keisuke Kobayashi, Hiroshi Daimon, Masaaki Kobata and colleagues [73, 74], who realised the importance of overcoming limitations due to decreasing photoionisation cross sections to enable laboratory-based HAXPES. Their approach to address this challenge was to combine a monochromated, focused, high flux x-ray source with a wideacceptance objective lens and a Scienta R4000 high-energy hemispherical analyser, achieving a competitive energy resolution and speed of acquisition. To date, the majority of HAXPES systems is still located at synchrotron sources due to the mentioned decrease in photoionisation cross sections at higher x-ray energies, which necessitate high x-ray flux to ensure usable signal counting rates. Figure 3 shows the theoretical one-electron cross sections from Scofield for selected orbitals of oxygen and vanadium for energies from 1 to $15 \mathrm{keV}$ extracted and plotted using the Galore software package [75].

Several sets of theoretical cross sections are available in the literature, including by Scofield [76], Yeh and Lindau [77], and most recently from Trzhaskovskaya and Yarzhemsky [78, 79], and these are now available as digitised datasets for ease of use [80-84]. The different cross section datasets clearly illustrate the loss of photoelectrons when moving from the soft into the hard x-ray range. The new, high flux laboratory x-ray sources coupled with highly efficient photoelectron analysers and detectors are enabling us to overcome these limitations and to move HAXPES back in the laboratory environment, where it began using $\mathrm{Cu}$ sources back in the early days of ESCA.

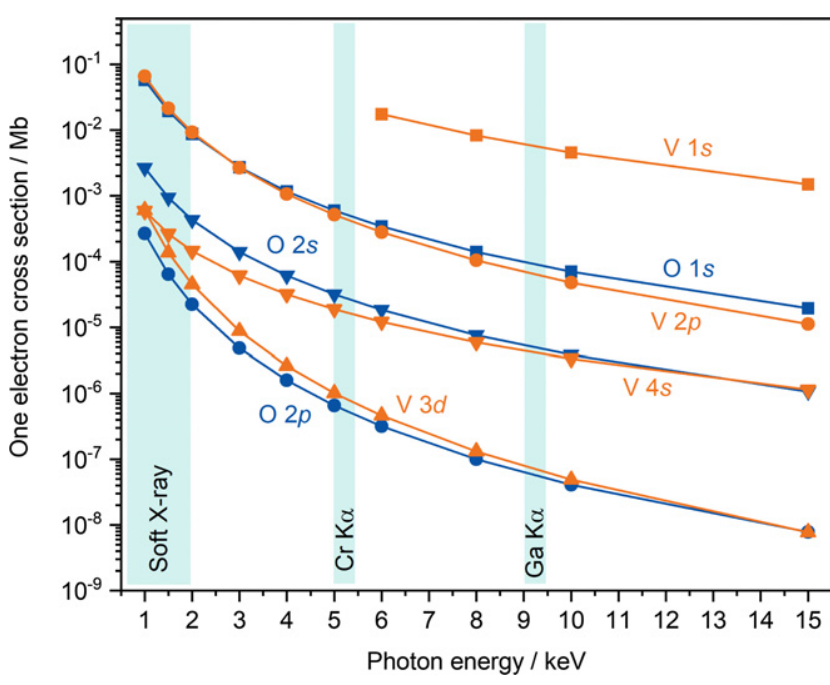

Figure 3. Theoretical one-electron photoionisation cross sections for selected orbitals of oxygen and vanadium across the photon energy range of 1-15 keV. Cross section values taken from [76].

In this section, we give a brief overview of the main commercial and custom-built systems currently available and in operation.

Laboratory-based HAXPES systems have exploited the accessibility of additional, deeper core levels to study depthdependent phenomena in both bulk as well as multilayer systems [85-91]. A general advantage not just of laboratory systems, but any HAXPES experiment, is the ability to access deeper core levels opening up new experimental and analytical strategies [43, 92-95]. Figure 4 gives a schematic overview of core levels of transition metals and lanthanides that become available when moving across the hard x-ray energy range. An example of the usefulness of accessing deeper core levels has been shown in recent work on the satellite structures of transition metal oxides, where using the transition metal $1 s$ rather than the $2 p$ core levels enables a clearer understanding of the spectra features present $[96,97]$. Another example that has been explored with laboratory HAXPES is in complex materials with multiple elements present that often have overlapping core levels in the soft $\mathrm{x}$-ray range, such is the case for $\mathrm{LiNi}_{0.8} \mathrm{Co}_{0.2-y} \mathrm{Al}_{y} \mathrm{O}_{2}$ layered oxide cathodes for batteries [85]. Probing the Al $1 s$ core level instead of the lower energy Al $2 s$ and $2 p$ states excludes spectral contamination from any of the other elements present.

The ability to excite deep core levels consequently also enables the measurement of the associated Auger transitions $[90,100]$. For many industrially relevant materials such as $\mathrm{Al}$, $\mathrm{Si}, \mathrm{Ti}$ and their corresponding oxides and nitrides, the $1 s$ core level is not accessible using monochromated $\mathrm{Al} \mathrm{K} \alpha$ radiation (see figure 4). However, access to these lines enables chemical state analysis using the Auger parameter concept, which is very sensitive to changes in the local chemical environment and often more robust than the observation of core level binding energy shifts alone $[101,102]$. In many instances the Auger parameter is even sensitive to structural changes such as polymorphic phase transitions [103] or the crystallisation of amor- 

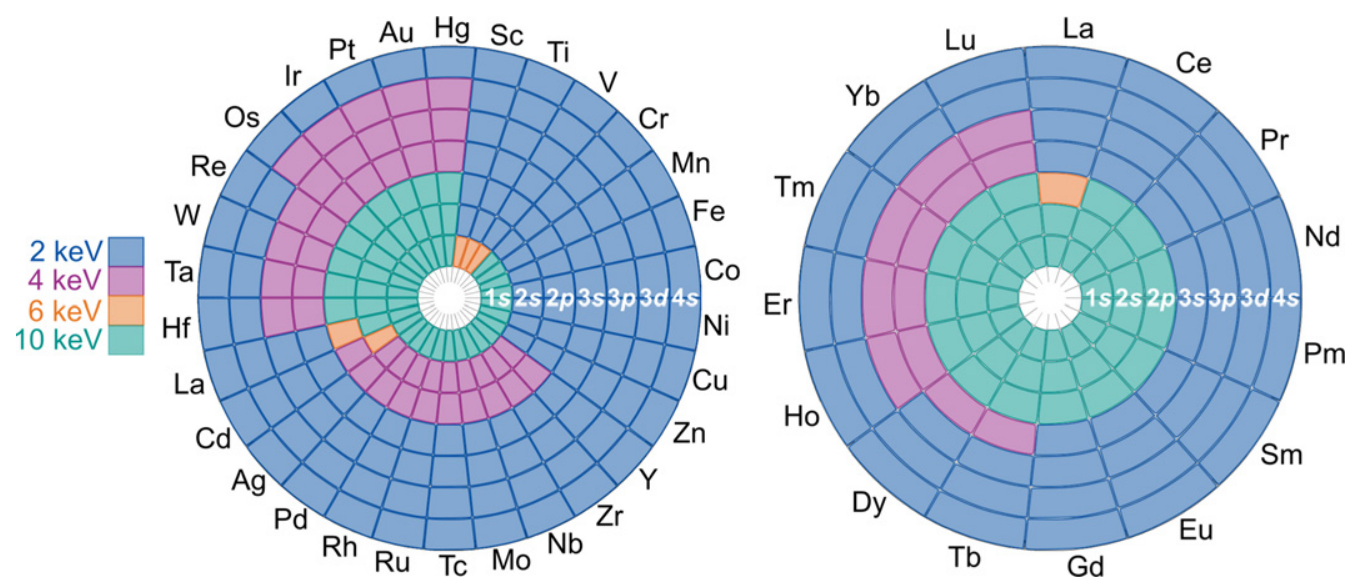

Figure 4. Core levels of the transition metals (L) and lanthanides (R), including the $1 s$ to $4 s$ orbitals. The colors indicate at what minimum excitation energy the core levels can be accessed in photoelectron spectroscopy experiments. All core levels beyond $4 s$ are not included but can all be accessed using excitation energies below $2 \mathrm{keV}$. Information taken from references $[98,99]$.

phous materials [104]. It is worth noting that Auger parameter studies were commonly employed in the 1970s and 1980s and reference values for many materials are reported in literature [102]. This is due to the fact, that older spectrometers often featured non-monochromated $\mathrm{x}$-ray sources, which provided high-energy Bremsstrahlung which could be used to excite deep core levels and the associated Auger lines [105]. Due to the dominance of monochromated soft $\mathrm{x}$-ray sources in today's commercial laboratory XPS systems such measurements are not possible anymore.

Although laboratory HAXPES systems have a lower energy resolution compared to many synchrotron endstations, they have been successfully used to understand the electronic structure of the occupied states (valence bands) of materials $[100,106]$. Meaningful valence band spectra have been collected for established materials, such as rutile $\mathrm{TiO}_{2}$ [100], as well as for novel energy materials, including $\mathrm{Pb}$ and $\mathrm{Sn}$ incorporating $\mathrm{V}_{2} \mathrm{O}_{5}$ nanowires [106], and direct comparison with projected densities of states from density functional theory (DFT) aids the interpretation of specific aspects of their electronic structure. A recent review paper by Razek et al also emphasised the benefits of laboratory HAXPES to accelerate material screening, in particular for energy applications [106]. Finally, laboratory HAXPES systems have been employed to study buried layers and interfaces in device-relevant multilayer samples. Results on Si-based and post-transition metal oxide device multilayers and transistor structures have demonstrated the ability to probe the core level spectra of buried layers and extract information on their chemical state in situ without the need of sputter depth profiling as is necessary in SXPS studies $[87,88,100]$. A recent study on $\mathrm{SiC} / \mathrm{SiO}_{2}$ device multilayers, which are used for power electronic applications, has also demonstrated the opportunity to target specific interface states related to defect states at semiconductor-dielectric interfaces [89]. Several of the studies mentioned here also exemplify the complementarity of laboratory- and synchrotron-based HAXPES systems to enable a comprehensive and efficient exploration of samples of interest $[85,87,89,106]$.
In contrast to synchrotron- or FEL-based HAXPES, the excitation energy in laboratory-based HAXPES systems is fixed to the characteristic $\mathrm{x}$-ray emission of the anode material. This has important implications for the information depth (see general discussion on this topic in section 3.1.1. Depending on the core level which is probed, the binding energy and consequently the kinetic energy of the photoelectrons can vary over a wide range. The largest information depth occurs at the highest kinetic energy and therefore in the valence region, whereas deep core levels occur at lower kinetic energy and thus have smaller information depth. For $\mathrm{TiO}_{2}$, measured with $\mathrm{Cr} \mathrm{K} \alpha$ radiation the difference in information depth $(3 \lambda)$ can reach up $20 \mathrm{~nm}$ [90], and may be even more pronounced for higher excitation energies. One strategy to obtain nearconstant probing depth at one excitation energy it to use the angular dependence of the escape depth of the detected photoelectrons (see detailed discussion in section 3.1.5). This is particularly relevant for the quantification of the depth-dependent composition, as well as depth-dependent chemical state(s) and electronic structure, of heterogeneous materials and thin film systems [107].

Many laboratory-based HAXPES systems are equipped with an additional soft $\mathrm{x}$-ray source, which can provide complementary information on e.g. the surface chemistry and composition, with known sensitivity factor values facilitating quantification efforts. Analysis and quantification of PES data has to take into account not only the photoionisation cross sections, but also the angular asymmetry of photoelectrons (see detailed discussion in section 3.2.2). If the angles between the soft and hard $\mathrm{x}$-ray sources are not the same, then suitable corrections have to be applied. One strategy to circumvent this is implemented in the $\mathrm{Al} / \mathrm{Ag}$ combination of the Kratos AXIS Supra + system, where the same monochromator is used for both hard and soft $\mathrm{x}$-rays, and therefore both $\mathrm{x}$-ray beams are delivered at the sample angle relative to the sample surface. In an ideal setup, the chosen angle is close to the magic angle at about $54^{\circ}$, where the effects of angular anisotropy cancel out. A complementary soft $\mathrm{x}$-ray source is in addition useful in 
calibrating the linearity of the kinetic energy scale of the analyser. The use of dual source designs in laboratory-based HAXPES has additional advantages. The fixed excitation energy in laboratory-based HAXPES can represent a challenge for the calibration of the linearity of the energy scale of the analyser due to a lack of well-defined calibration lines at high binding energies (i.e. low kinetic energies). The combination of soft and hard x-ray sources provides well-defined reference values of the kinetic energies of e.g. Ag and Au core lines across the kinetic energy scale. In single-source HAXPES systems comparatively sharp Auger lines, such as the $\mathrm{Cu} \mathrm{L}_{2,3} \mathrm{M}_{4,5} \mathrm{M}_{4,5}$, can provide alternative reference lines at the lower limit of the kinetic energy scale [90].

2.2.1. PHI Quantes. The first beta HAXPES system from Physical Electronics (PHI Quantes) was shipped in February 2016. The following year, in February 2017, the system was officially introduced to the market. The Quantes is a fully automated photoelectron spectroscopy instrument, specifically designed for spatially resolved chemical analysis and HAXPES, which uses scanned, focused, monochromated $\mathrm{Al} \mathrm{K} \alpha$ and $\mathrm{Cr} \mathrm{K} \alpha$ x-rays. The $\mathrm{Cr} \mathrm{K} \alpha$ source has an excitation energy of $5.4147 \mathrm{keV}$, which enables a probing depth approximately three times greater than $\mathrm{Al} \mathrm{K} \alpha$. The $\mathrm{x}$-ray spot sizes are tuneable from sub-7.5 $\mu \mathrm{m}$ for $\mathrm{Al} \mathrm{K} \alpha$ and $14 \mu \mathrm{m}$ for $\mathrm{Cr} \mathrm{K} \alpha$, respectively, to $200 \mu \mathrm{m}$ or more in diameter for both. When combined with the open-lens analyser design, the detection efficiency can be maximised and x-ray dose for analysis is minimised. For increased count rates both sources feature a high power mode in which the electron beam is scanned on the anode to deconcentrate the thermal load. The same area position on the sample surface can be measured by either $\mathrm{X}$-ray source, which can be focused at the same position of the sample for micro-analysis capabilities using either XPS or HAXPES. The fixed angle between the $\mathrm{Cr}$ and $\mathrm{Al}$ source is $22^{\circ}$ resulting in angles of $45^{\circ}$ and of $49^{\circ}$ with respect to the analyser input lens, respectively. Both angles are quite close to the 'magic angle' which guarantees a reliable element quantification across the depth with both sources [108, 109]. Since February 2020 the system is available with a dual gascluster ion beam (GCIB), which provides surface cleaning and sputter depth profiling capability with minimal damage to surface chemical compositions of inorganic and organic materials.

To date, more than 15 systems have been sold around the world and among them, 13 are installed and operational. In July 2020, a PHI Quantes XPS/HAXPES Scanning Microscope was installed at Empa, Dübendorf, Switzerland. This instrument features an energy resolution of $0.48 \mathrm{eV}$ or $0.85 \mathrm{eV}$ using focused $\mathrm{x}$-ray $\mathrm{Al}$ or $\mathrm{Cr}$ radiation, respectively. It is coupled to a magnetron sputtering chamber and glove box for controlled growth and post-treatment of functional thin films, catalysts and other nanomaterials. Other unique features of the system include a sample holder with four-contact heating/cooling stage to perform electrical measurements while acquiring photoelectron spectra, as well as an additional smaller glove box at the intro-chamber to further facilitate measurements on sensitive samples.
2.2.2. Scienta Omicron HAXPES lab. In 2018 Scienta Omicron brought their first integrated HAXPES Lab system onto the market. It uses an Excillum MetalJet-D2+ $70 \mathrm{kV}$ $\mathrm{x}$-ray source, which is based on a Ga metal-jet anode, and together with a Si monochromator delivers $\mathrm{Ga} \mathrm{K} \alpha$ x-rays at an energy of $9.25 \mathrm{keV}$ [110-112]. The liquid Ga jet enables the use of a small spot, high intensity electron beam to generate electrons without the anode degeneration often suffered by solid anodes at high power loads. This in turn gives a high photon flux, of $6.8 \pm 0.2 \times 10^{8}$ photons/s at the sample position, necessary for HAXPES experiments in the laboratory. The Excillum X-ray source can operate across a range of power settings, from 50 to $250 \mathrm{~W}$, giving tunability of the $\mathrm{x}$-ray flux produced while keeping the energy resolution constant. The $\mathrm{X}$-ray spot size is $30 \times 45 \mu \mathrm{m}^{2}$ when it enters the analysis chamber. The rotational movement of the four-axis manipulator in the analysis chamber allows the angle between the incoming X-rays and the sample surface to be finely controlled, including the use of reproducible, grazing incidence geometry. The high energy Scienta Omicron EW4000 hemispherical electron energy analyser (HEA) with its large acceptance angle of $60^{\circ}$ coupled with a $2 \mathrm{D}$-detector setup consisting of a multi-channel plate (MCP), phosphor screen, and a CMOS camera enables the collection of both core and valence spectra in reasonable time frames and with an energy resolution of below $500 \mathrm{meV}$. Various additional options can be added to the Scienta Omicron HAXPES Lab system, including complementary soft X-ray $\mathrm{Al} \mathrm{K} \alpha$ sources, and sputter ion and gas cluster ion beam sources. Furthermore, the design allows connection to other modules, such as preparation chambers, glove boxes, or other complementary analysis techniques. A detailed description of the system can be found in the work by Regoutz et al [100]. As discussed above, relative sensitivity factors (RSFs) are needed for quantitative measurements. Efforts across all laboratory systems are ongoing and the first step toward reliable RSFs for the Scienta Omicron HAXPES Lab has been recently taken by the team at the University of Manchester [113]. A library of all core levels accessible for elements up to $Z=99$ using the Ga K $\alpha$ source up to a binding energy of $9 \mathrm{keV}$ has been computed with ratification of the values ongoing.

To date, six HAXPES Lab systems have been installed and are operational in Japan (at Meiji University, Hyogo Prefecture and MST), the UK (at the University of Manchester), Belgium (at IMEC), and the USA (at Binghamton University). A number of these systems, including the one at Hyogo Prefecture, combine the Ga X-ray source with an $\mathrm{Al} \mathrm{K} \alpha$ x-ray source to benefit from the comparison of surface and bulk sensitive SXPS and HAXPES. The existing HAXPES Lab systems have already delivered a number of useful insights into a range of scientific areas, reflecting the strength of laboratory-based HAXPES in general to capitalise on many advantages of the technique previously only accessible at synchrotron sources (see introduction to this section and references therein).

2.2.3. SPECS systems. Four SPECS-based HAXPES systems are in operation in Germany, the UK, and South Korea, which all use $\mathrm{Cr} \mathrm{K} \alpha \mathrm{x}$-ray sources. The $\mathrm{x}$-ray source uses 
an anode voltage of up to $30 \mathrm{kV}$ and a maximum power of $150 \mathrm{~W}$. It can deliver a spot size between 100 and $1000 \mu \mathrm{m}$. The monochromator is based on a Ge(443) single crystal in a Rowland circle with a diamater of $730 \mathrm{~mm}$. The majority of these systems are ambient pressure HAXPES (APHAXPES) setups, including a liquid AP-HAXPES setup at the Fritz Haber Institute in Germany and a standard APHAXPES system at the University of Manchester in the UK combining $\mathrm{Al}$ and $\mathrm{Cr} \mathrm{K} \alpha$ sources. A special case of a laboratory ambient pressure HAXPES system is beamline 8A at the Pohang Accelerator Laboratory, South Korea's synchrotron. While the end station uses an undulator-based beamline for measurement in the soft $\mathrm{x}$-ray regime, it uses an offline $\mathrm{Cr}$ $\mathrm{K} \alpha$ source installed on the same end station to enable bulk measurements in parallel to surface sensitive ambient pressure experiments [114].

2.2.4. Kratos systems. Kratos started to build systems with hard x-ray sources including $\mathrm{Ag}$ and $\mathrm{Zr}$ in the 1980s. In the newest Kratos spectrometer generation a dual monochromated Al $\mathrm{K} \alpha / \mathrm{Ag} \mathrm{L} \alpha \mathrm{x}$-ray source is implemented in their AXIS Supra $^{+}$system. The same monochromator crystal is used for both, as the photon energy of $\mathrm{Ag} \mathrm{L} \alpha(h \nu=2.984 \mathrm{keV})$ is approximately double that of $\mathrm{Al} \mathrm{K} \alpha$ and the second order diffraction of the monochromator crystal used for Al K $\alpha$ can be used to also deliver monochromated Ag L $\alpha$ x-rays. This is not only a very efficient way of delivering two monochromated $\mathrm{x}$-ray beams of differing energy, but it also enables two beams at the same angle compared to the overall photoemission geometry. A useful practical feature of the Kratos system is the full automation of switching between the two excitation sources allowing combinatorial soft and hard x-ray measurements.

Kratos started selling the $\mathrm{Ag} \operatorname{L} \alpha$ as an optional excitation source with the AXIS Ultra/AXIS Nova instruments in 2000 and 60 instruments of this earlier instrument generation were sold. In addition, 90 dual Al/Ag monochromators have been sold for the current AXIS Supra/AXIS Supra ${ }^{+}$instruments, making $\mathrm{Ag} \operatorname{L} \alpha$ the most widely available laboratory hard $\mathrm{x}$-ray source. The $\mathrm{x}$-ray illuminated area is $\approx 2 \times 1 \mathrm{~mm}$ and an aperture in the lens column is used to define the analysis area. Importantly, as the analysis area is defined by transfer lenses and not the $\mathrm{x}$-ray spot location, the acquired spectra originate from the same location on the sample irrespective of which source is selected. The large area analysis setting gives an area of $700 \times 300 \mu \mathrm{m}$ and it is possible to use selected area spectroscopy modes to access smaller analysis areas of $110 \mu \mathrm{m}, 55 \mu \mathrm{m}, 27 \mu \mathrm{m}$, or $15 \mu \mathrm{m}$ in diameter. Using the large area analysis mode a full width at half maximum (FHWM) of below $0.9 \mathrm{eV}$ on the $\mathrm{Ag} 3 d_{5 / 2}$ core level can be achieved with good signal to noise (a typical count rate being $6 \mathrm{kcps}$ ).

One challenge faced by many laboratory-based HAXPES instruments is the lack of reliable RSFs and knowledge of the transmission function at higher binding energies, which usually requires in-depth reference measurements. Kratos addressed this exact challenge through a systematic measurement series resulting in a reproducible transmission function and the Cant RSFs for Ag L $\alpha$ excited spectra [115]. The, in the context of hard x-ray sources, low energy of the Ag source limits the exploration of deeper core levels. However, this is partially mitigated by the comparatively good photoelectron cross sections of many of the lighter elements. The $\mathrm{Ag} \operatorname{L} \alpha$ has found successful application in a number of areas [116-119].

2.2.5. Custom systems. A number of custom built systems are also in operation often combining $\mathrm{x}$-ray sources and analysers from different suppliers.

Kochi University of Technology, Japan, has a double x-ray source laboratory HAXPES system, which combines monochromated $\mathrm{Cr} \mathrm{K} \alpha$ and $\mathrm{Al} \mathrm{K} \alpha$ x-ray sources from ULVAC-PHI with a Scienta Omicron EW4000 analyser. It also houses a dual beam charge neutralisation setup using a low energy electron beam and ion beam (ULVAC-PHI) and a connected magnetron sputtering deposition system (Chemitronics) for in situ characterisation [120-122].

One of the most recent additions to the family of $\mathrm{Cr} \mathrm{K} \alpha$-based HAXPES systems has been constructed and commissioned at Temple University (USA). The heart of the system is a custom-built high-flux monochromated $5.4 \mathrm{keV}$ hard x-ray microfocus source, consisting of a $30 \mathrm{kV}$ focused electron gun (Kimball Physics), a water-cooled thin-film $\mathrm{Cr}$ anode, and a large bent-crystal Ge(411) monochromator in a $730 \mathrm{~mm}$ Rowland circle geometry. The $\mathrm{x}$-ray spot size is variable from 150 to $500 \mu \mathrm{m}$. The standard working energy resolution of the source is $<430 \mathrm{meV}$ and a stable photon flux of $5.9 \times 10^{9}$ photons/s is achieved at standard operating conditions with $2 \mathrm{~mA}$ emission current. The above-mentioned $\mathrm{x}$-ray source has been integrated into a versatile system allowing for the full range of angle-resolved investigations of solids and interfaces, including both core-level and valence band spectroscopies. For such studies, the system has been equipped with a wide-acceptance-angle electrostatic photoelectron analyser Scienta Omicron EW4000, which enables 'one-shot' depth profiling of samples by utilizing different angular segments of the detector for angle-resolved HAXPES. To complement this capability, a custom-built six-axis $(x, y, z$, polar $\theta$, tilt $\beta$, and azimuth $\phi$ ) sample manipulator facilitates a temperature-range from 5 to $380 \mathrm{~K}$. Operando capabilities have also been implemented for measurements on active devices. This system has been recently utilised to investigate changes in the valenceband density of states due to strain-induced anion ordering in perovskite oxyfluoride epitaxial thin films [123].

\subsection{X-ray free electron lasers}

All storage rings and free-electron lasers (FELs) are pulsed sources of synchrotron radiation delivering a highly brilliant beam in the form of $\approx 10-100$ ps and $\approx 10-100$ fs pulses, respectively. In principle, these short-pulsed beams can readily be used as a probe in stroboscopic pump-probe experiments, opening the way to direct dynamical measurements from the picosecond-to-nanosecond timescales of 'slow' phase transitions and charge-carrier relaxation down to the sub-picosecond regime of ultrafast fundamental electronic, magnetic, chemi$\mathrm{cal}$, and structural processes in condensed matter. In practice, however, there is the significant experimental challenge that the data acquisition rate is restricted by the repetition rate 
Table 2. Selected recent pico- and femtosecond time-resolved photoemission spectroscopy experiments at storage rings and free-electron lasers. The listed experimental parameters are the probe photon energy at which experiments were conducted $(h \nu)$, the effective pulse repetition rate $\left(f_{\text {rep }}\right)$, the effective energy $(\Delta E)$, and the time $(\Delta t)$ resolution. For storage ring-based (FEL-based) experiments, $f_{\text {rep }}$ is determined by the repetition rate of the pump laser (FEL probe) pulses.

\begin{tabular}{lllllllc}
\hline Type & Beamline & Facility & $h \nu / \mathrm{keV}$ & $f_{\text {rep }} / \mathrm{kHz}$ & $\Delta E / \mathrm{eV}$ & $\Delta t / \mathrm{ps}$ & Reference \\
\hline Storage ring-based & TEMPO & SOLEIL & 0.12 & 141 & 0.012 & 50 & {$[125]$} \\
& BL07LSU & SPring-8 & 0.385 & 208 & 0.7 & 50 & {$[126]$} \\
& 11.0 .2 & ALS & 0.95 & 127 & & 60 & {$[127]$} \\
FEL-based & BL19LXU & SPring-8 & 7.94 & 1 & $<0.5$ & 50 & {$[128]$} \\
& PG2 & FLASH & 0.11 & 5 & 0.13 & 0.16 & {$[129]$} \\
& SXR & LCLS & 0.85 & 0.12 & $\approx 1.4$ & 0.28 & {$[130]$} \\
& BL3 & SACLA & 8 & 0.03 & $\approx 1.25$ & $\approx 0.25$ & {$[131]$} \\
\hline
\end{tabular}

of the source and the efficiency of the detection. For timeresolved pump-probe photoemission measurements in particular, both factors are limited. First, the (pump) pulse repetition rate typically cannot be higher than $1 \mathrm{MHz}$ to allow for sufficient sample recovery time between consecutive pump pulses. Second, the number of the photoemitted electrons has to stay below about $10^{3}$ to $10^{4}$ per pulse due to vacuum space-charge effects [124], while the number of detected electrons per pulse is practically often only about 1 . To date, as we have not yet reached the $1 \mathrm{MHz}$ limit, the major bottleneck of timeresolved photoelectron spectroscopy (PES) toward the HAXPES regime is the lack of short-pulsed high-repetition-rate $\left(1 \mathrm{kHz}<f_{\text {rep }} \leqslant 1 \mathrm{MHz}\right.$ ) sources in the soft to hard $\mathrm{x}$-ray range. Table 2 provides an overview of recent, exemplary picoand femtosecond time-resolved optical pump/x-ray probe PES experiments performed on condensed matter systems at photon energies larger than $100 \mathrm{eV}$.

Picosecond time-resolved soft x-ray PES can be performed at storage rings in special operation modes, e.g., at the beamlines TEMPO of SOLEIL [125, 132], BL07LSU of SPring-8 [126, 133], and 11.0.2 of the ALS [127, 134]. The effective time resolution is typically $50-60 \mathrm{ps}$, given by the duration of the synchrotron radiation pulses, and the (pump laser) repetition rates are in the range of $100-300 \mathrm{kHz}$. To our knowledge, the only picosecond time-resolved HAXPES experiment so far has been done at beamline BL19LXU of SPring-8, with a time resolution of $50 \mathrm{ps}$ at a probe photon energy of about $8 \mathrm{keV}$ and a repetition rate of $1 \mathrm{kHz}$ [128].

At present, the only facility where femtosecond timeresolved core-level PES measurements are routinely done is beamline PG2 of the extreme ultraviolet/soft x-ray FEL FLASH [135-137]. The high effective (probe pulse) repetition rate of $5 \mathrm{kHz}$ allows for ultrafast core-level spectroscopy with combined high spectral and temporal resolution of $130 \mathrm{meV}$ and $150 \mathrm{fs}$, respectively [129, 137]. However, the practically available probe photon energy reaches into the low-energy part of the soft x-ray regime only (to about $700 \mathrm{eV}$ in the third harmonic), and the repetition rate is still two orders of magnitude below the $1 \mathrm{MHz}$ limit. In pioneering experiments at the hard x-ray FELs LCLS [130] and SACLA $[131,138,139]$, the probe photon energy has been pushed to $850 \mathrm{eV}$ and ultimately to $8 \mathrm{keV}$, i.e., deep into the HAXPES regime. Despite the extremely low repetition rates of the hard $\mathrm{x}$-ray FEL radiation sources $(120 \mathrm{~Hz}$ and $30 \mathrm{~Hz}$, respectively), significant time-dependent core-level data reflecting interfacial charge transfer and charge carrier recombination dynamics could be obtained with sub-300 fs time resolution [130, 131].

In conclusion, as of November 2020, only three 'real' sub100 ps time-resolved HAXPES experiments have been done: one at the storage ring SPring-8 [128] and two at the SACLA FEL $[131,139]$. Yet, supplemented by numerical space-charge simulations [140], these experiments have demonstrated that FEL-based femtosecond time-resolved HAXPES is a fully viable technique that becomes practical at the highest possible average photon flux and electron detection efficiency. With the parallel development of the time-of-flight (ToF) momentum microscope (see section 3.1.3) and fast multi-hit electron detectors, a highly efficient 3D electron detection system is now available [141, 142]. In addition, with the advent of the high-repetition-rate hard x-ray FELs European XFEL $(27 \mathrm{kHz})$ and LCLS-II (1 MHz), the future looks also bright on the source side.

\section{Exploring multiple dimensions with HAXPES-available experimental modi}

The following sections present 13 core areas of HAXPES and recent developments and advancements in these areas. To aid navigation and structuring of the large number of distinct areas, the topics are divided into three subgroups depending on the detection scheme, the excitation scheme, and the sample or sample environments explored. The first section covers different detection schemes discriminating in energy, angle, position and time, which covers energy-dependent HAXPES, angleresolved HAXPES (HARPES), momentum microscopy, hard $\mathrm{X}$-ray photoelectron diffraction (hXPD), angular-dependent HAXPES (AR-HAXPES), hard x-ray photoemission electron microscopy (HAXPEEM), spin-resolved HAXPES, and timeresolved HAXPES (trHAXPES). The second section includes different excitation schemes such as X-ray standing waves (XSW) and polarisation-dependent HAXPES. The final part covers varying samples and sample environments, including gas phase HAXPES, ambient pressure HAXPES (APHAXPES), and operando HAXPES of devices. 


\subsection{Detection schemes}

3.1.1. Energy-dependent HAXPES. Many HAXPES experiment start with the measurement of spectra at a single hard $\mathrm{x}$-ray excitation energy in fixed geometry. This can deliver useful information on the bulk of a material, as discussed in the context of laboratory-based HAXPES (see section 2.2). The energy-dependence of both photoionisation cross sections and probing depth can been exploited further by tuning the x-ray energy and collecting spectra at a number of energies. In the case of core level spectra the main interest is to follow changes in chemical states with probing depth, while for valence spectra the motivation is often to distinguish contributions with varying orbital character. Although multi-source laboratory systems exist, as discussed in section 2.2, the tunability of synchrotron sources results in most energy-dependent experiments being conducted at synchrotron beamlines. A multitude of studies has been conducted in this fashion over the years and it is impossible to cover them all, however, a selection of specific, exemplary studies is discussed here to illustrate the applicability of the energy-dependent HAXPES approach.

Before moving on to individual examples, it is useful to recall the concept of information depth in HAXPES at this point, including available models and specific considerations when using hard x-rays. A number of comprehensive discussions of the concepts covered here are available in the literature, and the following is only a brief summary [47, 143, 144]. In HAXPES experiments the $\mathrm{x}$-ray absorption length is much greater than the inelastic mean free path (IMFP) of the excited photoelectrons, and therefore the information depth is determined only by the IMFP. Several approaches for the estimation of IMFPs have been developed with the most popular being those of Seah and Dench, and Tanuma, Powell and Penn (the TPP-2M method) [145-148]. Inelastic background modeling has been explored to detect deeply buried layers beyond the elastic limit [113, 149].

While the IMFP finds wide application to estimate the probing depth in HAXPES, and generally in PES, it does not include effects from elastic scattering, which can play a significant role in determining the information depth, depending on excitation energy and atomic number. When elastic scattering effects are important, the effective attenuation length (EAL) is used instead of the IMFP, which gives smaller information depths due to losses from elastic scattering [34, 150-153]. It is important to note that theoretical models generally overestimate the probing depth of HAXPES [154, 155]. Solokha et al studied the difference between theoretical and experimental EALs in silicon across kinetic energies from 1.5 to $8 \mathrm{keV}$ at beamline I09 at Diamond Light Source [154]. The experimentally determined EALs are significantly smaller than the predicted EALs and IMFPs. One particular observation is that at higher energies above $15 Z^{2} \mathrm{eV}$, where $Z$ is the atomic number of the element, a better description of the elastic scattering cross section is necessary. Some additional considerations have to be taken into account when determining the information depth in liquids rather than solids. This is becoming important in HAXPES as ambient pressure setups enable experiments in liquids and at solid/liquid interfaces (as will be discussed in detail in section 3.3.2) [156, 157].

For both cases with and without elastic scattering being taken into account, the depth distribution function (DDF) is defined as the probability that a photoelectron leaving the surface originated from a given depth measured normally from the surface, and a number of approaches exist to calculate it [158]. DDFs can be particularly illustrative when trying to visualise the information depth and distribution in multilayered samples [89]. Finally, a useful definition in this context is that of information depth (ID) itself. It is generally defined as the depth normal to the sample surface from which useful information can be obtained. IDs are given as the percentage of detected signal across the total depth and common IDs such as $\mathrm{ID}_{90}, \mathrm{ID}_{95}$, and $\mathrm{ID}_{99}$ represent the depths from which 90 , 95, and $99 \%$ of the total signal originate from, respectively.

Many HAXPES studies involve the tuning of the excitation energy to collect core level spectra carrying varying depth information to create non-destructive depth profiles. In addition, such studies can be used to experimentally determine IMFPs for specific systems. Ouardi et al combined these two motivations and performed a systematic exploration of probing depth in HAXPES on the Heusler compound $\mathrm{Co}_{2} \mathrm{MnSi}$, using hard $\mathrm{x}$-ray energies between 1 and $8 \mathrm{keV}$ combined with specifically designed test samples with varying layer thicknesses [159]. To clearly demonstrate the depth dependence in HAXPES they employed a partially oxidised Ta layer underneath a $1 \mathrm{~nm}$ thick Pt layer. With higher excitation energy the oxide peak in the Ta $4 d$ core level reduces, reaching equal intensity with the metal peak at $4 \mathrm{keV}$, and completely vanishing at $7 \mathrm{keV}$. From these experiments and knowledge of the IMFPs the authors could conclude that the top $3 \mathrm{~nm}$ of the Ta layer were oxidised. Such experiments are very useful, to determine varying oxide thicknesses on the surface of materials. Furthermore, they find application when trying to design capping layers to protect oxygen or moisture-sensitive layers from air exposure, a practice common in PES. By tuning the energy to probe through capping layers of different thicknesses the oxidation of the underlying layer of interest can be studied. Beyond the demonstration of a non-destructive depth profile of the $\mathrm{Ta} / \mathrm{Pt}$ system, Ouardi et al went on to experimentally determine the IMFP of $\mathrm{Co}_{2} \mathrm{MnSi}$, by using $\mathrm{Ta}(40 \mathrm{~nm}) / \mathrm{Co}_{2} \mathrm{MnSi}$ $(x) / \mathrm{Pt}(1 \mathrm{~nm})$ heterostructures with varying thickness $x$ of the $\mathrm{Co}_{2} \mathrm{MnSi}$ interlayer. By recording the intensities of the Pt and Ta core level spectra and comparing area ratios they extracted experimental IMFP values between 2 and $7 \mathrm{keV}$, which as expected were lower than those calculated from theory.

Energy-dependent HAXPES can be approached from two different perspective. One can either vary the excitation energy, as in the previous example, or one can vary the core level measured for a specific element to build up non-destructive depth profiles. The latter is enabled by the increased number of deeper core levels accessible with HAXPES compared to SXPS, as discussed in section 2.2, coupled with the difference in depth information depending on the kinetic energy of the photoelectrons coming from different core levels. Instead of 
collecting only one core level for an element at many different excitation energies, multiple core levels of the same element with significantly different energies are collected at the one (or more) excitation energies. Such an approach has been applied successfully in Si-containing systems, including silicon nanoparticle anodes in lithium ion batteries and $\mathrm{SiC}_{\mathrm{SiO}}$ structures used in power electronic devices $[89,160]$. The silicon $1 s$ core level together with either the $2 s$ and $2 p$ core levels, which have elemental reference binding energies of 1839,150 and $100 \mathrm{eV}$, respectively, can be effectively combined to provide a detailed depth profile and aid tuning of the depth information to layers or interfaces of specific interest. One point of note is that while most energy-dependent studies are performed at synchrotron sources due to the tunability of such sources, this strategy can also be implemented using laboratory sources.

HAXPES is often used to solve arguments about whether certain spectral features may be assigned to surface states of some description or whether they are intrinsic to the photoemission in a material. An early study by Payne et al used HAXPES at 6 and $7.7 \mathrm{keV}$ in comparison with Al K $\alpha$ SXPS to understand the appearance of two components in the $\mathrm{Pb} 4 f$ core spectra of $\beta-\mathrm{PbO}_{2}$ [161]. They could show that the presence of a narrower, low binding energy component with predominant Gaussian character together with a broader, high binding energy component with predominant Lorentzian character could be explained by the presence of screened and unscreened states, respectively, rather than by an ill-defined surface state.

Beyond the information of elemental and chemical distributions with depth, energy-dependent core level HAXPES has also been used to quantify changes in electronic and magnetic behavior with depth. Mukherjee et al studied the distribution of charge carriers in prototypical $\mathrm{LaAlO}_{3} / \mathrm{SrTiO}_{3}$ oxide heterostructures [162]. This system famously exhibits a highly mobile, two-dimensional electron gas (2DEG) at the interface of two insulating, diamagnetic oxides. Using excitation energies between 2 and $8 \mathrm{keV}$, La $3 d$ and $\mathrm{Sr} 3 d$ core level spectra were recorded to tune the excitation energy to the interface. Then, Ti $2 p$ spectra could be used to determine the charge carrier distributions in the system. The authors found that two distinct distributions could be observed with one distributed homogeneously throughout the bulk $\mathrm{SrTiO}_{3}$ and another specific to the interface with a with of $\approx 1 \mathrm{~nm}$. Pincelli et al used excitation energies from 0.8 in the soft $\mathrm{x}$-ray regime to $5.94 \mathrm{keV}$ in the hard $\mathrm{x}$-ray regime to determine the critical thickness of electron hybridisation in the spintronic materials $\mathrm{La}_{1-x} \mathrm{Sr}_{x} \mathrm{MnO}_{3}$ and (Ga, Mn)As [163]. They used the evolution of the ratio between the peak areas of the main photoionisation peak and a well screened peak as a function of the respective IMFPs to show that (Ga, Mn)As and $\mathrm{La}_{1-x} \mathrm{Sr}_{x} \mathrm{MnO}_{3}$ have intrinsic limits of 3 and 10 unit cells from the surface before bulk properties are restored.

An important fundamental process of photoionisation, that is often overlooked in energy-dependent HAXPES, is interchannel coupling. While is broadly assumed that photoionisation cross sections are dominated by single-particle behavior, particularly for deep inner shells and hard x-ray photon energies, Drube et al showed that this approximation in fact breaks down due to the presence of electron correlations and interchannel coupling [164]. This process becomes relevant in the interpretation and analysis of core level spectra, when core level intensities are evaluated quantitatively and the photon excitation energy is 'near' a deep core level threshold. Then, the absolute and relative intensities of shallower core levels may change, potentially leading to misinterpretation. Depending on the material studied and the photon energy range across which experiments are performed, this effect can significantly alter the observed core levels, as was shown on the examples of the Ag and In by Drube et al, where interchannel coupling of the $3 d$ photoionisation channels with the $2 p$ channels occurs.

An in practice tremendously useful aspect of being able to tune the excitation energy is that Auger lines occur at the same kinetic energy independent of the excitation energy and can therefore be moved relative to core levels of interest to avoid overlap. This is of particular interest in complex multi-element materials, such as high entropy alloys and complex ceramics for solid oxide fuel cells. An example of using energy-dependent PES at beamline KMC-1 at BESSY II applied to a multicomponent alloy $(\mathrm{CrMnFeCoNi})$ is shown in figure 5 [165]. When this material is studied using standard $\mathrm{Al} \mathrm{K} \alpha$ laboratory sources, the overlap in the spectra of Auger electrons and metal $2 p$ photoelectrons impairs the determination of chemical shifts and relative atomic concentrations vital to gain an understanding of the material. By tuning the excitation energy at the synchrotron to $2 \mathrm{keV}$, the same binding energy region lacks said overlaps due to the change in position of the Auger lines and a complete analysis and interpretation of the transition metal core level spectra is possible. For applications, such as the study of the composition of high entropy alloys, the opportunity to shift away the Auger spectra removes a significant obstacle to a detailed spectroscopic analysis of the multicomponent alloys of transition metals.

Hard X-rays also open the realm of $1 s$ resonances for the second row elements, starting with $\mathrm{P} 1 s$, which has a binding energy of $2.145 \mathrm{keV}$. Using tunable hard $\mathrm{x}$-rays the electron emission from a decaying core-excited state can be studied in kinetic energy regions, where the normal Auger spectrum manifests, if the photon energy is (far) above the core ionisation threshold. As an example, resonant core excitation of $\mathrm{S} 1 s$ into unoccupied bound states of sulfur atoms in various environments and the decays of those core excited states observed in the S KLL Auger kinetic energy region will be discussed here. The electron spectrum in the Auger region is considered static for excitations above the ionisation threshold. If the photon energy is stepped over e.g. the K-edge absorption resonance of sulfur, a significant variation of intensity in the X-ray absorption spectrum is noticable. If the electron kinetic energy spectrum in the S KLL region is recorded at the same time, deviations from the normal Auger spectrum will be readily observed. Parts of the spectrum will remain static in kinetic energy and eventually transition to building up the normal Auger spectrum, other parts are static in binding energy - hence dispersing on the kinetic energy scale. The 


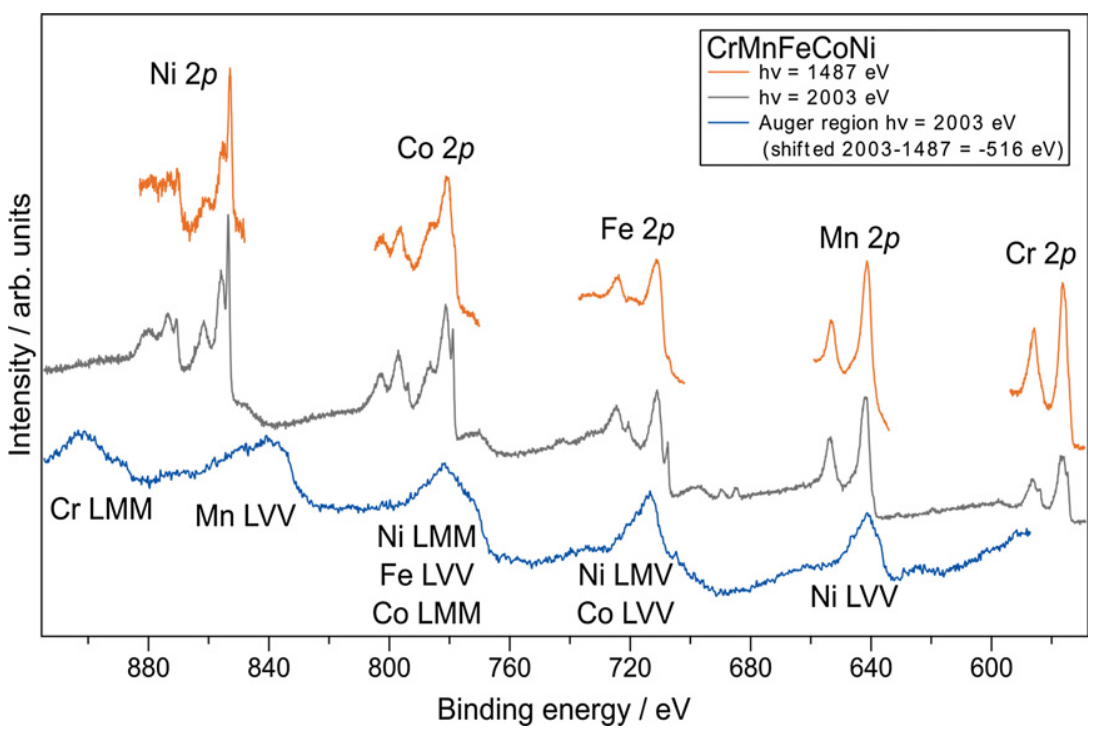

Figure 5. Transition metal $2 p$ core level and Auger spectra of a CrMnFeCoNi multicomponent alloy collected using a laboratory-based $\mathrm{Al}$ $\mathrm{K} \alpha$ source and $2 \mathrm{keV}$-rays at beamline KMC-1 at BESSY II. The Auger region was shifted by $516 \mathrm{eV}$ to aid comparison and illustrate overlap.

latter parts are intense close to resonance and vanish above the ionisation threshold. By recording an electron spectrum for photon energy steps over the resonance a map of the decay channels present in the system can be obtained. This forms the basis for resonant Auger spectroscopy.

Core hole clock spectroscopy (CHCS) utilises the life time of the core hole $\left(\tau_{\mathrm{ct}}\right)$ as an internal time-reference and uses the relative intensity between the channels static in kinetic energy, denoted Auger channels since the final state is Auger-like even below the ionisation threshold owing to that the core-excited electron have tunneled away, and those of dispersing states, denoted Raman channels (see Karis et al for details) [166]. If a resonant Auger spectrum is collected at a particular photon energy, the ratio between the intensities of the Raman $\left(I_{R}\right)$ and Auger $\left(I_{\mathrm{A}}\right)$ channels, $I_{\mathrm{R}} / I_{\mathrm{A}}$, multiplied with the core hole lifetime, e.g. that of S $1 s\left(\tau_{1 s}\right)$, reveals the time at which the excited electrons have moved away from the site of core-excitation. Using the core-hole clock method electron dynamics in the fs to tens of as regimes can be studied with the chemical specificity that core excitations provide $[167,168]$.

Johansson et al, have used the CHCS methodology to study fs and as electron-transfer dynamics in an organic heterojunction [169]. For core excitations close to the K-edge a significant variation between charge transfer times is observed depending on the PCPDTBT/PCBM weight ratio in the active layer, with the time reduced by $86 \%$ in the $1: 2$ blend compared to pristine (1:0) polymer. Excitations close to resonance facilitate inter-molecular charge transfer between the polymer and PCBM. Farther above resonance the times converge to a value of 170 as for all blends including pristine polymer, as in this regime inter-polymer charge transfer dominates. In $\mathrm{MoS}_{2}$ charge transfer dynamics have been shown to vary depending on the morphology of the sample, including a single crystal, nanoparticles, and a composite of $\mathrm{MoS}_{2}$ sandwiching a graphene backbone, using CHCS [170]. While they are similar for the crystal and nanoparticle cases, owing to the local character of the probe, the composite exhibits a bi-modal charge transfer time distribution as a function of excitation energy. The switch between to regimes of charge transfer times is attributed to the core excited electron having sufficient energy to overcome the Schottky barrier created in the $\mathrm{MoS}_{2}$ /graphene interface.

CHCS has also been combined with polarisation-dependent HAXPES, which will be discussed in detail in section 3.2.2. Using linearly polarised hard $\mathrm{x}$-rays the charge transfer anisotropy upon resonant core-excitation has been recorded to study a layered $\mathrm{SnS}_{2}$ single crystal [171]. This delivers not only chemical specificity, but also orbital specificity through the difference in alignment of the $p_{x}, p_{y}$ and $p_{z}$ orbitals relative to the photoemission geometry. The resonant Auger spectroscopy maps recorded in normal and grazing emission can be directly compared to calculations of the band structure with orbital projections. CHCS can thus be used as a probe of the unoccupied band structure of a system using. Another example of combining linearly polarised light with CHCS is the case of black phosphorous explored by Johansson et al [172]. Black phosphorous is known to have different macroscopic conductivities in the armchair and zig-zag directions. This kind of interlayer charge transfer anisotropy can be studied with CHCS by aligning the crystal directions with the linearly polarised x-rays. Comparing charge transfer times in the two directions reveals that the armchair direction has a faster delocalisation of the core excited electron for excitation energies close to the $\mathrm{P} \mathrm{K}$-edge resonance. At about $2 \mathrm{eV}$ excess energy, the electron transfer time is equal for the two directions, but switches over to the zig-zag direction. Together with out-of-plane results a detailed picture of the electron dynamics using the orbital projected band structure can be achieved [168, 172].

Beyond the use of energy-dependent HAXPES for core level and Auger spectra it is a powerful tool to untangle the 

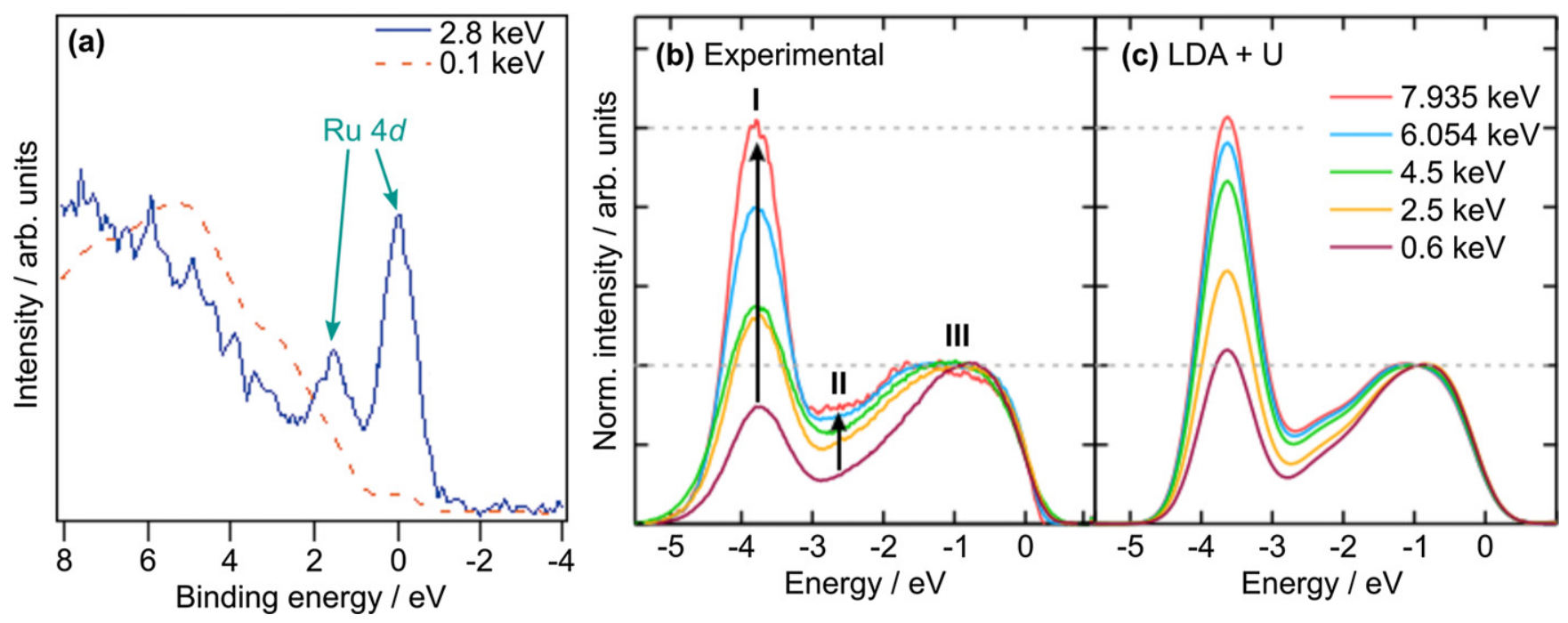

Figure 6. Energy-dependent HAXPES experiments of valence states. (a) Valence states of the ruthenium complex Ru(cbpy) ${ }_{2}(\mathrm{NCS})_{2}$ collected at 2.8 and $0.1 \mathrm{keV}$. The two Ru $4 d$ states from varying ligand coordinations can be observed using hard $\mathrm{x}$-rays. (b) and (c) Experimental valence spectra and LDA $+U$ theory results for CdO. Spectra are shown across a number of excitation energies, including a soft x-ray measurement at $0.6 \mathrm{keV}$ and four hard x-ray energies $(2.5,4.5,6.054$, and $7.935 \mathrm{keV})$. Reproduced from [176]. CC BY 3.0.

orbital composition of the valence states of molecules and materials [173]. The element-dependent changes in photoionisation cross section with excitation energy, allow the discrimination or enhancement of certain contributions to the valence electronic structure aiding interpretation and comparison with projected densities of states from DFT calculations. Examples of exploited variations in cross sections are relative decreases for the valence orbitals of light elements such as carbon, nitrogen, oxygen, and sulfur at higher energies, or the presence of Cooper minima for some transition metal valence orbitals. Thus comparing valence photoelectron spectra measured using varying excitation energies allows the characterisation of the frontier electronic structure.

An example for an experiment conducted on a molecular sample is shown in figure 6, where the valence states of the ruthenium complex $\mathrm{Ru}(\mathrm{cbpy})_{2}(\mathrm{NCS})_{2}$ is measured at two different photon energies [174]. The valence energy levels of this dye molecule are responsible for the light absorption properties and for the electron transfer reactions occurring during and after light absorption in photoelectrochemical processes. Only at higher photon energies can the $\mathrm{Ru} 4 d$ peaks, so important for the electronic structure of the molecule, be observed. Ru $4 d$ states show two peaks, as the complex is asymmetric with two different ligand coordinating the $\mathrm{Ru}$ atoms giving an orbital mix of $1: 2$. In contrast, in a symmetrical complex such as $\mathrm{Ru}(\mathrm{bpy}) 3^{2+}$ only one $\mathrm{Ru} 4 d$ feature is observed. Complementary studies determining contributions to the electronic structures from different ligands were further investigated using resonant photoelectron spectroscopy (RPES) [175]. This confirmed that the HOMO level is mainly of $\mathrm{Ru} 4 d$ for the $\mathrm{Ru}(\mathrm{bpy})_{3}{ }^{2+}$ complex. At the photon energy $2.841 \mathrm{keV}$ a clear resonance corresponding to excitation from $\mathrm{Ru} 2 p$ to unoccupied energy levels, resulting in enhanced emission of electrons from the $\mathrm{Ru} 4 d$ contribution in the valence electronic structure.
The detailed knowledge of the electronic structure of materials used in electronic and optoelectronic applications is crucial for the design of efficient heterojunctions in such devices. A clear example of the use of energy-dependent HAXPES in conjunction with DFT to gain an in-depth understanding of the electronic structure of a material is the study on n-type $\mathrm{CdO}$, a transparent conducting oxide (TCO) with great potential in photovoltaic applications, by Mudd et al [176]. Experimental results are used to verify different theoretical approaches for the applicability in the case of $\mathrm{CdO}$. Theoretical calculations of $\mathrm{CdO}$, like for many metal oxides, suffer from underestimation of the binding energy of shallow core states in close proximity to the valence band, often distorting the level of hybridisation present in such systems. Exact binding energy values for shallow core states and features in the valence band can be extracted from HAXPES and energy-dependent experiments enable the identification of the orbital character of specific features in the valence region. Figures 6(b) and (c) show the energy-dependent experimental spectra and photoionisation cross section corrected densities of states, respectively. Feature I at $-3.9 \mathrm{eV}$ shows a strong photon energy dependence, while feature III remains stable. Feature II also shows a response to changes in photon energy, which appears as a broadening of feature II. From these observed relative changes in the intensities of valence band features, and in conjunction with projected, photoionisation cross section corrected densities of states, Mudd et al are able to identify and discriminate between the varying contributions to the valence states. Using energydependent HAXPES it is also possible to observe and identify low energy plasmon features that fall within the valence states [177]. Another important aspect is that in valence band studies tuning of the photon energy allows precise definition of the momentum component $k_{z}$ perpendicular to the sample surface.

An important aspect to take into account when performing energy-dependent HAXPES is the occurrence of energydependent, recoil-induced binding energy shifts that may 
occur in both core and valence spectra. In the simplest form, the recoil energy $\Delta E$ can be estimated by the equation

$$
\Delta E=E_{\mathrm{K}}(m / M),
$$

where $E_{\mathrm{K}}$ is the kinetic energy of the ejected photoelectron, $m$ is the mass of the photoelectron, and $M$ is the mass of the atom the photoelectron originated from. In HAXPES experiments, $\Delta E$ can be observed as a shift of core or valence spectra toward higher binding. While recoil is observed routinely in gas-phase HAXPES of atoms and molecules (see section 3.3.1), observations in solids are still rare, as until recently it was assumed that because the momentum of photoelectron leaving an atom is transferred to the whole body of a solid the energy losses should be negligible. However, a small number of studies have shown that recoil-induced shifts can be observed in core level and valence band spectra, as well as at the Fermi edge in metallic samples [161, 178-181].

3.1.2. Angle-resolved HAXPES (HARPES). A wonderful advantage of HAXPES is, as mentioned before, the ability to probe deeper into the solid, thus more accurately measuring true bulk properties of materials and being able to access electronic-structural information from buried layers and interfaces. Such capability emerges due to the fact that photoelectrons with higher kinetic energies will travel farther within a solid and, therefore, more of the signal originating from the bulk will be detected by the analyser. This phenomenon is quantitatively described by the semi-empirical TPP-2M formula, which yields estimated values of effective attenuation lengths for most solids with reasonable accuracy [151, 182]. Such calculations predict roughly a 3-5 fold increase in the bulk-sensitivity of the measurement for most solids when going from $1 \mathrm{keV}$ to $10 \mathrm{keV}$ in excitation energy, facilitating measurements of multiple unit cells into the bulk, and therefore diminishing the necessity for careful surface preparation or in situ cleaving. The unique advantages of bulk-sensitivity and the possibility of using samples 'as prepared' is especially critical for crystalline and thin-film samples, where cleaving is difficult or not possible and the traditional surface preparation procedures do not preserve the original structure and composition of the surface layers. Thus, for momentum-resolved ARPES spectroscopy, which is typically carried out in the more surface-sensitive VUV regime (20-150 eV), going to higher photon energies not only provides a more accurate picture of bulk electronic structure, but also greatly expands the range of 'measurable' sample types.

The challenges of carrying out momentum-resolved ARPES-type valence band measurements at high photoexcitation energies have been investigated and discussed in-depth by Shevchik [183], Hussain et al [184], and later expanded upon by Fadley et al in several detailed overviews [41, 46]. The major fundamental challenge is associated with the increasing effects of the phonon-assisted non-direct transitions, for which thermal atomic vibrations diminish the degree of translational symmetry in the crystal and, therefore, suppress the dispersive direct-transition features that make up the band structure. The relative signal intensity of the 'useful' contribution originating from the direct electronic transitions could, with a good degree of precision, be estimated by a photoemission Debye-Waller factor, which is given by $W(T)=\mathrm{e}^{-\frac{1}{3} \vec{g}^{2}\left\langle U^{2}(T)\right\rangle}$, where $\vec{g}$ is the reciprocal lattice vector representing the change in the initial photoelectron wave vector due to a given direct transition, and $\left\langle U^{2}(T)\right\rangle$ is the one-dimensional mean-squared vibrational displacement at temperature $T$. Thus, as the $\vec{g}^{2}$ term grows due to the increasing excitation energy, the relative fraction of the dispersive features at the detector will diminish exponentially. This detrimental effect, however, could be countered by minimising the temperature-dependent $\left\langle U^{2}(T)\right\rangle$ term via cryogenic cooling of the sample, ideally, to liquid helium temperatures. Several recent experimental $[185,186]$ and theoretical $[187,188]$ studies explore the phonon-related effects in angle-resolved photoemission at high excitation energies and provide some material-specific guidelines for the practical implementation of such measurements.

Another fundamental challenge impeding a straightforward and widespread implementation of momentum-resolved valence band mapping in the multi-keV regime is the rapid decay of the photoionisation cross sections at high photon energies $[76,77]$. In a first-order approximation, the photoionisation cross sections diminish continuously with increasing photon energy, approximately as $E_{\text {kin }}^{-7 / 2}$ for $s$ subshells and as $E_{\text {kin }}^{-9 / 2}$ for $p, d$, and $f$ subshells. For the valence band states, such rapid decay of already relatively small cross sections results in orders-of-magnitude decreases of measured photoemission intensities. Due to this limitation, practical implementation of valence band HARPES had not been realised until the advent of third-generation synchrotron light sources and the development of third-generation undulators, high-resolution hard $\mathrm{x}$-ray monochromators, and focusing optics capable of producing highly intense ( $10^{11}$ to $10^{12}$ photons/s) beams. Equally important for this effort was the development of high-throughput analysers with carefully-matched acceptance areas and retarding optics tuned specifically for highly-energetic photoelectrons. Additional effects that become significant at higher photon energies and, therefore, must be accounted for include the shifts in momentum space due to the non-negligible photon momentum wave vector $\vec{k}_{h \nu}=2 \pi \nu / c$ [189], momentum-dependent intensity modulations due to the photoelectron diffraction [190], and energy-dependent shifts and smearing due to the recoil effects [178].

The first practical implementation of HARPES in the multi-keV energy regime was realised by Gray et al at the BL15XU beamline of the SPring- 8 synchrotron [191]. In order to minimise the phonon-related smearing effects, a singlecrystal of W with the (110) orientation was selected for the proof-of-principle experiments, due to its high atomic weight and high Debye-Waller factor at the routinely achievable liquid-helium temperatures of 20-30 K. For this temperature range, Plucinski et al had estimated the fraction of the momentum-conserving direct transition for W(110) to be approximately 0.45 for the excitation energy of $6 \mathrm{keV}$ [185]. At this energy, the average probing depth of approximately $6 \mathrm{~nm}$ for the valence electrons was predicted by the TPP-2M formula [182]. 

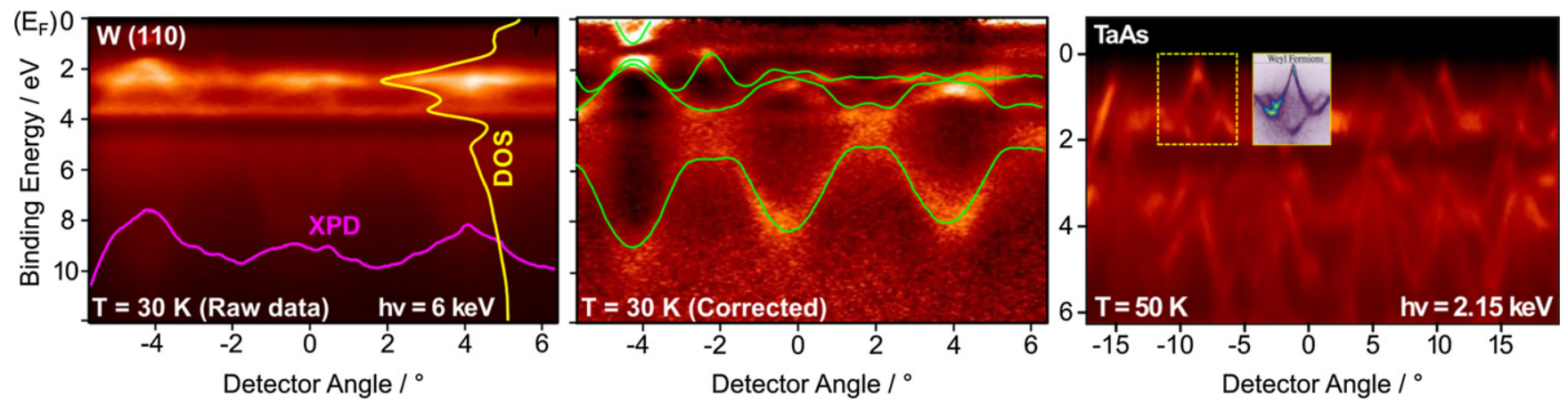

Figure 7. (a) Unprocessed HARPES spectrum for W(110) single crystal at a photon energy of $6 \mathrm{keV}$. The spectrum exhibits strong modulations due to the DOS in energy (yellow curve) and XPD effects in angle (magenta curve). Dispersive features are also observed owing to the considerable fraction (0.45) of direct transitions at the cryogenic temperature of $30 \mathrm{~K}$. (b) Data from (a), normalised by means of a two-step process so as to suppress the DOS and XPD effects, thus enhancing dispersive band structure features. The solid green curves superimposed on the experimental data are the results of free-electron final-state model band structure calculations. (a), (b) Reprinted by permission from Springer Nature Customer Service Centre GmbH: Nature [191] (2011). (c) Proof-of-principle HARPES measurements of the Weyl semimetal TaAs bulk band structure with $2.15 \mathrm{keV}$ excitation. The linearly-dispersive W1-type Weyl cone, clearly visible in the raw spectrum, is highlighted and compared to the recently-published data in the inset. (c) From [192]. Reprinted with permission from AAAS.

Figure 7(a) shows an unprocessed HARPES spectrum of $\mathrm{W}(110)$ recorded at the photon energy of $6 \mathrm{keV}$ and temperature of $30 \mathrm{~K}$. Although the dispersive bands are clearly visible across the entire detector image spanning approximately $12^{\circ}$, the spectrum also shows strong non-dispersive modulations due to the matrix-element weighted density of states (MEWDOS), which are represented by the yellow angle-integrated curve on the side. Additionally, strong angle-dependent modulations due to the x-ray photoelectron diffraction (XPD) effects are evident in the data and are represented by the energyintegrated magenta profile at the bottom of the image. By applying a two-step normalisation procedure to the raw spectrum using these angle and energy-averaged contours, one can correct in some way for both MEW-DOS and XPD-like effects in the data, and indeed the dispersing bands are much more clearly seen in the corrected figure 7(b). An obvious advantage of going to high excitation energies, beyond the deeper probing depth, is immediately evident-due to the large size of the final photoelectron wavevector $\vec{k}_{\mathrm{f}}$, a single detector image spans an extensive cross-section of the momentum space, providing band structure information for over three Brillouin zones in one shot. Furthermore, the agreement of the experimental dispersion with the band structure calculated using firstprinciples free-electron final-state theory (green contours) is quite reasonable, apart from minor excursions that were identified to be caused by a small $\left(<1^{\circ}\right)$ misalignment from the ideal experimental geometry.

Following these proof-of-principle demonstrations, HARPES has been utilised in conjunction with angleintegrated HAXPES to investigate various technologicallyrelevant materials, such as the dilute magnetic semiconductor $\mathrm{Ga}_{1-x} \mathrm{Mn}_{x}$ As $[193,194]$ and the thermionic material $\mathrm{LaB}_{6}$ [195], which is used extensively for hot cathodes in electron microscopy and lithography. Furthermore, a potential future application of HARPES to the studies of bulk valence band structure of quantum materials has been recently demonstrated by Gray et al with the measurements of the three-dimensional Weyl semimetal TaAs (see figure 7(c)) at the I09 beamline of the Diamond Light Source. The highlighted region near the Fermi level is compared to the recently-published data [192] revealing the linearly dispersive W1 Weyl cone (see inset) and demonstrates potential applicability of HARPES to the future studies of relevant spectroscopic features in novel topological materials. Thus, the future undoubtedly holds many more exciting developments in the field of HARPES. To this end, recent advances in instrumentation, such as photoelectron momentum microscopy discussed in the following section, and imminent future implementations of this technique at free-electron lasers will lead to the addition of new dimensions, such as time and spin. Equally important for these efforts will be new advances in theoretical treatment and interpretation of the angle-resolved data, including considerations of temperature, disorder, and electronic correlations, as well as accurate modeling of the XPD effects.

3.1.3. Hard x-ray momentum microscopy. Hard x-ray photoelectron momentum microscopy provides a novel approach to extend the capability of PES measurements in the hard x-ray regime, with a key focus on improving techniques such as HARPES and hXPD. This approach involves replacing the conventional hemispherical dispersive analyser, a hallmark of all conventional PES techniques for a ToF analyser in combination with a cathode lens-based system, otherwise known as a ToF momentum or ToF $k$-microscope [141]. A comparison of the two systems is schematically shown in figure 8 . The ToF system, as displayed in figure 8(a), has been designed to overcome the limitations that hard $\mathrm{x}$-rays bring, in particular the low photoionisation cross sections and reduced photon flux. The lens system consists of a series of zoom optic sections with a controllable aperture, and fronted with an objective lens to transform the incoming electron cloud into the required drift energy with maximum $k$-resolution. These lenses allow for the full-field imaging of Brillouin zones. The ToF column sits completely separate to the lens system consisting of a low energy drift section and a time-resolving imaging detector. This allows for the decoupling of the energy and 
(a)

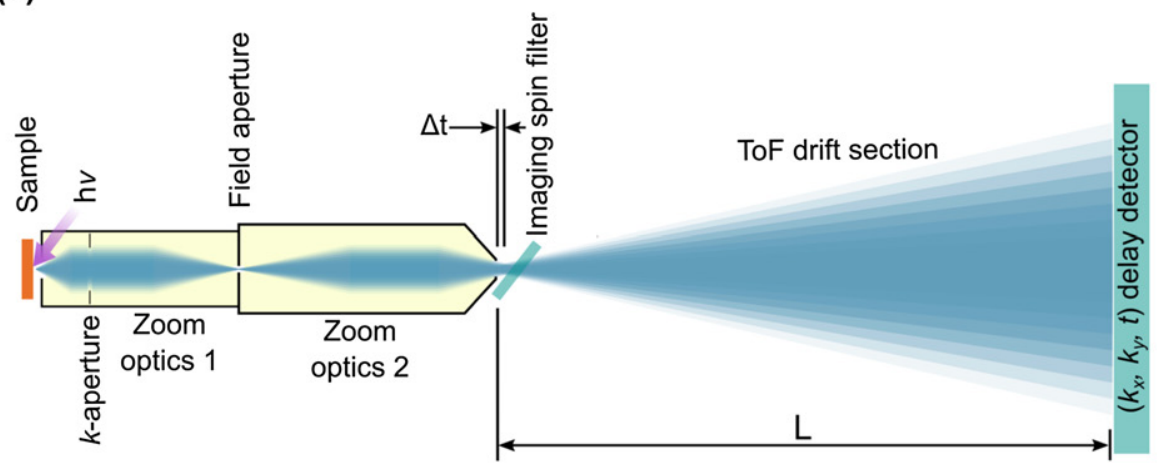

(b)

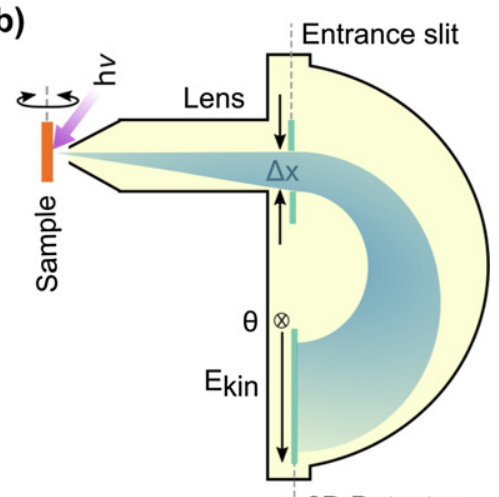

Figure 8. Schematics of HAXPES recording schemes, comparing the (a) $k$-imaging ToF spectrometer, with the (b) conventional hemispherical dispersive analyser. In both cases, electrons are generated in response to an incoming photon source of energy, $h \nu$ and captured through a series of lenses before entering the analyser and reaching the detector. The delay-line detector of the ToF spectrometer, displayed in (a) enables $3 \mathrm{D}$ data arrays $I\left(E_{\mathrm{kin}}, k_{x}, k_{y}\right)$ to be collected in the absence of beam-conflicting slits present in the hemispherical analyser shown in (b). The hemispherical analyser system shown in (b) cannot record $3 \mathrm{D}$ arrays, but rather $2 \mathrm{D}$ arrays $I\left(E_{\mathrm{kin}}, \theta\right)$. The angular term, $\theta$ is adjusted either through sample rotation or adjusting the deflectors within the electrostatic lens system.

momentum coordinates; this coupling hampered the use of the dispersive analyser. The microscope system is able to provide full field imaging of the photoelectron distribution in the momentum domain, while the ToF analyser offers high parallelisation, decreasing the acquisition time and increasing the dimensionality of the recording scheme. The energy resolution of the hemispherical analyser is governed by the entrance slit width $(\Delta x)$, as shown in figure 8(b) and pass energy. A sufficiently narrow entrance slit is required to achieve the desired energy resolution and the slit design provides a series of challenges to obtain this. The ToF analyser overcomes the energy resolution limits of the dispersive analyser by omitting the slit design, in favor of a 'thin' isochrone surface with a much narrower entrance width $(\Delta t)$. Such a design is only feasible as the ToF spectrometer demands pulsed excitations, which is not the case for the hemispherical analyser. In the ToF system, the energy resolution is determined by the length of the ToF drift section $(L)$, drift energy and the time resolving capability of the detector. A critical component of the detection system is the three-dimensional $\left(k_{x}, k_{y}, t\right)$-resolving delay-line detector (DLD), which facilitates the high speed recording necessary to maximise parallelisation and captures several hundred energy slices across a given binding energy range. Additionally, it is the $3 \mathrm{D}$ recording scheme achieved with the DLD that efficiently helps to overcome the limits provided by hard $\mathrm{x}$-rays in HAXPES measurements, as previously mentioned [196].

The ToF microscope offers the opportunity to directly image in momentum space, which can provide the opportunity to acquire valence band maps in full $k$-resolution, enabling the study of important features such as band dispersion or the shape and topology of the Fermi surface of a material. Accessing the hard $\mathrm{x}$-ray regime also brings an added benefit. In this regime, the wavelength of the outgoing electrons can approach and shorten to less than the inter-atomic distances, giving rise to diffraction signatures, which can strongly modulate the resultant band structure pattern but also provide valuable structural information. Therefore, the application of the ToF momentum microscope operating with high photon energies can provide both electronic structure information as well as geometric structural information, with the latter in the form of x-ray photoelectron diffraction (XPD) patterns.

The ToF set-up has shown great promise in the VUV and soft x-ray regimes [197-199]. Medjanik et al demonstrated the first instance of applying this novel ToF $k$-microscope to fully map the bulk electronic structure of tungsten in the soft $\mathrm{X}$-ray regime [141]. In comparison to conventional ARPES with a dispersive analyser, this approach greatly simplified the process route to map the 4D spectral density function. Additionally, the ToF analyser offers approximately two orders of magnitude faster acquisition time in comparison to the hemispherical analyser, with the recorded counts in the millions per second range.

Soon after these benchmark soft x-ray ToF $k$-microscopebased ARPES experiments, there appeared to be an immediate desire to carry out measurements with this instrument set-up in the hard $x$-ray regime. The initial HAXPES experiments were conducted at beamline P22 of PETRA III, using a new lens system, which offered a larger $k$-field of view and could detect electrons with a kinetic energy up to $8 \mathrm{keV}$ [57]. This enabled access to the collection of X-ray photoelectron diffraction (XPD) patterns, further increasing the level of information available from the technique. Publications since the pilot studies have continued to exploit the microscope to study the valence band structure or to acquire XPD information of a range of materials and some are discussed in detail below [137, 197, 200-205].

Initial studies in the HAXPES regime were conducted by Medjanik et al and presented proof of principle of the instrument by successfully mapping the electronic structure of $\operatorname{Re}(0001)$ at $h \nu=3.83 \mathrm{keV}$ in a similar manner to the soft $\mathrm{X}$-ray measurements conducted on W(110) prior [196]. Spacecharge effects stemming from the Coulomb interaction of the photoelectron cloud induced an energy shift and an anisotropic 
acceleration of the electrons, which led to a Lorentzian deformation of the spectra. However, this deformation was reported to be significantly less observable compared to the soft x-ray measurements and could be removed by applying correction algorithms [202]. Photoelectron diffraction signatures were also shown to imprint on the valence band maps, modulating the results in the form of high intensity spikes or dark lines. Babenkov et al also observed similar modulations when studying both valence band structure and core levels of $\mathrm{Mo}(110)$ and layered $\mathrm{TiTe}_{2}$ using HARPES with the momentum microscope set-up, whereas Schönhense et al observed them in valence band photoelectron diffractograms across a range of transition metals [200, 201]. The main modulations are attributed to Laue diffraction, intrinsic to the photoemission process and the more dominant Kikuchi-type diffraction which are extrinsic to the photoemission process. The Kikuchi diffraction effects are considered to have a long range modulation of the valence band pattern and core level XPD, and contribute to the underlying quasi-elastic background. Kikuchi patterns are generated due to the stochastic nature of electron scattering events in which spherical waves form and are diffracted inside the material, therefore the Kikuchi patterns mirror the full lattice symmetry. Schönhense et al developed a method of reducing the Kikuchi modulations from valence band $k$-distributions using a multiplicative approach involving the pixel-by-pixel division of the raw valence band maps by the simultaneously collected XPD patterns. This was shown to substantially improve the valence band maps, with band structures more pronounced and distortion reduced [201, 202].

Fedchenko et al presented the first instance of using the momentum microscope directly for its diffraction capability in the hard $\mathrm{x}$-ray regime rather than its valence band mapping capability [203]. Core level XPD were conducted on single crystal graphite across a photon energy range of $2.5-7.3 \mathrm{keV}$ with a focus on the C $1 s$ core level. Results were compared with dynamical calculations in an attempt to correlate diffraction with the experimentally observed inelastic scattering events, namely the well-known generation of Kikuchi band features. The theoretical method employed a Bloch-wave approach to quantitatively simulate the diffraction of electrons from the atomic emitters. The Bloch-wave approach has the key benefit of better predicting long-range order which is often the shortcoming of the commonly used spherical-wave cluster approach. The benefit of the microscope is the data acquisition process that allows for the extraction of multiple diffractograms from a single three-dimensional data array. The patterns exhibited significant domination from Kikuchi bands projected from the Bragg reflection on the (110) lattice planes. Excellent agreement between theory and experiment was observed showing that the Bloch-wave approach was capable of describing the diffractograms and aided with the disentanglement of the fine structure in the patterns. Fedchenko et al extended the application of this combined experimental hXPD ToF $k$-microscope and theoretical Blochwave approach to conduct emitter-site specific structural analysis in Te-implanted $\mathrm{Si}(001)$ [204]. Excellent agreement between the experimental Te $3 d$ hXPD patterns and the calculated Kikuchi patterns were reported. By analysing the Kikuchi band structure position and intensity within the core level diffractograms, it was observed that the effective dopant concentration varied between the probed bulk volume and the nominal concentration, attributed to the anisotropic signature of the ion implantation process [205].

More recently, Kutnyakhov et al applied the ToF $k$ microscope set-up to extend momentum-resolved PES into the time domain, giving rise to what is known as time-resolved momentum microscopy (trMM) [137]. These measurements were conducted at the PG2 beamline at FLASH (DESY, Hamburg) within the soft X-ray regime (24-885 eV) on bulk and layered $\mathrm{WSe}_{2}$, presenting both core level and valence photoemission spectra. This marks one of the first instances of using the novel ToF $k$-microscope instrument for XFEL time resolved measurements and was made possible through work provided by the Mainz group, who developed suitable theoretical models and correction algorithms to overcome the limits of the pump-probe scheme [202]. Coupling the multidimensional energy recording scheme of the TOF $k$-microscope with the pump-probe scheme of XFELs, extends the technique into the time domain, with femtosecond resolution. However, as is the case with trHAXPES, space-charge effects still arise due to the pump-probe scheme. Future developments may look to extend trMM into the hard x-ray regime to study the ultra-fast dynamics within the bulk of a material [129].

Being deep bulk sensitive, hard x-ray ARPES and momentum microscopy for ferromagnetic and antiferromagnetic materials are highly attractive, because the results are not influenced by surface effects. An emerging field along this line is the response of the electronic structure on mechanical strain or on the orientation of the magnetisation vector or the alignment of the Néel vector. As an example, we show a recent measurement that is of current interest both from the fundamental point of view and for future applications in spintronics devices. A central question for antiferromagnets is whether a reorientation of the antiferromagnetic order parameter in real-space causes significant changes of the band structure in $k$-space. This research is fueled by predictions of metal-insulator transitions and Dirac quasiparticles in specific antiferromagnets [206]. Antiferromagnets are favorable for such concepts because of their symmetric spin-dependent density of states.

Sizable changes of the electronic band dispersion close to the Fermi energy upon Néel vector realignment were recently observed for epitaxial $\mathrm{Mn}_{2} \mathrm{Au}(001)$ films, see figure 9 [207]. Such experiments are hampered by the lack of a method for in situ switching of the Néel vector. The situation is different for ferromagnets, which can easily be switched by magnetic field pulses. A reliable way to realign the Néel vector in antiferromagnets is to exploit the spin-flop transition, induced by strong field pulses (60 $\mathrm{T}$ in the measurements of figure 9). Field pulses of this amplitude cannot be applied in situ and only special laboratories provide such high fields. This situation seemed prohibitive to study the interplay of band structure and Néel-vector alignment. Hard x-ray photoelectron spectroscopy offers a loophole out of this dilemma: the samples are covered by a protective coating, which allows transport and field-treatment under ambient air and subsequent hard x-ray 


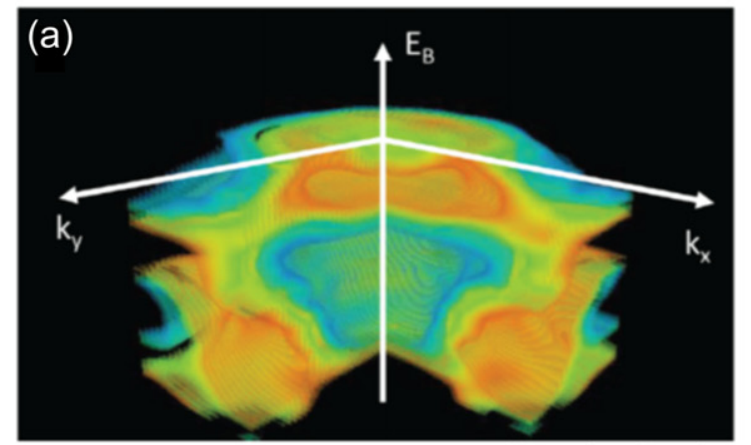

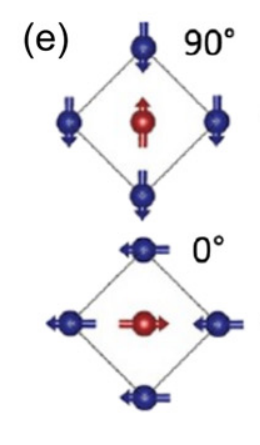

(f)
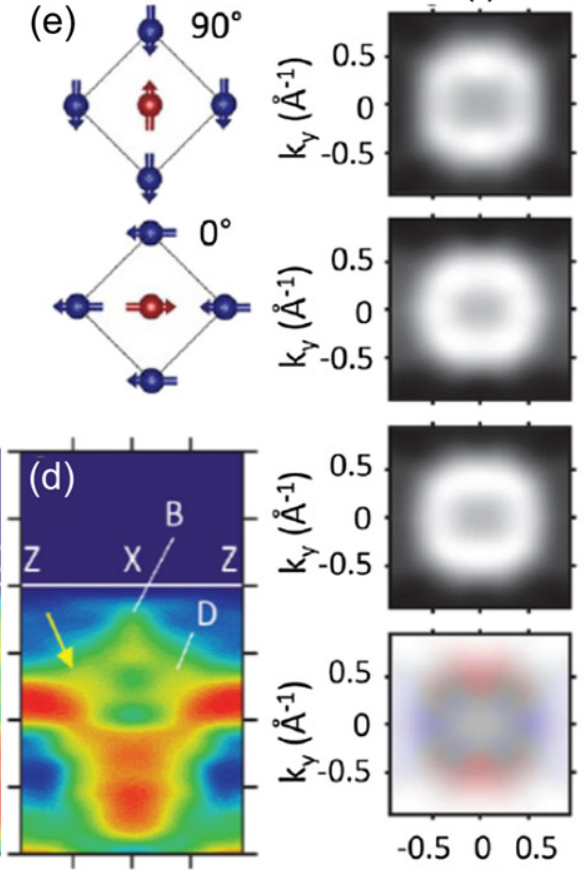
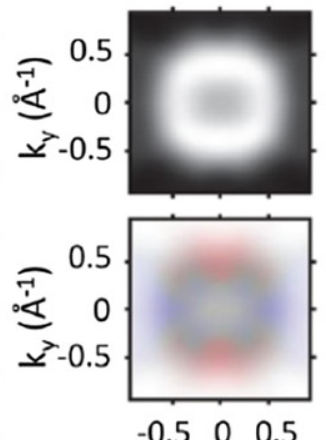

$k_{x}\left(\AA^{-1}\right)$ (g)
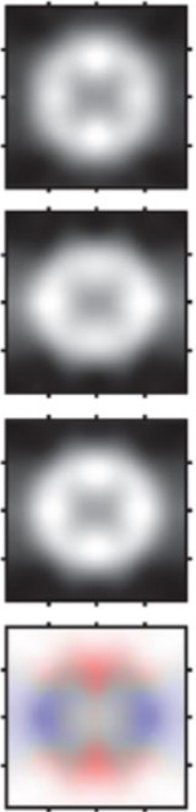

$\begin{array}{lll}-0.5 & 0 & 0.5\end{array}$

$k_{x}\left(\AA^{-1}\right)$ (h)
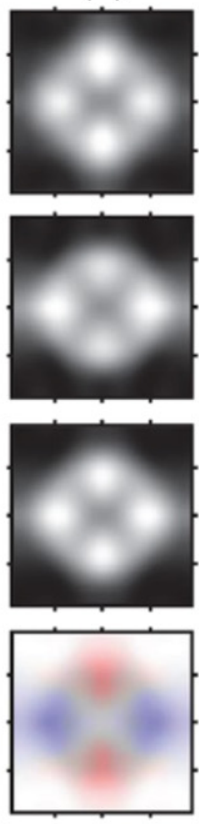

$\begin{array}{lll}-0.5 & 0 & 0.5\end{array}$

$k_{x}\left(\AA^{-1}\right)$

Figure 9. Hard $\mathrm{x}$-ray momentum microscopy measurement at $h \nu=5.23 \mathrm{keV}$ for the antiferromagnet $\mathrm{Mn}_{2} \mathrm{Au}$; $40 \mathrm{~nm}$ epitaxial film with $2 \mathrm{~nm}$ protective Ta capping. The Néel vector was aligned by a $60 \mathrm{~T}$ field pulse at $77 \mathrm{~K}$, applied ex situ. (a) View of the measured data array $I\left(E_{\mathrm{B}}, k_{x}, k_{y}\right)$ with cutout to see inner part. (b)-(d) $k_{x}-k_{y}$ section at $E_{\mathrm{F}}, E_{\mathrm{B}}-k_{x}$ section (along $\left.X \Gamma X\right)$ and $E_{\mathrm{B}}-k_{y}$ section (along ZXZ), respectively. (e) Perpendicular alignment of the Néel vector along the directions $0^{\circ}$ and $90^{\circ}$. (f) - (h) $k_{x}-k_{y}$ sections for $90^{\circ}$ alignment (top row), for $0^{\circ}$ alignment (2nd row), average of $0^{\circ}$ and $90^{\circ}$ (3rd row) and asymmetry (bottom row); binding energies $E_{\mathrm{B}}=0$ (f), $0.15 \mathrm{eV}$ (g) and $0.25 \mathrm{eV}$ (h). Reprinted with permission from [207]. Copyright (2020) American Chemical Society.

momentum microscopy looking through the capping layer (beamline P22 of PETRA III).

Figure 9 shows (a) a view of the $3 \mathrm{D}$ data array $I\left(E_{\mathrm{B}}, k_{x}, k_{y}\right)$, (b) a $k_{x}-k_{y}$ section at $E_{\mathrm{F}}$, and (c) and (d) $E_{\mathrm{B}}-k_{x, y}$ sections along $X \Gamma X$ and $Z X Z$, respectively, showing the band dispersions. The photon energy of $5.23 \mathrm{keV}$ probes the $\Gamma X \Sigma$-plane of the Brillouin zone. The Néel vector is oriented along the $k_{x}$ or $k_{y}$ direction (denoted as $0^{\circ}$ and $90^{\circ}$ in (e)), both corresponding to the $\langle 110\rangle$ easy direction. Close inspection of the $k_{x}-k_{y}$ patterns at three different binding energies (f) -(h) reveals clear differences, quantified by the asymmetry $\left(I_{90}-I_{0}\right) /\left(I_{90}+I_{0}\right)$. Red and blue denote higher intensity for $90^{\circ}$ and $0^{\circ}$ alignment, respectively. The asymmetry is largest at $E_{\mathrm{B}} \simeq 0.15 \mathrm{eV}$, reaching $10 \%$. This asymmetry can be explained by the broken inversion symmetry of the $\mathrm{Mn}_{2} \mathrm{Au}$ lattice (paramagnetic space group $I 4 / \mathrm{mmm}$ ). The antiferromagnetic order reduces the space group to $P 4 / \mathrm{m}^{\prime} \mathrm{mm}$, and further to $\mathrm{Fm}^{\prime} \mathrm{mm}$ after inclusion of spin-orbit interaction. The antiferromagnetism in $\mathrm{Mn}_{2} \mathrm{Au}$ represents a special type of inversionsymmetry breaking, which is different from structural chiral symmetry breaking: it introduces coupling of the energy bands to the Néel vector direction. $\mathrm{Mn}_{2} \mathrm{Au}$ exhibits a magnetic toroidal dipole Fermi surface, i.e., a spin-degenerate Fermi surface, which is not symmetric around the $\Gamma$-point [208]. These anisotropies can lead to large magnetoresistance, transport and topological effects. In this experiment the postmonochromator was not yet in operation, hence the photon bandwidth of $170 \mathrm{meV}$ was the limiting factor for energy and momentum resolution. In addition, the mosaicity of the film, revealed by x-ray diffraction, causes a Gaussian blurring of the bands. The results show good agreement with photoemission calculations (for details see reference [207]).

Overall, ToF momentum microscopy provides a number of benefits. Most notably it can offer tomographic $k$-space mapping of the valence electronic structure as well as providing both core level and valence band spectra that carry geometrical information, in the form of XPD spectra. The main challenges for the future involve developing a better understanding of space charge effects and how to best reduce them, but also the development of suitable data harvesting methods is required to accommodate the large volumes of data obtained by the measurement. Given the latest drive in developing strategies for correcting and minimising modulations, the technique holds great promise for the future of HARPES, hXPD and their time-resolved variants.

3.1.4. Hard $x$-ray photoelectron diffraction ( $h X P D)$. In $x$-ray photoelectron diffraction (XPD) the outgoing photoelectron wave with origin at the selected emitter-atom species is diffracted at the lattice. Early work on XPD appeared at about the same time as first fundamental work [209-211]. For several decades, XPD measurements have been extensively employed for structural analysis, mostly using hemispherical analysers in combination with special sample goniometers for 
precise rotation. A series of excellent reviews on this topic has been published to date [212-218]. Much younger is the technology for recording full-field imaging XPD, either employing display-type devices with combinations of grids [219-224] or using momentum microscopy with ToF energy discrimination [196]. Here, the new developments toward imaging XPD in a momentum microscope, recently extended to the hard X-ray range (hXPD), are summarised. The large IMFP in the hard $\mathrm{X}$-ray regime yields a large probe volume, comprising $10^{5}$ to $10^{6}$ atoms [182]. In this regime the long-range order is probed and the wavelength of the outgoing photoelectrons is very short, which emphasises high structural sensitivity. The increasing sharpness and sensitivity with increasing energy is clearly visible in the sequence of hXPD patterns for graphite between $E_{\text {final }}=2.6$ and $7 \mathrm{keV}$ [203].

XPD differs in several respects from conventional diffraction. The most important practical aspect is the element specificity based on addressing a specific core level. The characteristic binding energies of inner shells allow to select a certain atomic species, either inside the bulk or at the surface of a material. Chemical shifts due to different local configurations, e.g. of dopant atoms in a host lattice, can be exploited in order to disentangle non-equivalent coexisting sites. Emitter atoms on different lattice sites lead to markedly different diffraction patterns, which can be utilised for a fingerprint-like identification of dopant sites [204, 225].

A more fundamental aspect concerns the electron diffraction from the periodic potential of a lattice. According to Friedel's law, the Fourier transform of the real function describing the lattice obeys the symmetry property $F(k)=F^{*}(-k)$, where $F^{*}$ is the complex conjugate of $F$. In a conventional diffraction experiment, the intensity pattern is given by the squared amplitude $\left|F^{2}\right|$, which is identical for the Friedel pairs $(k,-k)$. In the XPD case only a selected atomic species in a dissymmetric lattice structure acts as 'internal source'. This circumvents the precondition for the validity of Friedel's law, hence a missing inversion center becomes visible in an XPD pattern. An example is shown in figures 10(a) and (b), visualizing the missing inversion center of GaAs by comparison of the Ga $3 d$ and As $3 d$ hXPD diffractograms. This prototypical semiconductor crystallises in the non-centrosymmetric zinc blende structure. For a detailed comparison, the photon energy is tuned such that the $\mathrm{Ga}$ and As core-level signals are recorded at identical kinetic energies and also the microscope settings are kept constant. Then it is possible to form ratio images, strongly emphasizing the differences (figure 10(c)). Note that Kikuchi bands provide a metric for $k$-space, see scale bar in figure 10(b). For further details, see [205].

There are two different theoretical models describing XPD. Firstly, the cluster-type approach $[226,227]$ treats the multiple-scattering scenario of sufficiently many atoms in real space. Such calculations do not rely on long-range periodic order and are thus particularly powerful for surface structures and adsorbates [228]. A well-established code is the EDAC program by Fadley and Garcia de Abajo [227]. Secondly, the Bloch-wave approach treats photoelectron diffraction (Bragg reflection) from lattice planes. Its elegance lies in the simplification that a large number of scatterers within the interaction volume (up to $10^{6}$ ) is represented by a small number $(<100)$ of relevant sets of lattice planes, parametrised by reciprocal lattice vectors. This Ansatz relies on long-range periodic order and is thus not applicable to surface structures, making both models nicely complementary. A comparison of both approaches is given by Winkelmann et al, who also proved that both methods yield identical results when fully converged [229].

Technically, Bloch-wave calculations exploit the fact that photoemission can be considered as a time-reversed LEED state' as introduced by Pendry for low energies [230-232]. In the calculation, an incoming plane wave is coupled to the Bloch wave field inside of the crystal. Thanks to the reciprocity theorem, hXPD patterns for arbitrary positions and numbers of emitter atoms in the unit cell are calculated simultaneously, making theoretical hXPD analysis a very fast method. Figures 10(f)-(j) show hXPD patterns calculated using the Bloch-wave approach. The agreement of figures 10(f)-(h) with experiment figures 10 (a)-(c) is excellent. Strikingly good agreement between measured hXPD diffractograms and Kikuchi patterns calculated by the Bloch-wave approach have also been found for graphite [203] and Si [204, 225], i.e. for light elements with their relatively small scattering factors. For a heavy material like Re the measured diffractograms are not dominated by Kikuchi lines, although Kikuchi bands can still be identified [196].

One of the promising applications of the young technique of full-field hXPD is the determination of dopant sites in semiconductors. It is expected that several competing sites coexist, open questions concern the hyper-doped regime, where multimer formation is expected. A material class with a high potential for applications are the dilute ferromagnetic semiconductors, a prototypical member being ( $\mathrm{Ga}, \mathrm{Mn}) \mathrm{As}$. The dissymmetric hXPD patterns of Ga and As (figures 10(a) and (b)) are perfect as reference fingerprints for substitutional dopant sites. The excellent agreement with Bloch-wave calculations opens the door to use calculated fully arbitrary dopant configurations, which are then compared with experiment. Various interstitial sites, dimers (with and without relaxation), trimers and clusters with different numbers of emitter atoms and different orientations with respect to the host lattice can be calculated without much additional computational effort. In general, such sites and configurations show very different hXPD signatures. Figures 10(i) and (j) show the hXPD fingerprints of tetrahedral and hexagonal substitutional sites in the GaAs lattice. The measured hXPD pattern of Mn shows that up to a concentration of $5.5 \%$ the $\mathrm{Mn}$ atoms occupy predominantly the Ga substitutional sites. For a detailed study of many different sites in the Si lattice, see [204], which allowed to identify different coexisting sites of Te atoms in $\mathrm{Si}$ in the hyper-doped regime [225].

For sake of conciseness we will only briefly mention further important aspects of photoelectron diffraction. High-quality photoelectron diffractograms can be considered as atomic holograms [233]; a refined theoretical treatment has been published very recently [234]. Since scattering cross sections are generally spin dependent, spin-polarised photoelectron 
$\mathrm{Ga} 3 d_{5 / 2}$

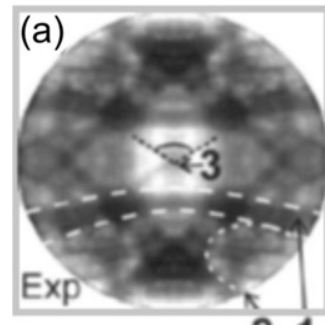

21

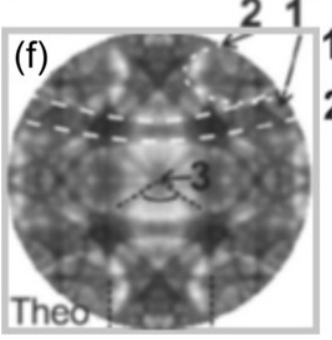

As $3 d_{5 / 2}$
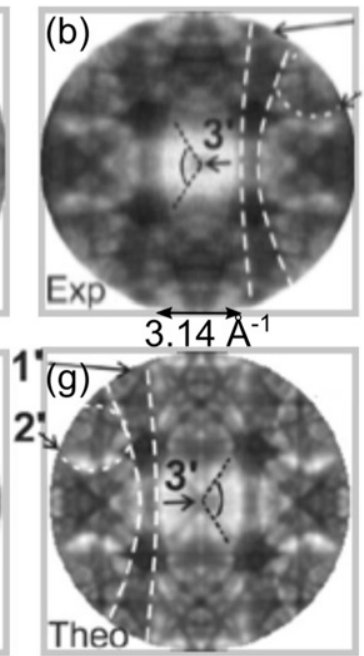

As $3 d / \mathrm{Ga} 3 d$ ratio images
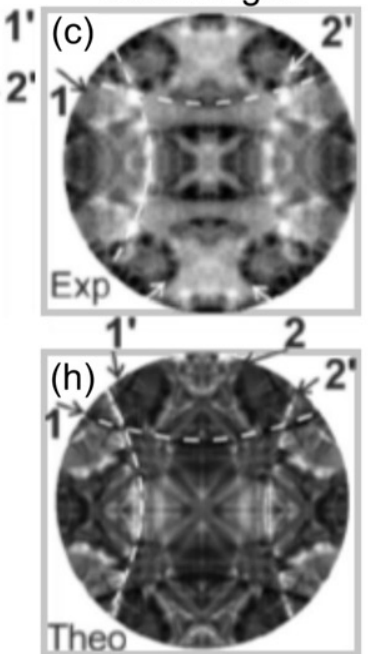

$\mathrm{Mn} 3 d_{5 / 2}$
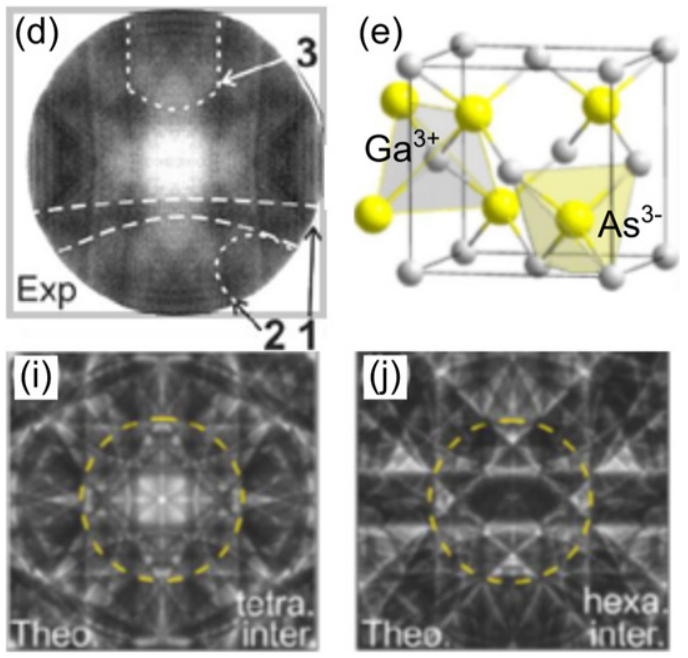

Figure 10. Measured diffraction patterns of photoelectrons from Ga $3 d_{5 / 2}$ (a) and As $3 d_{5 / 2}$ (b), corresponding ratio image (c) and pattern for Mn 3d $d_{5 / 2}$ (d). Data were recorded for a high-quality MBE grown GaAs(001) thin film doped with 3\% In and 2.5\% Mn. Identical

$E_{\text {kin }}=3.31 \mathrm{keV}$ was set by tuning the photon energy to $3317 \mathrm{eV}$ for Ga (a), $3340 \mathrm{eV}$ for As (b) and $3355 \mathrm{eV}$ for Mn (d). (e) GaAs unit cell with indicated tetrahedral coordination of the $\mathrm{Ga}^{3+}$ and $\mathrm{As}^{3-}$ ions (non centrosymmetric zinc-blende lattice). Note that all Ga and As tetrahedra are rotated by $90^{\circ}$ with respect to each other. (f)-(h) Calculated Kikuchi patterns corresponding to (a)-(c) and (i), (j) calculated fingerprint hXPD patterns for Mn atoms on tetrahedral (i) and hexagonal interstitial sites (j). All calculations for $E_{\text {kin }}=3.31$ keV (final-state energy inside the material). Dotted lines and numbers mark characteristic features; the dashed circles in (i), (j) denote the field-of-view of (a)-(c). Data previously published in reference [205].

diffraction can uncover the short-range magnetic order $[235,236]$. It was found that Kikuchi-type XPD signatures are imprinted on the band patterns in HARPES experiments and also on the underlying inelastic background (originating from thermal diffuse scattering). These signatures can largely be eliminated by multiplicative correction using a reference corelevel diffractogram, measured at the same kinetic energy [200]. In addition, Laue-type diffraction is imprinted on the valence band patterns, so that a full analysis of valence band XPD requires a momentum-transfer treatment including the photon momentum that is transferred to the valence photoelectrons [201]. An emerging application of XPD is its timedependent variant in femtosecond pump-probe experiments [129, 137, 237]. Quasi-simultaneous recording of valence band patterns, transient core-hole screening and coherent phonons in the same experiment with fs $\mathrm{x}$-ray excitation allows an unprecedented view into the interaction of transient lattice distortions and electronic states, being one of the central science cases in the field of quantum materials. Access to these types of experiments has been made possible due to the recent developments of HAXPES at XFELs (see section 2.3).

\subsubsection{Angular-dependent HAXPES (AR-HAXPES). The} advent of photoelectron analysers with large acceptance angles has enabled the development and application of angular dependent HAXPES (AR-HAXPES). In this type of experiment the angular distribution of the photoelectrons across different take-off or photoemission angles $\theta$ with respect to the surface normal is measured while keeping a fixed incident angle and sample position. As the effective electron escape depth $\lambda_{\text {eff }}$ depends on both the inelastic mean free path $\lambda$ and the take-off angle $\theta$, following $\lambda_{\text {eff }}=\lambda \cos \theta$, going to larger $\theta$ angles corresponds to a decrease in $\lambda_{\text {eff }}$ obtaining more surface sensitive data sets. An advantage of performing AR-HAXPES to obtain depth-dependent information is that in contrast to energy-dependent measurements, the photon energy is kept the same across all measurement points.

Beamline BL47XU at SPring-8, Japan, has been an early trailblazer in AR-HAXPES since their implementation of a Scienta R4000 analyser coupled with a wide acceptance angle electrostatic lens (WAAEL) [68]. This system has an effective acceptance angle of approximately $\pm 30^{\circ}$ and an angular resolution of $1.321^{\circ}$. Since 2013 a considerable number of studies performed at the beamline using an x-ray energy of $7.94 \mathrm{keV}$ have provided insights into predominantly structured, multilayer samples for device applications [68, 238-240]. AR-HAXPES was identified as a useful technique to study such multilayer systems from the beginning, including the first HAXPES paper at SPring- 8 which explored the $\theta$ dependence of the $\mathrm{Si} 1 s$ spectra of a $\mathrm{HfO}_{2} / \mathrm{SiO}_{2} / \mathrm{Si}$ system [29].

Of particular interest in such studies are changes to the band positions at interfaces, including band bending and rigid band edge shifts, which can be inferred from core level shifts as a function of distance from a surface or interface. Sato et al for example explored the interaction of nanoscale metal particles, including $\mathrm{Pt}, \mathrm{Au}$ and $\mathrm{Rh}$, with $\mathrm{TiO}_{2}$ [238]. In combination with DFT calculations they were able to identify electrostatic interactions taking place, which influenced not only the surface and interfaces, but the bulk properties of $\mathrm{TiO}_{2}$. This study showcases the crucial information AR-HAXPES can provide on device-relevant structures. Another example of studying 
the electronic structure around material interfaces is the work by Sing et al who used the angular-dependence of the Ti $2 p$ core level in $\mathrm{LaAlO}_{3} / \mathrm{SrTiO}_{3}$ heterostructures to study the two dimensional electron gas (2DEG) at the interface [241].

Beyond interfaces in electronic devices, Ozawa et al presented another application area where AR-HAXPES proves useful for the study of buried interfaces [242]. They explore adhesive interfaces between natural rubber and copper-zinc alloys, such as brass, which are essential for the performance of e.g. automotive tires. In their study, they used a range of core level spectra of both the rubber and alloy to understand the chemical mechanisms that underlie adhesion between these materials and can explain both reinforcement and degradation in this composite system. They are able to distinguish e.g. differences in sulfur species located in the bulk of the rubber and those formed specifically at the interface.

It should be noted, that most studies to date solely use core level spectra to explore systems using AR-HAXPES, predominantly due to the loss in signal intensity in ARHAXPES compared to integrated spectra. However, a recent study by Oshime et al was able to explore the valence band spectra of $\mathrm{BaTiO}_{3}$ enabling the interpretation of its ferroelectric electronic structure [240]. AR-HAXPES can also be combined with polarisation-dependent HAXPES, as in an example reported by ViolBarbosa et al, who studied a ferromagnetic layer of $\mathrm{Co}_{2} \mathrm{FeAl}$ grown on MnIr and buried beneath a MgO layer [243]. Polarisation-dependent HAXPES will be discussed in more detail in section 3.2.2.

The examples included in this review of AR-HAXPES exploit the wide acceptance angle of the ScientaOmicron EW4000 analyser to obtain angle-dependent spectra. A new HAXPES end-station at beamline I09 at Diamond Light Source, which is currently being commissioned, has been developed following a different strategy. It is equipped with an SPECS Phoibos 225 analyser without a wide angle lens, offering a small acceptance angle of about $7^{\circ}$ in an angular mode at high kinetic energies. However, the end-station vessel, together with the analyser, is designed to be able to rotate about a vertical axis in $10^{\circ}$ steps over a range of $55^{\circ}$. This allows the angle between the electron analyser and the polarisation vector of the $\mathrm{x}$-ray beam to vary between 0 and $55^{\circ}$. Using the polar stage of the station's vertical sample manipulator to compensate this rotation, it should then be possible to maintain a grazing $x$-ray incidence angle with respect to the sample surface at the different electron collection angles. It is estimated that the data acquisition time of an AR-HAXPES measurement with such a setup will be comparable to the use of an EW4000 analyser mounted parallel to the polarisation direction, where one needs to adopt a $30^{\circ}$ incidence angle to cover a similar range of electron emission angle with the wide angle lens.

Beyond the synchrotron-based experiments presented so far, the first example of an AR-HAXPES study using a laboratory-based HAXPES system (Scienta Omicron HAXPES Lab) was recently reported by Nishihara and colleagues [88]. They successfully used the technique to probe the depth dependence of SiN chemical states across the depth of a $\mathrm{Si}$ trench in an integrated circuit structure. AR-HAXPES has also been demonstrated on the same system at the University of Manchester, where buried layers were explored and measurements compared to synchrotron measurements varying the photon energy. As expected, an increase in photoemission angle decreases the sampling depth, analogous to reducing the photon energy [113].

\subsubsection{Hard x-ray photoemission electron microscopy (HAX-}

PEEM). Photoemission electron microscopy provides a magnified image of the lateral intensity distribution of photoelectrons emitted by a sample. The technique is popular using UV and soft x-ray excitation and commonly provides lateral resolution in the range of several $10 \mathrm{nms}$ [244-246]. PEEM at higher, hard $\mathrm{x}$-ray excitation energies, termed HAXPEEM, is still a niche technique. However, for the analysis of microstructured and layered samples, where a high lateral resolution and a high probing depth are desired, HAXPEEM has great potential. The discussion here is restricted to energy-filtered HAXPEEM of high kinetic energy electrons, in line with the other aspects of HAXPES presented herein, and a concise overview of the technique and some of the results produced so far are presented.

The working principle of HAXPEEM builds upon the low energy electron microscope for which the use of electrostatic immersion lenses was pioneered by Bauer [250, 251]. An electrostatic immersion lens operating at high extraction voltages of up to $24 \mathrm{kV}$ is used to collect the full half space of emitted electrons. The sample is usually held at high voltages resulting in a strong field gradient, which accelerates the electrons toward the immersion lens system where a first direct image of the emitted electrons is formed after the objective lens. Electrons are subsequently retarded to the column potential and further magnification is achieved as the electrons pass several projection lenses. At this point an energy dispersive element is used (usually a hemispherical analyser) which acts as an energy filter and allows energy dispersive recording of electron kinetic energies $\left(E_{\mathrm{K}}\right)$. The electron energy is scanned by variation of the sample potential and thereby quantitative XPS data is recorded for each point of the sample. Such spectromicroscopy is only possible due to the combination of parallel imaging and high energy resolution. In general the imaging principle is very similar to momentum microscopy, presented in detail in section 3.1.3. The main difference being that here the image plane is being projected onto the detector, while in momentum microscopy the back focal plane is imaged and in many cases, both types of imaging can be realised with the same instrument [252, 253].

The first HAXPEEM instrument began operation at beamline P09 at PETRAIII and was relocated to beamline P22 in 2018 [57, 254]. This microscope is based on a NanoESCA instrument (Scienta Omicron GmbH/FOCUS GmbH) modified to operate at higher electron energies [255]. The instrument uses an imaging double electron analyser (IDEA) with two hemispheres to compensate for aberrations [256]. Figure 11(b) shows first proof-of-principle energy filtered HAXPEEM images recorded of a calibration sample with gold checkerboard structure deposited on a silicon substrate at $6.5 \mathrm{keV}$. The intensity distribution of the $\mathrm{Au} 3 d_{5 / 2}$ 
(a)

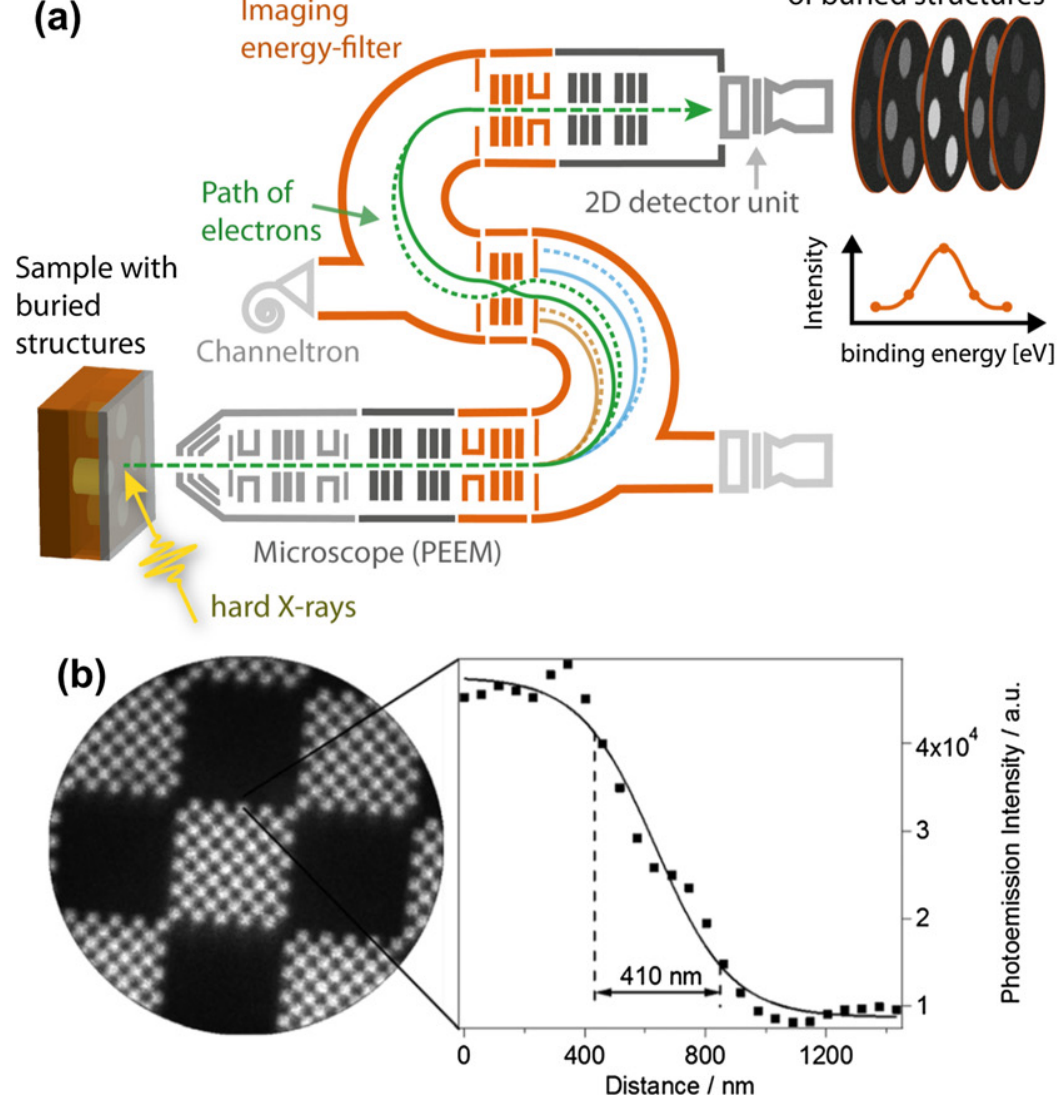

Image Spectrum

of buried structures
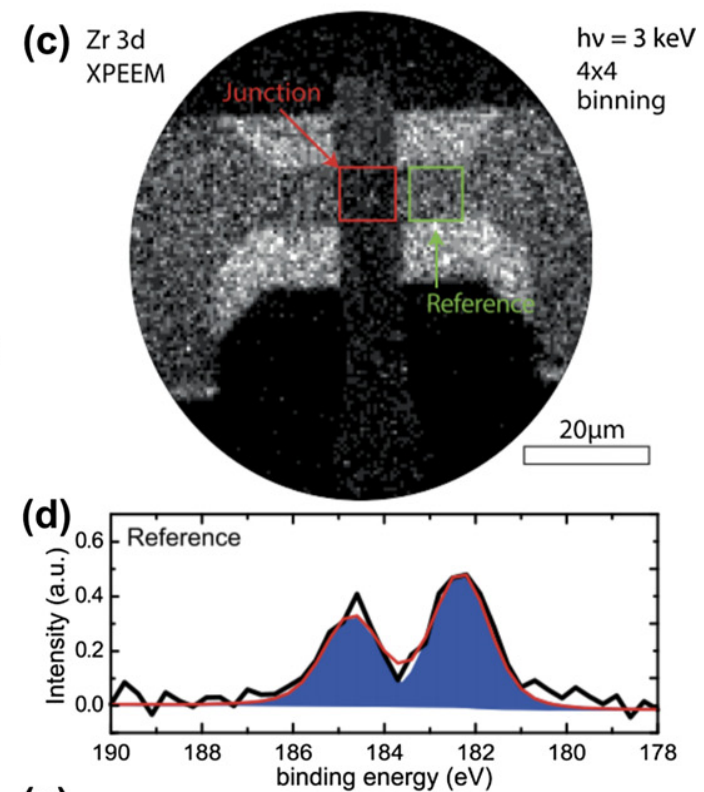

(e)

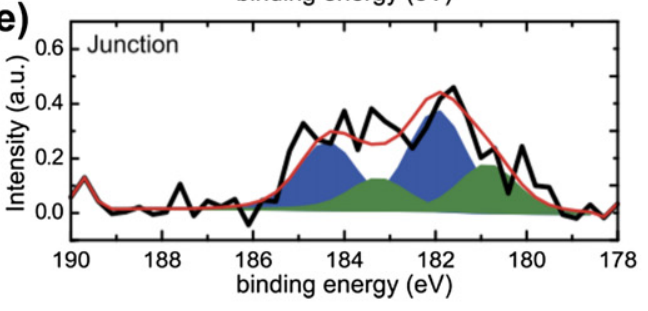

Figure 11. HAXPEEM. (a) The HAXPEEM analyser consist of a photoemission electron microscope (PEEM) optimised for high energetic electrons, an imaging energy-filter, and a 2D detector unit. Photoelectrons from buried layers are collected by the microscope, energy-filtered for a specific binding energy, and projected with a high magnification onto a $2 \mathrm{D}$ imaging unit. Thus, lateral resolved chemical maps can be acquired in one shot. By sweeping the filter-energy along the binding energy of a core-level, a lateral resolved image spectrum can be acquired. (a) Reproduced from [247]. CC BY 4.0. (b) First proof-of-principle results acquired from a reference sample for the Au $3 d_{5 / 2}$ photoemission line with a kinetic energy of $4.294 \mathrm{keV}$. The edge profile confirms a resolution of $410 \mathrm{~nm}$. (b) Reprinted from [248], with the permission of AIP Publishing. (c) HAXPEEM spectromicroscopic image of the distribution of the $\mathrm{Zr} 3 d$ intensity of a $\mathrm{ZrO}_{x} \mathrm{ReRAM}_{\text {device, }}$ the two marked areas are the active part of the junction where switching occurred (red) and a pristine reference part (green). The integrated spectra of the reference area (d) and the junction area (e) show additional components. (c)-(e) Reproduced from [249]. CC BY 4.0.

photoemission line with a kinetic energy of $4.294 \mathrm{keV}$ was recorded. A line profile at a sharp edge showed a spatial resolution of $410 \mathrm{~nm}$ [248]. Since these first proof of principle experiments, the instrument has shown its capabilities in several studies particularly for the imaging of micro-structured and electronic devices, as well as in corrosion science, which are summarised here.

While it is accepted that the switching mechanism in memristive ReRAM devices with a $\mathrm{ZrO}_{x}$ active layer, is based on the movement of oxygen vacancies resulting in a change of the valence state of the metal ions, very little experimental verification of the valence changes in such memristors have been reported. Kindsmüller et al used HAXPEEM to study such ReRAM devices with different metal top electrodes in high and low resistive states [249]. An HAXPEEM spectromicroscopic image of the distribution of the $\mathrm{Zr} 3 d$ intensity observed is shown in figure 11(c) and the corresponding spectral information of two regions is shown in (d) and (e) of the same figure. It can be seen that that zirconia in the active junction is reduced compared to a reference region due to the valence change in the $\mathrm{ZrO}_{x}$ film during electroforming. Further analysis showed a redox reaction at the $\mathrm{Ta} / \mathrm{ZrO}_{x}$ interface driven by oxygen movement in the oxide layers for different the resistive states. These results illustrate the unique insights HAXPEEM can offer in the characterisation of electronic devices.

An extension to the concepts of depth profiling by variation of energy and/or angle (as discussed in sections 3.1.1 and 3.1.5) is available through the analysis of the extended inelastic background based on Tougaard's formalism [257-259]. An advantage of this approach is that information from depth beyond the probing depth becomes available and the most obvious use of this technique is for studies of microstructured and layered samples. To showcase this, Renault et al acquired HAXPEEM spectromicroscopy images of the $120 \mathrm{eV}$ energy loss features in the Ti $1 s$ core level of a patterned epitaxial $\mathrm{Al}_{0.25} \mathrm{Ga}_{0.75} \mathrm{~N}$ channel and a Ti/Al metal contact with a $100 \mu \mathrm{m}$ field-of-view [260]. From the data the detailed Ti distribution below the channel at depths from 14 to $25 \mathrm{~nm}$ below the surface could be reconstructed. This shows how an entire $3 \mathrm{D}$ device stack can be imaged by hard x-ray spectromicroscopy. 
In contrast to the previous studies discussed, the final example focuses on recent work in the field of corrosion science [261]. In both air and aqueous environments most metals spontaneously form thin (1-3 nm thick) oxidic surface layers, which prevent further interactions with the underlying metal, and therefore defines the corrosion resistance of the material. Duplex stainless steels (DSS) have a high corrosion resistance despite their intricate heterogeneous microstructure of ferrite and austenite phases. There has been a debate whether the passive film on DSS is homogeneous. In a correlative approach using HAXPEEM and electron backscatter diffraction these surfaces could be analyzed on a grain by grain basis. The results showed small but detectable difference in the layer thickness $(2.1 \pm 0.4 \mathrm{~nm}$ ) and compositions (80 at.\% of Cr oxide) for ferrite and austenite grains, where ferrite grains contains more $\mathrm{Cr}$ oxide. On grain level the (111) oriented ferrite grains showed a lower content of $\mathrm{Cr}$ oxidic species in the outer layer.

To summarise, HAXPEEM combines sub-micrometer spatial resolution and a variable field-of-view with the high probing depth of HAXPES. Coupled with the analysis of the inelastic background, the probing depth can be extended to several tens on nanometres. Therefore, it is a very promising technique for the analysis of microstructured and layered samples in materials science and microelectronics. Ongoing developments in the field including new and upgraded instrumentation promise a wider application of the technique. A particularly interesting prospect is the use of different $\mathrm{X}$-ray polarisation regimes (see discussion in section 3.2.2) for HAXPEEM to allow magnetic grain imaging in buried structures. Furthermore, HAXPEEM can be combined with a $2 \mathrm{D}$ imaging spin-filter behind the double-hemispheres as well as with an additional ToF detector. The latter approach, referred to as xToF, uses the double-hemispherical analyser as a band pass filter to limit the electron energy distribution to an optimal energy window for the subsequent ToF energyfilter. Unwanted energies are cut-off and an overlap of spectra generated from subsequent pulses at high repetition rates are avoided as well as any unnecessary extra load on the delay-line-detector of the ToF energy-filter. This approach can increase the measurement efficiency of HAXPEEM by more than a factor of 10 using the typical high-pulse time structures of most synchrotrons.

3.1.7. Spin-resolved HAXPES. All transport and thermodynamic phenomena depend on topology of the Fermi surface, dispersion of the bulk bands, and density of states close to the Fermi edge $\left(E_{\mathrm{F}}\right)$. In order to elucidate the role of the electron spin in such phenomena, it seems highly attractive to record true bulk spin-polarisation spectra. Spin-resolved HAXPES could ensure that results are not corrupted by surface effects, which can drastically alter photoelectron spins. Moreover, detailed information on the true bulk electronic structure including the spin degree of freedom is mandatory for basic materials research and design.

Spin-resolved PES started in the 1970s and has become a widely used tool for electronic structure analysis. Comprehensive discussions of the field can be found in textbooks (see references [262-264]) and reviews (see references [265, 266]). Before turning to work in the x-ray range, it is important to recall the classification scheme leading to photoelectron spin polarisation. Early work focused on ferromagnetic systems [267], where the exchange interaction gives rise to a spin polarisation in the ground state. Spin-resolved bandmapping of ferromagnets [268] nowadays is a standard tool for electronic structure analysis. Fano's prediction of the optical spin orientation in free Cs atoms by circularly polarised light (Fano effect [269]) was transferred to solid-state photoemission [270] and launched the success story of the GaAs sources of spin-polarised electrons [271]. Spin polarisation arises as a consequence of spin-orbit interaction along with the relativistic dipole selection rules for optical transitions. Generalisation of Fano's mechanism to various non-magnetic solids constituted spin-resolved bandmapping of non-ferromagnets [272]. The paradigm that either ferromagnetism or circularly polarised light are mandatory for the occurrence of polarised photoelectrons was falsified by the discovery of highly spin-polarised photoelectrons released by linearly or even unpolarised light $[273,274]$. In this case, the spin quantisation direction points along the normal of the reaction plane formed by photon beam and outgoing electron. The symmetry properties are identical to spin-dependent Mott scattering. Finally, spin-momentum locking, a second type of groundstate polarisation has been predicted and verified for topological states $[275,276]$. Such states with complex 3D textures are intensely studied, because these special spin signatures are at the heart of research on topological and quantum materials [192, 277, 278].

In comparison with low-energy work, spin-resolved photoemission experiments in the x-ray range are sparse. Early core level measurements at ferromagnets used laboratory sources [279-281]. The feasibility of spin-HAXPES has been shown at several synchrotron endstations with different types of spin detectors, including P09 at PETRA III using a miniMott detector [254], and several SPring- 8 beamlines, including at BL47XU using a W(100)-type SPLEED detector [282], at BL15XU using forward Mott scattering in an Au film covering a FeNi sample [283], and at BL09XU using the same SPLEED detector [284]. In all four experiments, the spin-polarisation of the Fe $2 p$ core level spectrum was recorded and an example is shown in figure 12(a) (from [254]). The interplay of the $2 p$ fine-structure splitting and ferromagnetic exchange splitting leads to characteristic asymmetric spin profiles of the $2 p_{1 / 2}$ and $2 p_{3 / 2}$ lines. To our knowledge only a single experiment in the valence range has been reported so far, the result is shown in figures 12(b) and (c) (from [284]). This measurement revealed the spin profile across the valence band of an FeCo layer, a functional layer used in tunneling devices, buried beneath $3 \mathrm{~nm}$ of oxidic material. While this experiment clearly established the proof-of-principle of spin-HAXPES in the valence range, the spin-resolved signal was close to the detection limit.

The combination of low photoionisation cross sections at hard x-ray energies with the low efficiency of single-channel spin detectors is prohibitive for $k$-resolved spin measurements. A way out of this dilemma would be to exploit the efficiency 


\section{Exchange interaction}
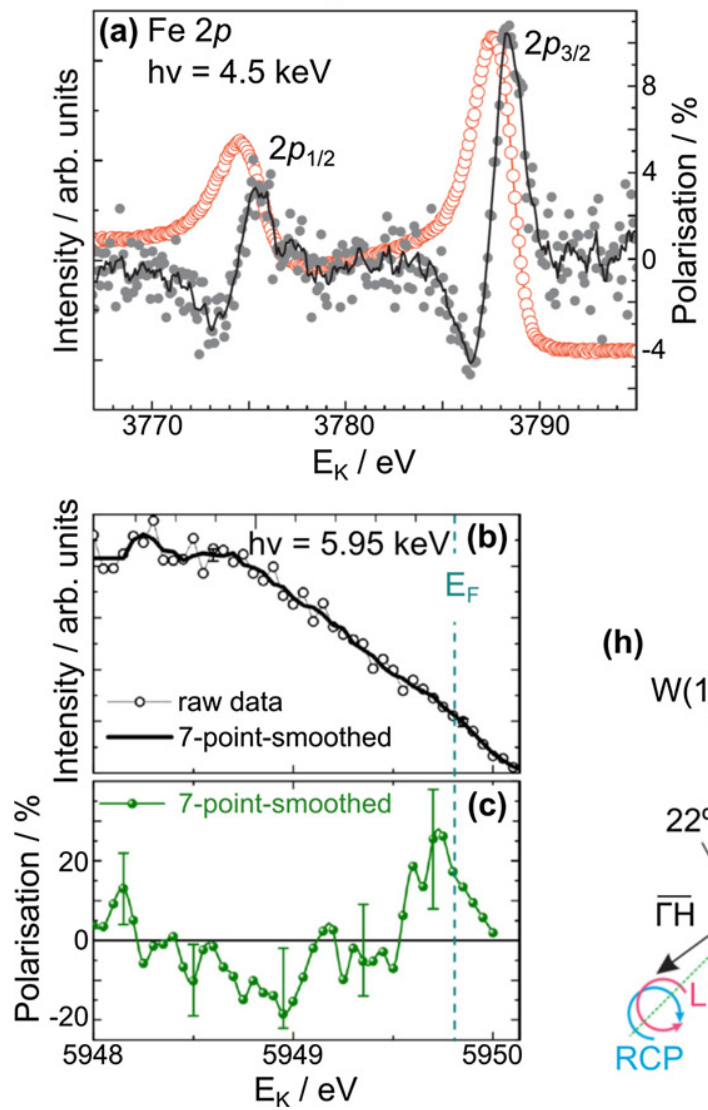

Spin-orbit interaction

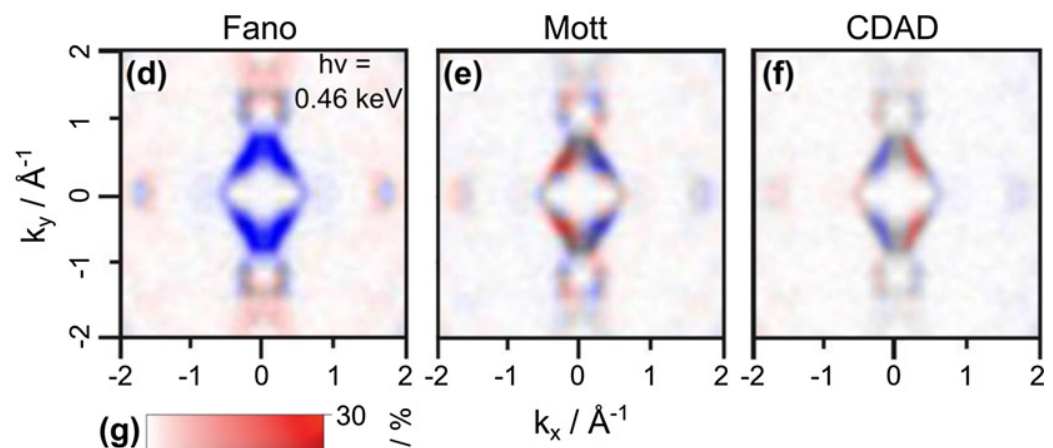

(h)

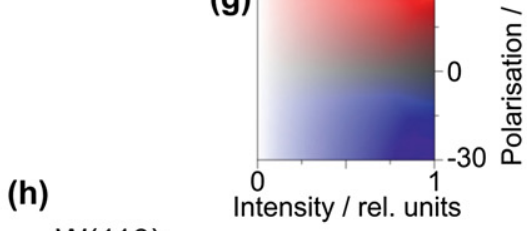

$\mathrm{W}(110)$

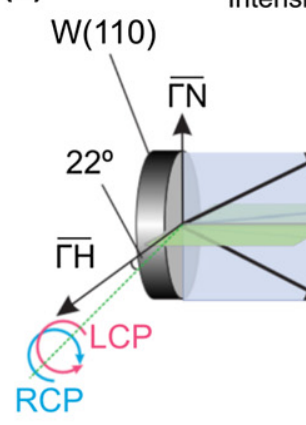

DLD2

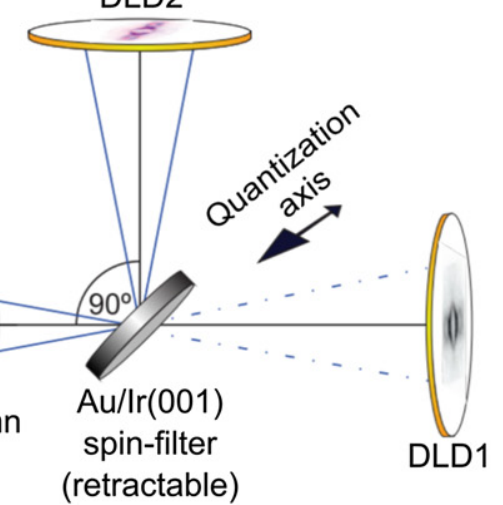

Figure 12. Overview of spin-HAXPES measurements in the hard (a)-(c) and soft x-ray range (d)-(h). The examples correspond to the three mechanisms that give rise to spin-polarised photoelectrons in bulk photoemission. (a) Spin profile of the Fe $2 p$ core-level doublet in a ferromagnetic FeCo film (Reprinted from [254], Copyright (2012), with permission from Elsevier) recorded with a mini-Mott detector. (b) and (c) Intensity and spin character of the valence bands of a FeCo film observed close to the Fermi energy (Reprinted from [284], Copyright (2016), with permission from Elsevier). In these ferromagnetic samples, the spin orientation is an initial state effect, originating from exchange interaction. (d) and (e) $k$-resolved spin-polarisation texture of the spin-orbit mediated Fano and Mott components in tungsten, recorded using circularly polarised soft x-rays. (f) Corresponding pattern of the circular dichroism CDAD. (g) 3D color scale with red, blue and gray denoting positive and negative spin polarisation and unpolarised intensity. (h) Experimental geometry. (d) - (h) Reproduced from [199]. ๑ IOP Publishing Ltd. All rights reserved.

gain of multichannel spin recording (see, e.g. figure 28 of [285]). Indeed, the first spin- and $k$-resolved experiment in the $\mathrm{X}$-ray range has been successful at the circularly polarised soft x-ray beamline P04 of PETRA III, using a ToF momentum microscope with imaging spin filter [286]. Similar experiments with a high-energy version of this type of microscope used hard X-rays from beamline P22 at PETRA III. Measurements for $\mathrm{EuO}, \mathrm{Fe}_{3} \mathrm{O}_{4}$ and the half-metallic ferromagnetic Heusler compound $\mathrm{Co}_{2} \mathrm{MnSi}$ (with protective capping layer against ambient air) have been successful. So far, no $k$-resolution could be demonstrated due to technical obstacles: fast electrons released by higher-order photons cause a strong secondary background on the spin-filter crystal (calling for a dispersive bandpass filter in the ToF column).

The measured full-field spin textures of W (figures 12(d) and (e)) have been recorded with circularly polarised x-rays of $460 \mathrm{eV}$. The retractable spin filter in the schematic setup (figure 12(h)) allows switching between spin-integral imaging (delay-line detector DLD1) and spin filtered imaging (DLD2), where it acts like a mirror with high spin selectivity. The spin-orbit interaction manifests itself in two ways: the Fano component $\boldsymbol{P}_{\text {Fano }}$ (figure 12(d)) is predominantly negative (blue) and is a consequence of optical spin orientation by right and left circularly polarised light (RCP, LCP). Its spin quantisation axis points along the helicity vector of the photon beam. The Mott component $\boldsymbol{P}_{\text {Mott }}$ (figure 12(e)) shows a more complex bipolar structure and is aligned perpendicular to the electron momentum. Its symmetry behavior is identical to the polarisation in spin-orbit (Mott) scattering, because photoemission can be considered as half a scattering process. The Feynman formalism uncovered a fundamental relation between the Mott and Fano components and the circular dichroism CDAD (figure 12(f)), for details, see references [197, 199].

3.1.8. Time-resolved HAXPES (trHAXPES). HAXPES is now a well-established bulk-sensitive technique but in recent years there has been an active drive to extend the techniques capability by accessing additional domains, in particular the time domain. The development of XFEL facilities has spear-headed the development of time-resolved HAXPES (trHAXPES) with their unprecedented capability of providing ultrashort 
$(<100 \mathrm{fs})$ pulses at high photon energies. Such pulses enable the study of ultrafast (sub-picosecond) transient electron dynamics with element specificity and chemical sensitivity. Coupling these pulses with high photon energies opens up the opportunity to conduct time-resolved measurements within the bulk of a material, useful for vertical heterostructures with buried interfaces.

Time-resolved XPS measurements in the extreme ultraviolet and soft $\mathrm{X}$-ray regime (trXPS or trPES) have been well established within the last decade with several studies primarily conducted at the FELs FLASH and LCLS [129, 130, 135-137, 287]. However, within the last six years groups primarily operating out of SACLA and SPring-8, have translated time-resolved measurements to HAXPES [131, 138, 139, 288, 289].

The most effective scheme for time-resolved photoemission measurements involves using two photon pulses, in what is known as a pump-probe scheme. For trHAXPES, a nearinfrared (NIR) pump pulse is typically used to excite the system and then the dynamics of this excitation is detected after a well-controlled time delay $(\Delta t)$ by photoelectrons emitted by a probe pulse in the hard x-ray regime. By capturing core-level spectra in response to the laser-driven excitation and monitoring changes to peak positions, line shapes and widths, the dynamical information can be obtained.

Vacuum space-charge effects (SCE) arising when using ultrashort pump and probe pulses at high intensity act as the main principle bottleneck of these measurements. SCEs can severely distort the photoelectron spectra in the form of both spectral shift and spectral broadening, affecting the accuracy of the spectra. For this reason, addressing the SCEs was a key challenge for the first documented measurements [139, 288]. SCEs occur due to the Coloumb interaction between outgoing electrons and are inevitable when more than one electron is emitted due to the pump and/or probe pulse. In practical measurements, the number (more precisely, the linear spatial density) of photoelectrons per pulse has to be kept below a certain value compatible with the desired effective spectral resolution. Thus, the repetition rate of the (probe) photon source becomes the main practical bottleneck of tr-HAXPES.

One of the first key preliminary studies into trHAXPES was put forward by Oloff et al with experiments conducted at beamline 3 (BL3) of the SACLA XFEL (SPring-8, Japan). This publication presented a series of systematic studies into the influence of vacuum SCEs on the $\mathrm{V} 1 s$ and Ti $1 s$ emission of $\mathrm{VO}_{2}$ and $\mathrm{SrTiO}_{3}$, respectively [139]. Measurements were conducted using a $1.55 \mathrm{eV}$ NIR pump pulse with a $100 \mathrm{fs}$ pulse duration and an ultrashort (30 fs) probe pulse excitation energy of $8 \mathrm{keV}$ with a $20 \mathrm{~Hz}$ repetition rate and. The two beams hit the sample quasi-collinearly with an approximate $1^{\circ}$ separation. The overlap between the pulses is measured using a fast photodiode, with the time-delay between the pulses controlled with femtosecond resolution. Peak position shifts and broadening were extracted from the data using peak fit analysis. Results showed that as the pulse energy increased, significant broadening of the spectra and distinct positive shift in kinetic energy was observed. However, in comparison to the intrinsic lifetime width of the respective spectra, the additional broadening due to vacuum SCE could be kept smaller. This demonstrated that XFEL-based HAXPES is viable under the conditions tested and SCE can be minimised depending on the operating conditions (e.g. pulse intensity, spot size etc). Furthermore, experimental data was successfully compared to the results of $\mathrm{N}$-body numerical simulations and mean-field models. A theoretical approach was taken to model the probe pulse-induced vacuum space-charge effects, which can aid with the deconvolution of these effects from the temporal dynamics on the final spectra. Good agreement both qualitatively and quantitatively was shown between the experimental and theoretical results, which showed that this approach could be used to improve the practicality of future experiments. In the same year, Oura et al conducted a similar experiment studying the spectral shift of the Ti $1 s$ core level of $\mathrm{SrTiO}_{3}$ but also reported preliminary results on the study of the photo-induced valence transitions within $\mathrm{YbInCu}_{4}$ using trHAXPES with a synchrotron source, rather than XFEL. Additionally, the same methodology was also later used to study the intrinsic charge-carrier recombination dynamics in $\mathrm{La} \mathrm{SrTiO}_{3}$ [131]. SCEs were successfully reproduced using a simple analytical model, while the effects were significantly reduced to begin with by operating with a lower photon incidence angle.

Since these pioneering studies, Oura et al have applied trHAXPES at the $27 \mathrm{~m}$-long undulator BL19LXU beamline of SPring-8 with the aid of an X-ray chopper [289]. Despite the inferior time resolution (picosecond rather than femtosecond) and pulse energies provided at SPring-8 compared to SACLA, the synchrotron radiation (SR) pulses offer the opportunity to conduct similar pump-probe experiments with acceptable counting efficiency while reducing the impact from the dreaded SCEs. Proof of principle measurements were conducted by measuring the temporal evolution of the $\mathrm{Si} 1 \mathrm{~s}$ core level from $\mathrm{SiO}_{2} / \mathrm{Si}$ structures over a range of different pulse fluences and delay times. The aim was to study the surface space-charge dynamics and surface recombination effects during the photoemission process. The Si $1 s$ peak BE position was shown to shift first in the positive direction and then relaxing back toward the negative, on the nano-second timescale. These shifts were assumed to reflect the creation of high-density holes created during the photoemission process, followed by band bending effects and electron-hole recombination. The use of hard x-rays enabled the study of photovoltage effects within the bulk of the structure and at buried interface, overcoming the main constraint of surface-sensitive trXPS, providing much needed additional information which can improve the understanding of charge-transfer dynamics in critical energy harnessing systems/devices [134, 290-293].

Additional measurements to study the magnetisation dynamics of the spintronic system $\mathrm{La}_{1-x} \mathrm{Sr}_{x} \mathrm{MnO}_{3}$ (LSMO) thin film grown on $\mathrm{SrTiO}_{3}$ (STO). HAXPES measurements are able to resolve well-screened states of LSMO present as lower BE structures (LBES) in the Mn $2 p$ core level. The core level was measured during optical pumping, with the LBES fitted with a Gaussian curve to monitor the temporal evolution. A sudden collapse of the LBES was observed at the co-incidence of the two pulses and decayed further with time. This observation was assumed to indicate that the slow magnetic dynamics 
of the compound directly delays the electronic phase transition within the material [128]. These measurements successfully confirmed that trHAXPES can be achieved using synchrotron sources within the picosecond-sub-nanoscale timescale, offering an alternative to the XFEL experiment but with lower time resolution.

Ultrafast magnetisation dynamics in ferro-, ferri- and antiferromagnetic materials are of high current interest both from an application point of view for new switching paradigms in devices and from a fundamental science point of view, where understanding the driving forces of remagnetisation processes is crucial. Only in special cases like the metamagnetic phase transition of FeRh [294], can magnetic information be gained from pure intensity spectroscopy; in general, magnetic contrast is needed. Direct spin contrast via spin-resolved observation would be favourable, however, the intensity loss by the spin filter makes such experiments very demanding. A much simpler way is to exploit the MCDAD (magnetic circular dichroism in the angular distribution of photoelectrons) as an ultrafast element-specific magnetometer. Unlike the magnetooptical Kerr effect, which integrated over all contributing optical transitions, the MCDAD-method can probe a selected atom species in a buried layer of a device. MCDAD with hard x-rays was pioneered in static experiments by Kozina et al [295]. The increasing availability of circular polarisation at HAXPES beamlines (see table 2 in the supplementary information), is an encouraging development for the study of magnetic materials. Furthermore, tr-MCDAD offers access to sublattice dynamics in compounds, an important issue for understanding fast magnetic processes.

The future of trHAXPES appears promising as new high-repetition-rate XFELs are coming online and first trial experiments have successfully addressed the SCE issue through optimisation using both a practical design of experiment approach and theoretical models. The further development of trHAXPES will surely benefit from developments in instrumentation, namely the ToF momentum microscope, which is discussed in detail in section 3.1.3 [137].

\subsection{Excitation schemes}

3.2.1. X-ray standing waves (XSW). As discussed extensively in previous chapters, hard X-ray excited photoelectron spectroscopy provides dramatically larger probing information depth comparing to its soft x-ray counterpart due to the much higher kinetic energy of excited photoelectrons. As a caveat to 'seeing deeper', the depth selectivity is steered away from surface sensitivity to being depth-averaged across several nanometres. One of the ways to enhance depth specificity, for example to enhance signal from buried interfaces, is to employ so-called x-ray standing wave (x-ray SW, or XSW) excitation to alter the form of the $\mathrm{x}$-ray wavefield inside (and above) the sample. The technique, which combines XSW with X-ray spectroscopy, was first used by Batterman and his team using a laboratory x-ray source [296]. Developments of much brighter and well-collimated beams generated by synchrotrons in the late 1980s provided a much needed boost for the wider spread of this technique [297, 298]. Nowadays, several synchrotron facilities host HAXPES endstations capable of performing XSW photoemission experiments, with the overview of HAXPES beamlines shown in the table 1.

For practical reasons, the interference between the incoming and reflected X-ray wave is used as means of standing wave generation. Two different modes of XSW creation are commonly used: (1) Bragg reflection from a periodic layered structure (either single-crystal atomic planes or artificial multi-layers), or (2) near total reflection from planar samples. Assuming the reflection geometry (see figure 13(a)), the relationship between the light wavelength, incidence (and reflection) angle, and the period of the standing wave can be written as follows:

$$
\lambda=2 D_{\mathrm{SW}} \sin \left(\dot{\theta}_{\text {inc }}\right),
$$

where $\lambda$ is the $\mathrm{x}$-ray wavelength, $D_{\mathrm{SW}}$ is the period of the standing wave, and $\theta_{\text {inc }}$ is the incidence angle measured from the sample surface. One cannot miss the obvious resemblance of equation (2) with Bragg's law. Indeed, if one takes into account the Bragg reflection geometry, following Bragg's law $n \lambda=2 d \sin \left(\theta_{\text {inc }}\right)$, the period of the generated standing wave equals the periodicity of the $n$th order periodical spacing of the sample. Further details on the mathematical derivation of intensity modulations and other key parameters can be found in a book chapter written recently by Zegenhagen [299].

The electric field strength, or in other words the x-ray wavefield intensity, as a function of depth and incidence angle for a specific case of a multilayer is shown in figure 13(b). Both near total reflection and Bragg modes of XSW generation are covered in the simulation. The 1st order Bragg reflection region (at around $1.4^{\circ}$ ) exhibits wavefield modulation with the periodicity equal to the internal periodic structure of the sample, while the near total reflection region around the critical angle shows the standing wave generated above the reflecting interface with much larger period (well above $10 \mathrm{~nm}$ ).

Thanks to much shorter wavelengths used in HAXPES in comparison to conventional soft x-ray photoemission, Bragg reflection off the crystal atomic planes is widely used as means for standing-wave production. A large portion of SWHAXPES studies focuses on providing a detailed surface chemical and structural analysis with approximately picometre resolution. In the last decade, SW-HAXPES contributed to the fast growing field of 2D materials, revealing structure and interaction between these fascinating materials and their supports, adsorbents, and intercalants. Recent examples involve graphene prepared on $\mathrm{SiC}(0001)$ [300, 301], graphene transferred onto $\mathrm{Al}_{2} \mathrm{O}_{3}$ [302], and hBN systems on $\operatorname{Ir}(111)$ [303] and $\mathrm{Cu}(111)$ studied by two independent groups [304, 305].

Matching the period of the standing waves to the crystal atomic planes also allows experimental extraction of site- and consequently element-specific electronic valence band structure. Early works by Woicik et al demonstrated the approach in the case of GaAs, InP and $\mathrm{NiO}$ single-crystals [306]. The technique was later applied to a variety of functional materials, including hematite [307], the gap insulator $\mathrm{SrTiO}_{3}$ [308], and high $T_{\mathrm{c}}$ superconductor $\mathrm{YBa}_{2} \mathrm{Cu}_{3} \mathrm{O}_{7-\delta}$ [303]. Only recently, electron momentum resolution was added to this method, 

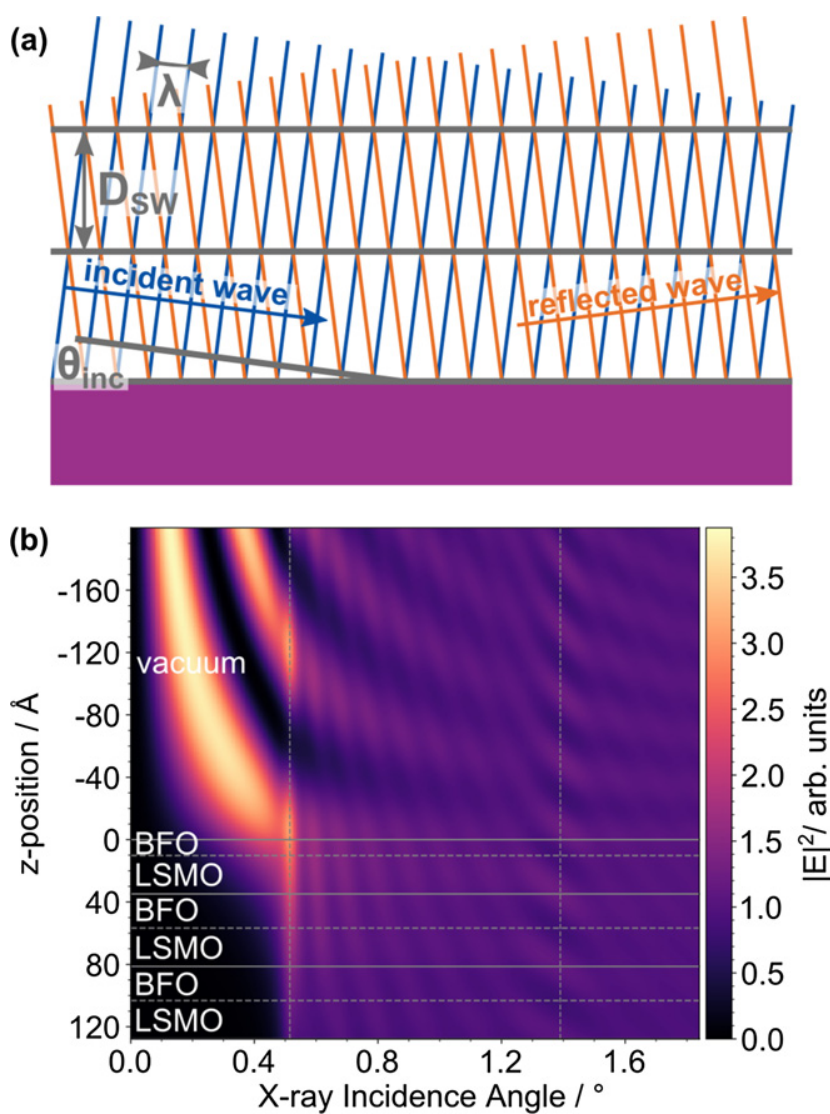

Figure 13. Principles of XSW. (a) Sketch of the interference pattern created by incident and reflected waves. All relevant geometrical parameters are marked. (b) X-ray electric field strength as a function of incidence angle and $z$-position with respect to the sample surface. Near total reflection region (incidence angle $0^{\circ}-0.4^{\circ}$ ) exhibits a standing-wave with 'long' period of $120 \AA$ (and more) above the sample and 1st order Bragg region (around $1.4^{\circ}$ ) shows the standing-wave in and above the sample with period equal to the period of the BFO/LSMO superlattice (nominally $48 \AA$ ).

demonstrating the technique on the prototypical dilute magnetic semiconductor $\mathrm{Ga}(\mathrm{Mn}) \mathrm{As}$ [194]. Figure 14(a) shows hard x-ray angle-resolved data measured at $\approx 3 \mathrm{keV}$ excitation energy. Using SW intensity modulations of Ga and As core levels, the valence band data were projected into $\mathrm{Ga}$ (including Mn dopant, which occupies substitutional Ga sites) and As contributions (see figure 14(b)). Theoretical calculations in form of Bloch spectra projections (see figure 14(c)) show remarkable agreement with the experimental decomposition.

Turning attention to SW-HAXPES studies of synthetic oxide multilayers, the first proof-of-principle study was carried out by Gray et al at beamline BL15XU at SPring-8 [309]. For this combined hard- and soft X-ray photoemission study of the interface between $\mathrm{La}_{0.7} \mathrm{Sr}_{0.3} \mathrm{MnO}_{3}$ and $\mathrm{SrTiO}_{3}$, an epitaxial superlattice consisting of 48 bilayers of $\mathrm{La}_{0.7} \mathrm{Sr}_{0.3} \mathrm{MnO}_{3}$ and $\mathrm{SrTiO}_{3}$, each nominally four unit cells thick $(\approx 15.5 \AA)$, was synthesised on a $\mathrm{SrTiO}_{3}$ substrate. Both datasets were fitted simultaneously using the x-ray optical theoretical code developed by Yang et al [310], in order to obtain an unambiguous self-consistent model of this prototypical magnetictunnel junction (MTJ) interface. Around the same time,
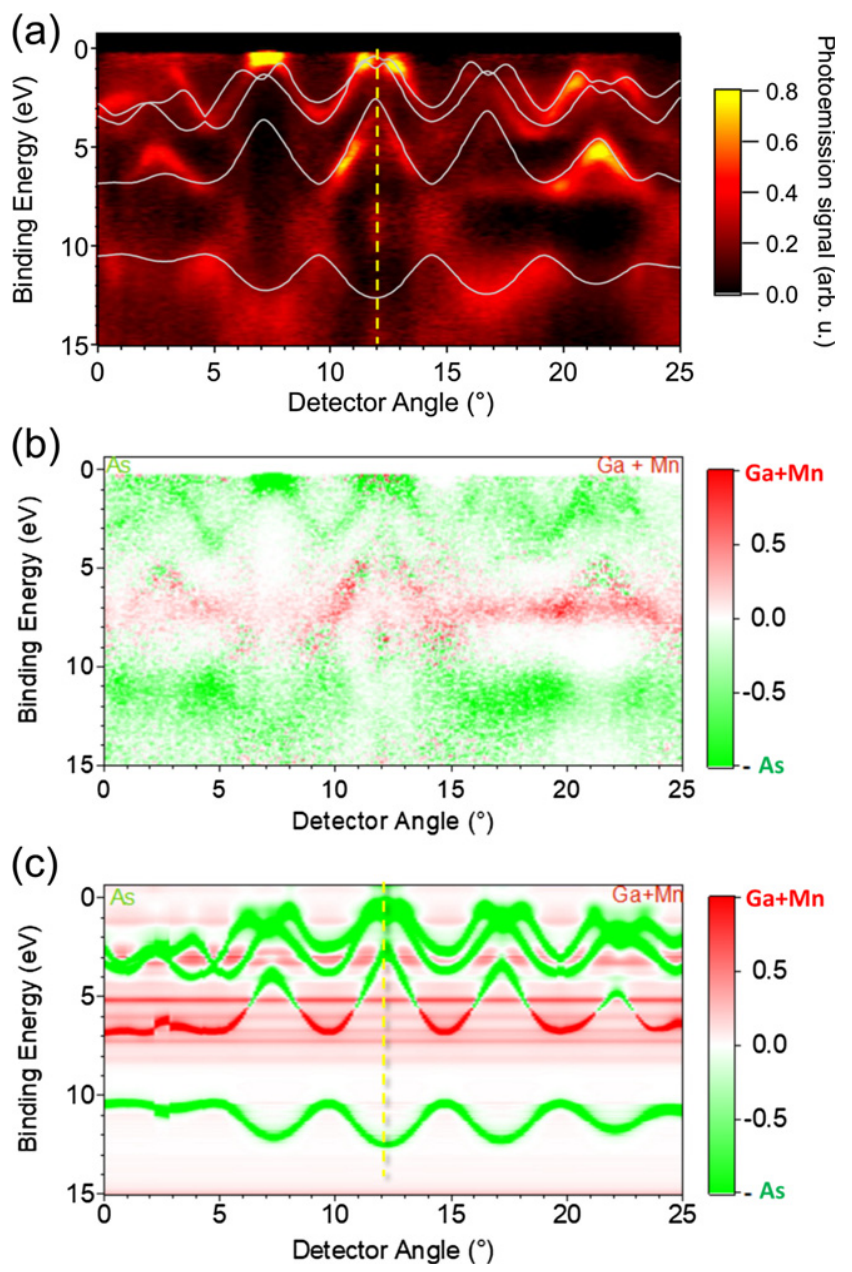

Figure 14. XSW and electron momentum resolution. (a) Normalised angle-resolved valence band photoemission data for $\mathrm{Ga}(\mathrm{Mn})$ As sample. (b) Decomposition of the data into $\mathrm{Ga}+\mathrm{Mn}$ and As contributions. (c) Bloch spectral function projection onto $\mathrm{Ga}+\mathrm{Mn}$ and As sites. Panels (b) and (c) show remarkable agreement. Reproduced from [194]. CC BY 4.0.

Döring et al applied SW-HAXPES for the investigation of a different functional interface relevant to spintronic applications-Fe/MgO [311], also later studied by SW-HAXPES by Conlon et al [312] More recently, Greer et al have utilised SWHAXPES to investigate the chemical interdiffusion of boron in a $\mathrm{Ta} / \mathrm{Co}_{0.2} \mathrm{Fe}_{0.6} \mathrm{~B}_{0.2} / \mathrm{MgO}$ trilayer, which is relevant to MTJs with perpendicular magnetic anisotropy [313].

Another field that has benefited from SW-HAXPES technique on multilayer samples is chemistry and electrochemistry of solid/liquid interfaces, using virtues of ambient pressure $\mathrm{x}$ ray photoelectron spectroscopy. The standing-wave ambient pressure photoemission spectroscopy (SWAPPS) technique was developed using soft x-ray excitation at the Advanced Light Source in 2014 and was applied to study adsorption of alkali ions onto hematite surface in aqueous solution [314]. Later, application to a corrosion study of nickel using hard $\mathrm{x}$ rays studied the buried interface through much thicker liquid film [315].

Lastly, several studies have utilised the near total reflection regime to generate SW modulation over the past few years. 
Marinova et al quantitatively measured polarisation-induced charge density changes at the interface between the ferroelectric $\mathrm{BiFeO}_{3}$ and the doped Mott insulator $\mathrm{Ca}_{1-x} \mathrm{Ce}_{x} \mathrm{MnO}_{3}$ [316]. Most recently, a combination of near total reflection and Bragg reflection approaches in SW-HAXPES were applied to hybrid multiferroic system of $\mathrm{BiFeO}_{3}$ and $\mathrm{LaSrMnO}_{3}$, revealing the nature of the electronic coupling between these two complex oxides [317].

SW-HAXPES is one of the few techniques that can provide full chemical and electronic information of a system together with spatial information on the atomic scale. With the steady improvement of light sources, electron energy analysers, and detectors, SW-HAXPES has a lot to offer in the near and distant future.

\subsubsection{Polarisation-dependent HAXPES. Photoelectron} spectra excited using hard $x$-rays show a pronounced dependence on the polarisation of the photon source. For linearly polarised light there are two cases that can be exploited in HAXPES experiments: vertically and horizontally, or $s$ (for German senkrecht) and $p$ (for German parallel), polarised light. As depicted in figure 15, in the case of vertically $(s)$ polarised light the polarisation direction is perpendicular to the plane between the photon and electron direction, while for horizontally $(p)$ polarised light the polarisation direction lies in the plane between the photon and electron direction. In the case of circularly polarised light the polarisation direction rotates at a constant rate in the plane perpendicular to the photon direction. It is important to note, that in most cases we cannot obtain totally polarised light and the degree of linear $P_{\mathrm{L}}$ or circular $P_{\mathrm{C}}$ polarisation, where 0 is unpolarised light and 1 is totally polarised light, is an important factor to take into account in polarisation-dependent experiments.

The dependence of HAXPES measurements on the polarisation of light in relationship to the photoemission geometry can be exploited to give a powerful tool to aid the identification of the specific orbital nature of contributions to valence states of materials. This technique is generally referred to as polarisation-dependent HAXPES and sometimes as linear dichroism in HAXPES. There are two different strategies to achieve polarisation-dependent HAXPES: (1) changing the polarisation of light with a phase retarder and postmonochromators, or (2) changing the analyser position with respect to the incoming X-rays. The first option is most common, while the second option is only implemented at BL12XU at SPring-8 [318-320]. The majority of HAXPES beamlines only deliver linear (horizontal) polarised light, with a small number of beamlines, including BL09XU, BL15XU, BL19LXU at SPring-8, GALAXIES at SOLEIL, and P22 at PETRAIII, being able to deliver both horizontal and vertical linear and even circular polarisation. A complete overview of polarisation modes available at beamlines worldwide is given in the supplementary information.

In parallel to experimental efforts, an important contribution to the application of polarisation-dependent HAXPES has come from Trzhaskovskaya and co-workers in the form of theoretical calculations of the parameters of the photoelectron angular distribution [78, 79, 321-323]. The landmark papers by this group are contributing significantly to our ability to understand and apply this technique to both valence and core states. The theoretical foundation for our understanding of the angular distribution of photoelectrons was laid by J W Cooper and others at the beginning of the 1990s [324]. This early work led to the establishment of the following equation:

$$
\frac{\mathrm{d} \sigma_{i}}{\mathrm{~d} \Omega}=\frac{\sigma_{i}}{4 \pi}\left[1-\frac{\beta}{2} P_{2}(\cos \theta)+\left(\frac{\gamma}{2} \sin ^{2} \theta+\delta\right) \cos \theta\right],
$$

where $\frac{\mathrm{d} \sigma_{i}}{\mathrm{~d} \Omega}$ is the differential photoionisation cross section for the $i$ th atomic subshell, $\sigma_{i}$ is the total photoionisation cross section for the same $i$ th atomic subshell, $\beta$ is a parameter describing dipolar effects, $P_{2}$ is the 2 nd Legendre polynomial, $\theta$ is the angle between the electron and photon directions, and $\gamma$ and $\delta$ are parameters describing non-dipolar effects. Both the photoionisation cross sections as well as the $\beta, \gamma$, and $\delta$ parameters depend on the $\mathrm{x}$-ray energy and therefore, calculated angular distributions, as shown in figure 16(a) for $\mathrm{Si}$ $1 s$ photoelectrons at energies between 5 and $11 \mathrm{keV}$, show a clear difference in the positions of the maxima with energy. Of course, there are also clear differences in the maxima with atom and atomic subshells across different photon energies as illustrated in figure 16(b).

The first report of a polarisation dependent HAXPES experiment was presented by Sekiyama et al in 2010 at beamline BL19LXU at SPring-8, which explored both core level and valence spectra of gold examining the intensity ratios of different orbital contributions [325]. In this initial experiment polarisation was achieved with a (100) diamond as a phase retarder in the Laue geometry with the 220 reflection obtaining $P_{\mathrm{L}}$ of -0.8 at $7.94 \mathrm{keV}$. Already in this early work the level of polarisation achieved was identified as an important parameter, and a few years later, the $P_{\mathrm{L}}$ at BL19LXU was further improved to -0.94 using again a (100) diamond but now in combination with a $\mathrm{Si}(620)$ postmonochromator [326]. Polarisation-dependent HAXPES can be very photon-hungry as phase retarders are in transmission mode and the additional channel-cut monochromator configurations lead to a loss in photon throughput. At BL19LXU this motivated further developments, including a phase retarder made of two single-crystalline (100) diamonds, improving the throughput of photons in the polarisation scheme significantly [327]. As mentioned above, while changing the polarisation of the incoming photons is the common approach to achieving polarisation-dependent HAXPES, an alternative approach was implemented at BL12XU at SPring-8 [318]. The HAXPES end station encompasses two hemispherical photoelectron analysers, which are mounted at two different angles to the incoming photon direction thereby changing $\theta$ in equation (3) and enabling polarisation-dependent HAXPES. The advantage of this approach is that the discussed losses which are usually encountered when phase retarders are used do not occur.

To date, polarisation-dependent HAXPES has been applied to a range of materials. Transition metal oxides have received much attention and several papers explore both their core level and valence band spectra $[318,327]$. Particularly the $3 d$ transition metals are a perfect application area for this technique, as 
(a)

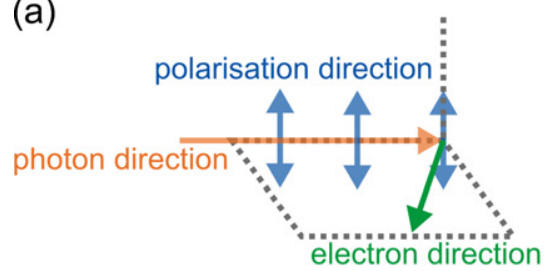

vertically linear polarised

s-polarisation (b)

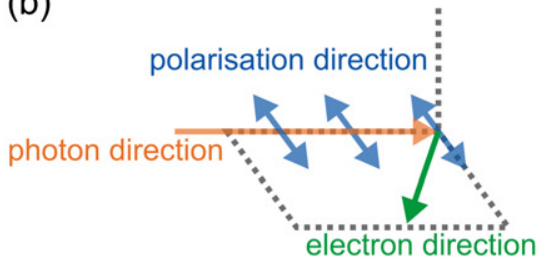

horizontally linear polarised p-polarisation (c)

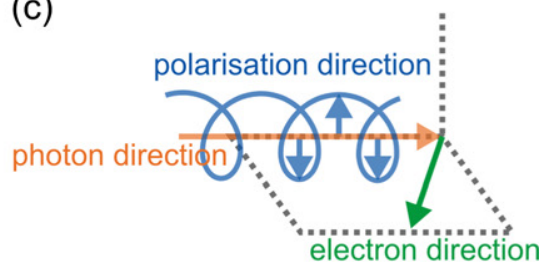

circularly polarised

Figure 15. Schematic of the polarisation of light in relationship to the photoemission geometry, including (a) vertically or senkrecht $(s)$ linear polarised light, (b) horizontally or parallel $(p)$ linear polarised light, and (c) circularly polarised light.
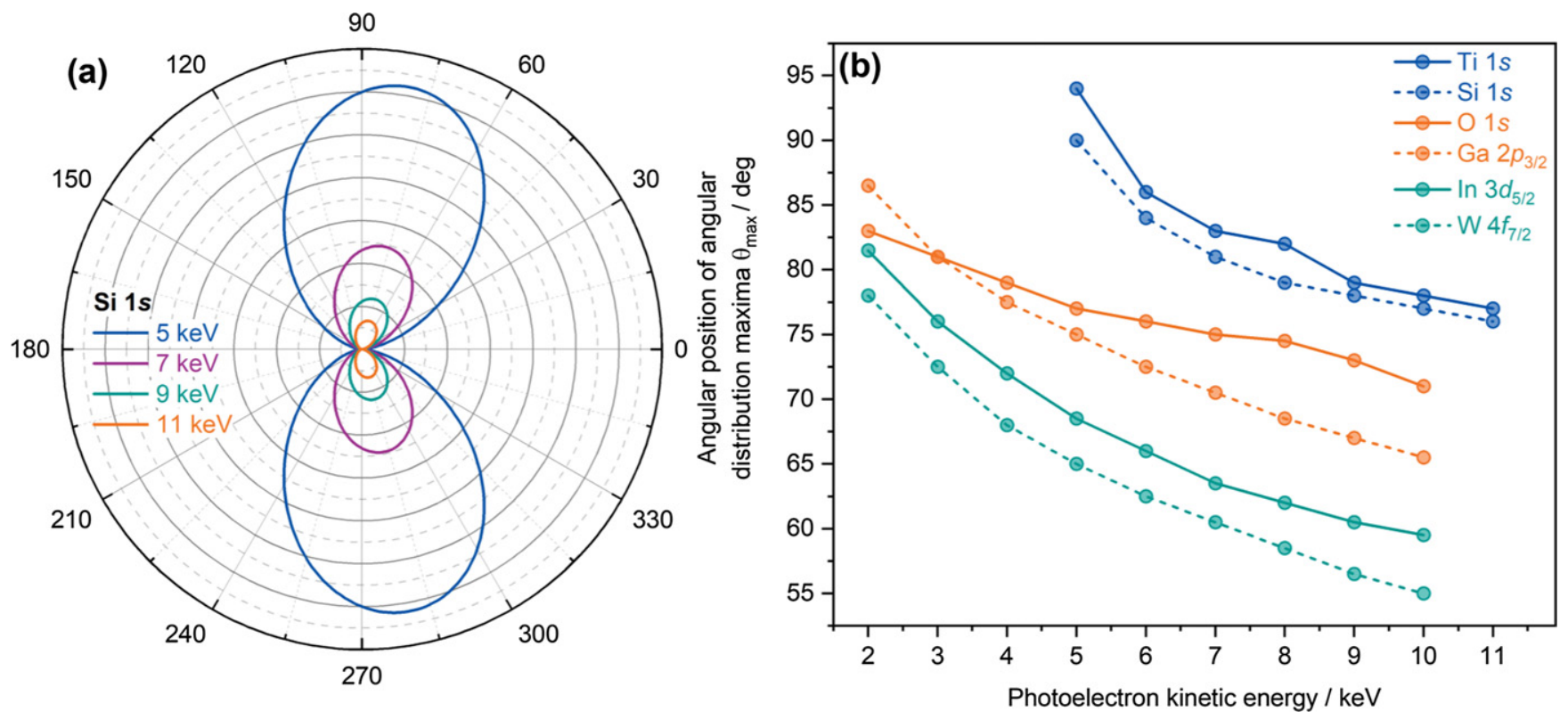

Figure 16. Angular asymmetry of photoelectrons. (a) Calculated angular distributions of $\mathrm{Si} 1 \mathrm{~s}$ photoelectrons between 5 and $11 \mathrm{keV}$. (b) Calculated positions of the angular distribution maxima for a range of elements and orbitals. Data from reference [78, 79] were used to calculate the differential photoionisation cross sections shown in these figures.

the dominance of the $3 d$ states makes it challenging to decouple the contributions from $4 s$ and $4 p$ states in standard HAXPES. The difference in angular dependence of the orbitals can be exploited and in combination with theoretical input from DFT a complete understanding of their electronic structure can be achieved [328]. Beyond bulk transition metals and their oxides, this technique is also well placed to study and explore the effects of dopants in these systems. This was shown by Regoutz et al at beamline BL12XU, where the influence of Sn doped into anatase $\mathrm{TiO}_{2}$ was explored. Even at a low doping level of $1 \% \mathrm{Sn}: \mathrm{TiO}_{2}$ the location of the $\mathrm{Sn}$ (and Ti) $s$ states as well as their hybridisation with oxygen states could be clearly pinpointed [319].

Beyond the first row transition metals, a number of other materials have been the subject of investigation using polarisation-dependent HAXPES, including electronic and magnetic materials with temperature-dependent behavior $[254,326,329]$. A landmark study on a wide range of Heusler compounds by Ouardi, Fecher and Felser in 2013 showed the many facets of polarisation-dependent HAXPES by collecting core and valence spectra of bulk and thin films samples not only at different polarisations but at different excitation energies and temperatures and in combination with theory [330].

The majority of studies discussed so far employs vertically and horizontally linear polarised light. A smaller number of studies has been able to use circularly polarised light and Ueda and Hamada give a comprehensive overview of the difference in the valence bands of $3 d$ transition metals when collected with linear or circular polarised light [328]. A particular advantage of the use of circular polarised light is in the study of the magnetic circular dichroism in materials $[243,295,331]$. The study on $\mathrm{CoFe}$ and $\mathrm{Co}_{2} \mathrm{FeAl}$ ferromagnetic films buried beneath $\mathrm{MgO}$ layers and grown on MnIr exchange-biased magnetic layers by ViolBarbosa et al combines polarisation-dependent HAXPES with angulardependent HAXPES enabling the exploration of the electronic states and the magnetic response from buried layers not only with polarisation, but also with location in depth [243]. This is an excellent example of how the combination of the 
different modalities of HAXPES can lead to even more powerful insights into structured samples.

As the number of beamlines that can deliver varying polarisation schemes increases, many more users will have the opportunity to apply polarisation-dependent HAXPES in all its variants to their materials and samples. A further possible future development is the use of vortex or twisted beams. While in circular polarised light the electromagnetic field vector rotates around the beam's central axis, in a vortex beam, often called an orbital angular momentum (OAM) beam, it is the phase of the light wave that rotates around the central axis. It traces out a helical path along the beam axis with the phase undefined along the axis, causing the beam to appear as a ring with a dark center. The topological charge $\pm l \hbar$ is a measure of the amount of OAM in a beam, where $\hbar$ is the fundamental quantum of angular momentum. The sign indicates the rotational direction, so whether the helical path is right or left handed. Vortex beams have recently been created at UV and soft x-ray energies [332, 333], but importantly for polarisation-dependent HAXPES, Seiboth et al recently reported the successful creation of hard x-ray beams with an OAM of topological charge $-\hbar$ and $-3 \hbar$ at a photon energy of $8.2 \mathrm{keV}$. This was achieved by using spiral phase plates made from fused silica [334]. While we may still have to wait some time for OAM-dependent HAXPES to become a reality, proof-of-principle studies observing an OAM-dependent dichroic photoelectric effect in He atoms are encouraging [335].

\subsection{Sample or sample environment}

3.3.1. Gas-phase HAXPES-HAXPES on dilute samples. The scientific interest in the interaction between isolated atoms and molecules with hard $\mathrm{x}$-rays is long standing and a comprehensive discussion is given in a chapter by Simon, Piancastelli, and Lindle in the 2016 HAXPES book edited by Woicik [48]. HAXPES on gas phase samples has a large and diversified interest, ranging from ultrafast nuclear motion occurring in the sub-fs time scale up to double-core-hole spectroscopy, postcollision interaction, recoil photoionisation studies, cascade Auger effects, and more. Here, selected examples of studies of fundamental interest in the gas phase are reported, which could also be extended to condensed phase experiments. Nowadays, gas phase HAXPES measurements are predominantly performed at SOLEIL and SPring-8 [336, 337]. End stations combine up-to-date hemispherical analysers with gas cells, which allows a high sample density (about two orders of magnitude higher than in the vacuum chamber) and a well-defined potential in the interaction region, crucial for high-resolution spectroscopy.

Double-core-hole (DCH) studies have received much attention as they can deliver a higher chemical sensitivity compared to traditional PES. This was predicted by Cederbaum in 1986 and finally experimentally observed in 2018 by Koulentianos in the acetonitrile molecule $\left(\mathrm{CH}_{3} \mathrm{CN}\right)[338,339]$. The creation of a DCH state implies the simultaneous ejection of two core electrons, however, the measurement of their kinetic energies with high resolution is a goal not yet reachable. Instead of creating doubly-charged DCH final states, with HAXPES a new strategy has been possible, namely to generate discrete DCH states for which one core electron is ejected into the continuum and the other core electron is promoted into an empty molecular orbital. In this case, only one electron has to be measured with high resolution. The proof of principle has been demonstrated by Puttner et al in argon by measuring $\operatorname{Ar}^{+}\left(1 s^{-1} 2 p^{-1} n l\right)$ Rydberg series converging to $\operatorname{Ar}^{2+}\left(1 s^{-1} 2 p^{-1}\right)$ DCH states [340]. Over the past few years, this technique has been successfully applied to a number of different atoms and molecules [341-345]. Another interest of DCH states is related to their highly dissociative character. Ultrafast dissociation (i.e. dissociation faster than the DCH lifetime) has been observed in the $\mathrm{HCl}$ molecule with two $2 p$ holes created after KLL Auger decay leading to the emission of an electron from the chlorine fragment [346, 347]. In case of the $\mathrm{H}_{2} \mathrm{O}$ molecule, ultrafast nuclear motion in the DCH of water has been observed even with a $\mathrm{DCH}$ lifetime of only 1.5 fs [348]. These observations have been obtained by measuring the Auger decay of these DCH states. The main Auger decay channel is a subsequent Auger decay, in which each decay step is filling one core hole with the ejection of two outer-shell electrons. The first Auger decay is called hypersatellite Auger decay. Another possible decay pathway of these $\mathrm{DCH}$ states is the two electron one electron (TEOE) process, which leads to the simultaneous filling of the two core holes and the emission of only one Auger electron, associated with the ejection of three electrons from the outer shells [349]. Note that the TEOE has a relative cross section of $1 \%$ in case of argon compared to the subsequent Auger decays. Direct derivation of the potential energy surfaces of DCH states has been realised thanks to RIXS and HAXPES measurements performed at the same photon energy [350]. DCH spectroscopy using HAXPES as described above has been so far limited to diluted species, but undoubtedly, if DCH studies could be extended into the condensed phase, they would provide valuable information.

Recoil effects caused by the high photon energies used in HAXPES, are starting to be exploited in experiment, particularly in the gas phase. At high photon energies above the ionisation threshold, fast electrons with high momenta are ejected into the continuum. Because of momentum conservation, such high-energy electrons leaving the atom or molecule lead to a large recoil of the remaining ion. The recoil energy is shared between translational, vibrational, and rotational recoil. In case of an isolated neon atom, the translational recoil has been measured up to $13 \mathrm{keV}$, well above the neon $1 s$ ionisation threshold. An Auger Doppler effect has been observed with blue- and redshifted Auger electrons due to this large recoil [351]. When the photoelectron is emitted in a direction parallel to a chemical bond, the departing electron causes vibrational excitation. This effect has been observed in the CO molecule [352]. If the electron is emitted perpendicular to the chemical bond, a fast photoelectron is inducing large rotational recoil. This has been observed in the $\mathrm{CO}$ molecule [353]. A rotation period of the molecule shorter than the core hole lifetime at a photon energy of $12 \mathrm{keV}$, well above the $\mathrm{C} 1 \mathrm{~s}$ ionisation threshold, has been 
proposed to interpret the measured asymmetric Auger Doppler profile. First examples of recoil observed in solids have been reported in graphite [179, 180, 354].

Just above a deep inner-shell ionisation threshold, postcollision interaction (PCI) plays an important role. This process takes place in the presence of a slow departing photoelectron and a fast Auger electron. The physical picture is the following: a fast Auger electron starts to move away from the doubly charged atomic ion and passes the slow photoelectron. The photoelectron is then partially screening the Coulomb field felt by the Auger electron. The manifestation of PCI is a gain in kinetic energy by the Auger electron accompanied by a simultaneous loss of kinetic energy by the photoelectron. Just above the core hole level, the photoelectron can even be recaptured into an unoccupied Rydberg orbital. PCI also happens in the soft $\mathrm{X}$-ray domain, but is much stronger in the hard x-ray domain because the shorter core hole lifetimes and the faster Auger electrons. An illustration of these effects has been presented in case of argon KLL Auger just above the $\mathrm{K}$ shell [355], where shifted and distorted Auger lines above the K-edge, as well as $2 p^{-2} n p$ states, reached below threshold by resonant Auger decay, are present above the ionisation threshold. Differences in PCI effects on $\mathrm{Kr} \mathrm{L}_{3} \mathrm{M}_{4,5} \mathrm{M}_{4,5}$ Auger electron spectra were recently published [356]. The PCI Auger shift depends on whether the initial photoionisation occurs slightly above the $\mathrm{K}$ threshold or slightly above the $\mathrm{L}_{3}$ threshold. Above the $\mathrm{K}$ shell, $\mathrm{K} \alpha$ fluorescence emission followed by Auger processes due to the $\mathrm{L}_{3}$ core hole takes place decay. Above the $\mathrm{K}$ shell, the time delay due to fluorescence causes a reduced PCI Auger shift, because the Auger electron overtaking the photoelectron happens later in time, compared to $\mathrm{L}_{3}$ shell photoionisation.

Ongoing instrument developments, including narrow photon bandwidth options e.g. at the GALAXIES beamline at SOLEIL based on a four-bounce high resolution monochromator [36], open up new avenues for high resolution gasphase studies, e.g. on recoil. Furthermore, the introduction of polarisation-dependence and the use of very high photon energies in the gas phase present attractive opportunities for new experiments. Beyond technological advances, moving from molecular gas-phase studies toward the exploration of gas phase clusters is an interesting prospect.

3.3.2. Ambient pressure HAXPES (AP-HAXPES). In situ and operando studies of surfaces and interfaces during catalytic and electrochemical processes are essential to address some of the most pressing challenges in energy storage and conversion. Solid/gas and solid/liquid interfaces are at the heart of many technologically important systems, with the first playing a big role in heterogeneous catalysis and the latter controlling all electrochemical reactions. For a technique that relies on ultra-high vacuum (UHV) environments to enable photoelectrons to travel from a sample surface through the analyser and to the detector where they can be counted, having gas or liquid phases in front of a solid catalyst or battery electrode surface is a tremendous challenge. Although ambient pressure environments were explored from the early days of Siegbahn's group in the 1970s [357, 358], it has been the advent of intense laboratory and synchrotron $\mathrm{x}$-ray sources in combination with new analyser designs and efficient detectors that has made ambient pressure experiments an established analytical technique [359-361].

The presence of gases and liquids seems at first to be incompatible with the UHV environments requires for photoelectron analysers and detectors. However, tremendous engineering efforts have established two practical solutions. Firstly, differentially pumped analysers can reduce the pressure applied at the sample (mbar to low bar) to the vacuum conditions required over comparatively short distances by using consecutive pumping stages. Secondly, cell designs that confine the gas or liquid behind a window (often silicon nitride or graphene) and therefore circumventing the need for differentially pumped systems can be used, however, the addition of the window further impedes photoelectron transmission. Beyond these technical, vacuum-related challenges the fundamental reduction in IMFPs when photoelectrons excited at a solid surface have to travel through a gas or liquid environment necessitate intense $\mathrm{X}$-ray sources and efficient detection schemes. The IMFP in 5 bar of $\mathrm{N}_{2}$ gas for example is $\sim 0.21 \mathrm{~mm}$ at $1.4 \mathrm{keV}$, with it depending strongly on the pressure and chosen gas environment [362]. Figure 17(a) illustrates the drop in signal intensity with increasing pressure in an APHAXPES experiment studying $\mathrm{Cu}$ in an He environment [363]. In liquids IMFPs are of course much shorter than in gases, with e.g. IMFP values of $\sim 10 \mathrm{~nm}$ in water at $5 \mathrm{keV}$ [156]. Currently, accessible gas pressure regimes (low mbar regime) and liquid overlayer thicknesses (a few 10s of nm) are strongly limited by these low IMFPs, which in turn limits their comparability to realistic and technological environments experienced by materials of interest under operation. The ultimate goals are to perform solid/gas experiments above atmospheric pressure at several bar and solid/liquid experiments with stable liquid overlayers of sufficient thickness to represent realistic conditions.

HAXPES can significantly aid in overcoming these limitation as the higher excitation energies lead to longer IMFPs. Depending on the gas pressure or liquid overlayer thickness an optimum excitation energy can be determined for a specific core level taking into account the decrease in photoionisation cross sections in the hard $\mathrm{x}$-ray regime. In view of this, figure 17 shows the dependence of the transmission through (b) water layers with thicknesses ranging from 10 to $100 \mu \mathrm{m}$ and (c) a layer of $\mathrm{CO}$ gas at pressures ranging from 0.1 to 2 bar as function of the photon energy. The normalised intensity $I\left(E_{\gamma}\right)$ detected for the $\mathrm{C} 1 s$ line from one monolayer at the solid/liquid interface after transmission through a water layer of thickness $d_{\text {water }}$ is given as

$$
I\left(E_{\gamma}\right) \propto \exp \left(-\frac{d_{\text {water }}}{\lambda_{\text {water }}}\right) \sigma(\text { C } 1 s),
$$

where $\lambda_{\text {water }}$ is obtained from reference [156], $\left.\sigma(\mathrm{C} 1 s)\right)$ is the photoionisation cross section of the $\mathrm{C} 1 s$ core level as function of energy [78]. The calculation shows a clear trade-off between the gain in transmission due to higher kinetic energy and loss in signal intensity due to dripping cross sections. For a water 

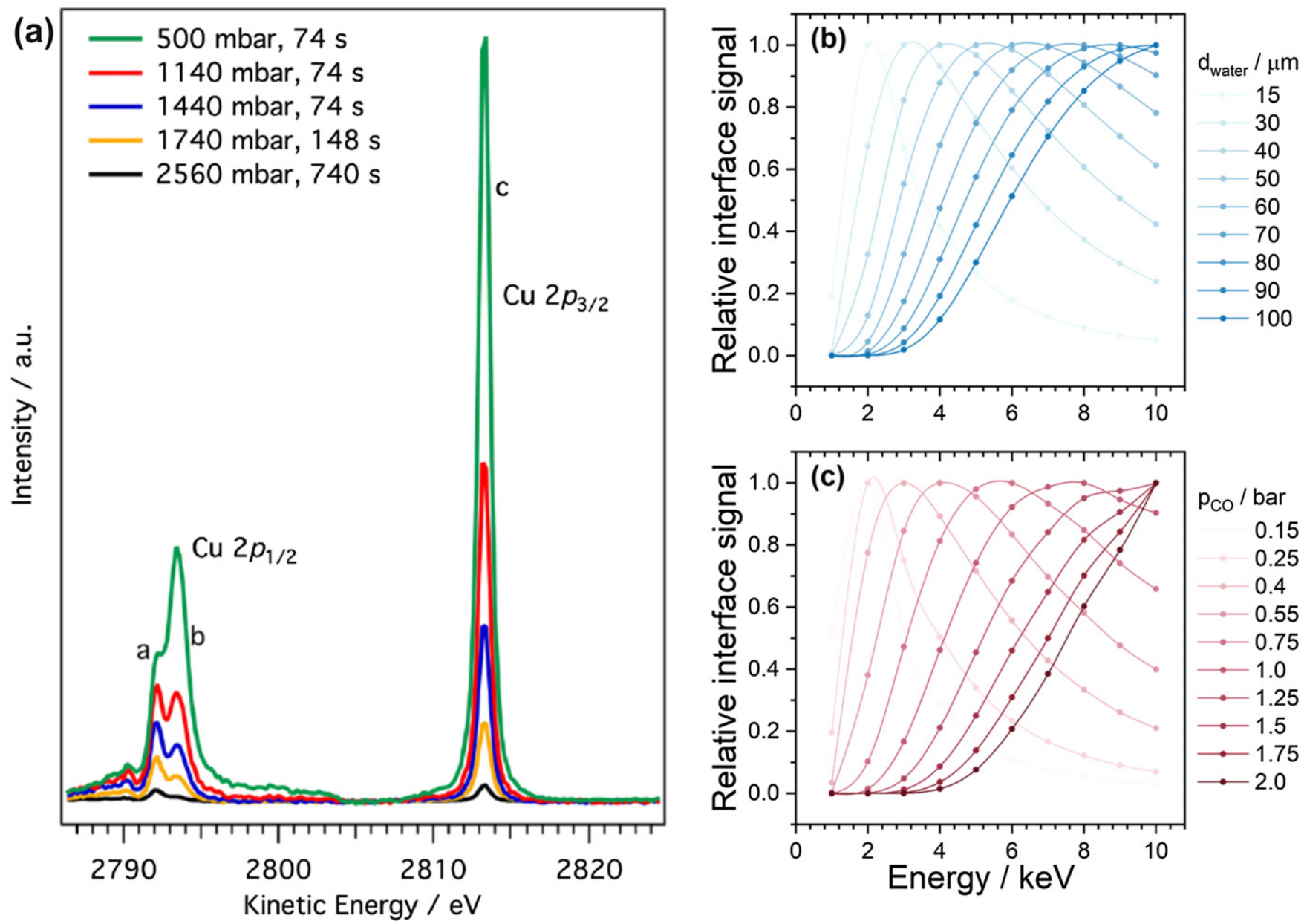

Figure 17. The dependence of signal intensity in AP-HAXPES. (a) $\mathrm{Cu} 2 p$ core level spectra of $\mathrm{Cu}$ taken at varying He pressures with an excitation energy of $3.75 \mathrm{keV}$ at beamline P22 at PETRA III. Reprinted from [363], with the permission of AIP Publishing. (b) and (c) The relative contribution of the $\mathrm{C} 1 s$ signal detected from a buried carbon monolayer as a function of photon energy, including (b) the signal detected under water layers with thicknesses ranging from 10 to $100 \mathrm{~nm}$, and (c) the signal detected at various pressures of CO gas at a constant sample to analyser distance of $20 \mu \mathrm{m}$.

layer with a given thickness of e.g. $50 \mu \mathrm{m}$ the strongest relative contribution from the interface is found for $4.5 \mathrm{keV}$ photons.

Moving on to the solid/gas interfaces, figure 17(c) shows the normalised intensity arriving at the detector under reaction conditions of $\mathrm{CO}$ gas at $T=230^{\circ} \mathrm{C}$ and at a given pressure $p$. Here, the size of the gas cushion (i.e. the distance to the analyser inlet) is set to $d_{\mathrm{VC}}=30 \mu \mathrm{m}$ and the sample (Fe, fcc) is in grazing incidence to enhance the surface signal. The data is obtained with the incidence angle set to half of the critical angle, $\alpha_{\mathrm{i}}=\alpha_{\mathrm{c}} / 2$, to ensure constant x-ray penetration $\left(\lambda_{\gamma}=2.1 \pm 0.05 \mathrm{~nm}\right)$. The reported intensity is that of $\mathrm{C} 1 \mathrm{~s}$ of the first mono layer calculated according to

$$
\begin{aligned}
I\left(E_{\gamma}, p\right) \propto & \exp \left(-\frac{d_{\mathrm{VC}}}{\lambda_{\text {gas }}}\right) \sigma(\mathrm{C} 1 s) \\
& \times \int_{0}^{d_{\mathrm{ML}}} \mathrm{d} z \exp \left(-z\left(\frac{1}{\lambda_{\mathrm{e}}}+\frac{1}{\lambda_{\gamma}}\right)\right),
\end{aligned}
$$

where $\lambda_{\text {gas }}$ is obtained from reference [364] for CO gas, $\lambda_{\mathrm{e}}$ is the IMFP of the electrons in the substrate as found in reference [156] and $\lambda_{\gamma}$ is the $\mathrm{x}$-ray penetration depth calculated from the Parrat equation [365]. Clearly the most important parameter in view of this is the transmission through the gas, as this can be changed by changing $p$ and $d_{\mathrm{VC}}$, while the other parameters are given by natural constants. Therefore, $d_{\mathrm{VC}}$ needs to be as small as physically possible. As expected, the intensity can be maximised by cleverly choosing the photon energy e.g. to about $4.6 \mathrm{keV}$ for $p=0.5$ bar. To reach higher pressures a higher $E_{\gamma}$ is required, showing that the balance of cross section vs transmission is the determining parameter in $I\left(E_{\gamma}, p\right)$.

Beyond the clear advantage of increasing IMFPs in APHAXPES, a recent study by Favaro et al discusses further advantages and limitations of AP-HAXPES from simulations of a model liquid/solid interface system [366]. At high photon energies secondary electron emission cross sections can be reduced and consequently limiting water radiolysis during experiments. Furthermore, the large discrepancies between hard x-ray energies and the energies of various molecular excitation modes and ionisation thresholds of typical gases used in ambient pressure experiments, are found to reduce photoelectron-gas inelastic scattering events. 
While soft x-ray based ambient pressure systems are well established in both laboratories and at synchrotrons, the number of hard x-ray systems is small [74, 359, 367-369]. Development of laboratory-based AP-HAXPES instruments started in the early 2010s by Kobayashi et al [74] Their system consisted of a monochromatic ULVAC PHI Cr K $\alpha$ x-ray source and a VG Scienta R4000 analyser. It was able to reach a maximum pressure of 10 mbar through the use of environmental cells. As discussed in section 2.2 new generations of laboratory systems are able to provide the high photon flux required to overcome both the low photoionisation cross sections as well as the lower count rates in ambient pressure experiments.

One of the first synchrotron-based AP-HAXPES endstations was established at beamline BL36XU at SPring-8 [370]. Initially, pressures could reach up to 40 mbar using a $20 \times 120 \mu \mathrm{m}^{2}$ beam size and a sample surface-aperture distance and standard electron analyser aperture of $300 \mu \mathrm{m}$. Further developments enabled the reduction of the x-ray spot size to $20 \times 20 \mu \mathrm{m}^{2}$ and the use of a working distance of only $60 \mu \mathrm{m}$ and an analyser aperture of $30 \mu \mathrm{m}$ resulting in the possibility to collect core level spectra in up to 1 bar of air [371, 372]. BL36XU has also been a trailblazer in combining ambient pressure environments with operando setups for fuel cells, which will be discussed in detail in section 3.3.3 [371, $373,374]$. Today, a number of AP-HAXPES beamlines is operational, including P22 (PETRA III), X07MB (SLS), and 9.3.1 (ALS) $[57,58,375]$. In addition, beamline $8 \mathrm{~A}$ at the Pohang Accelerator Laboratory, which is set up as a soft X-ray APHAXPES endstation, uses an off-line $\mathrm{Cr} \mathrm{K} \alpha$ source to perform AP-HAXPES [114].

The new approach of replacing standard environmental cells with a virtual cell design, as implemented at beamline P22 at PETRA III, can help to achieve pressures beyond 1 bar $[57,363]$. A virtual cell uses a gas outlet in the front cone of the electron analyser, such that a regional high-pressure environment of up to $10 \mathrm{bar}$ is generated at the sample surface while maintaining a low pressure of 5-10 mbar within the main chamber and pressure below $5 \times 10^{-6}$ mbar at the detector. The local gas flow creates a high-pressure gas pillow at the sample surface of only a few 10 s of $\mu$ m thickness [363]. The high-pressure environment is separated from the first differential pumping segment of the electron analyser via a $70 \mu \mathrm{m}$ thick Ti membrane, with several 10-30 $\mu$ m diameter apertures spread along the foot print of the photon beam to boost electron transmission. One advantage of a virtual cell approach is the ability to relatively quickly switch between different gases to study reaction dynamics.

Using grazing incidence geometry the surface signal is improved, important in studies of adsorbates on surfaces. Proof of principle measurements have been reported on a $\mathrm{Rh}(111)$ single crystal with a $\mathrm{CO}$ coverage at incidence angles below $\left(0.3^{\circ}\right)$ and above $\left(1.0^{\circ}\right)$ the critical angle of total reflection. The acquired $\mathrm{O} 1 \mathrm{~s}$ spectra show a factor of five increase in signal intensity due to the surface sensitivity of grazing angles. Experiments on $\mathrm{Cu}$ single crystals demonstrated the ability of the instrument to collect $\mathrm{Cu} 2 p$ core level spectra at pressures of up to 2.56 bar in $\mathrm{He}$ and 0.75 bar in $\mathrm{CO} / \mathrm{H}_{2}$ mixtures, both at room temperature. In both the $\mathrm{He}$ and $\mathrm{CO} / \mathrm{H}_{2}$ gas phase environments, electron energy loss features associated with the excitation of gas molecules are observed, which allows to follow reaction products. Temperature programmed experiments combined with a mass spectrometer were also explored with a view to incorporating these methods simultaneously in future XPS studies.

Beyond exploring solid/gas interfaces AP-HAXPES is a powerful tool for the exploration of solid/liquid interfaces. A well established technique to prepare thin liquid overlayers on solid substrates is the dip-and-pull method. This technique was initially explored by Axnanda et al at beamline 9.3.1 at the ALS, based on the works of Hansen and Kolb [376, 377], to create a thin, stable electrolyte layer on a Pt electrode [375]. Following developments at the APS, a setup for in situ electrochemistry measurements was developed at the tender x-ray beamline PHOENIX I at the Swiss Light Source, which is setup to study solid-liquid interfaces [58]. In first commissioning experiments a thin layer $0.1 \mathrm{M}$ of $\mathrm{KOH}$ aqueous electrolyte was prepared by dip-and-pull at 12 mbar with a $150 \mathrm{~nm}$ thick $\operatorname{Ir}(001)$ film as the working electrode. After cycles of electrochemical procedures during each dip stage, spectra were collected at the subsequent pull stages using an $\mathrm{x}$-ray photon energy of $4 \mathrm{keV}$, demonstrating the capability of preparing a stabilised ultra-thin electrolyte film on the Ir electrode for high pressure characterisation.

To gain insights into reaction kinetics, Nakamura et al introduced a time-resolved AP-HAXPES setup at BL36XU at SPring- 8 achieving a time resolution of approximately $0.2 \mathrm{~s}$ [374]. Typical pressures at the sample surface are reported to be approximately 0.1 bar with a maximum of 1 bar. One of the first experiments explored the redox processes at a $\mathrm{Pt}$ cathode during a sudden change in cathode-anode bias voltage. Using cyclical steps of the cathode-anode bias voltage as a time-trigger, spectra were accumulated to obtain reasonable signal-to-noise. The $\mathrm{Pt} 3 d_{5 / 2}$ core level spectra collected at varying bias voltages require large numbers of repetitions (up to 300) to achieve the necessary data quality for analysis and this approach is reminiscent of the cyclical processes explores in pump-probe time resolved HAXPES (see sections 2.3 and 3.1.8).

A major challenge in AP-HAXPES is the small signal intensity originating from the narrow interfacial region compared to that of the extended bulk liquid and solid layers. Based on previous experiments using soft x-rays [314], Karslioglu and co-workers addressed this issue by extending the experiment to standing wave ambient pressure photoemission spectroscopy (SWAPPS), allowing superior depth resolution in AP-HAXPES measurements [315]. First experiments on $8 \mathrm{~nm}$ polycrystalline $\mathrm{Ni}$ in $20 \mathrm{~nm}$ of $0.1 \mathrm{M} \mathrm{KOH}$ solution during oxidation were able to identify the complete oxidation/hydroxylation reaction of Ni under bias. Further details on the standing wave technique can be found in section 3.2.1.

The importance of AP-HAXPES to provide unique insights into solid/gas and solid/liquid interfaces thanks to the extended IMFP offered in the hard X-ray regime, as well as the possibility of conducting realistic experiments, is clear. AP-HAXPES has already proven invaluable across a number of scientific fields, most notably in heterogeneous catalysis and fuel 
cell development, where in situ or operando measurements can provide crucial information on reaction processes and material properties under realistic conditions. The future of AP-HAXPES looks to build on the many developments made over the last few years to address some of the current limitations of the technique. Efforts continue to achieve pressures surpassing 2.5 bar at the sample surface, to reach the realm of technologically important processes such as methanol synthesis at $\sim 10$ bar. One of the remaining challenges operando AP-HAXPES today is the long acquisition time, which consequently limits our ability to study chemical reaction kinetics and dynamics. The recent, promising work of Nakamura et al is the first stepping stone in time-resolved AP-HAXPES [374]. Further developments are necessary to achieve the improved time resolution necessary to follow fast reactions. In addition, the incorporation of grazing-incidence and standing-wave geometries into AP-HAXPES experiments has great potential to boost the interface signal in synchrotron AP-HAXPES experiments. Similarly, we expect the extension of ambient pressure environments into other HAXPES techniques. Such capabilities have recently been incorporated in soft X-ray photoemission electron microscopy (PEEM), for example, where working pressures of around 1 mbar can be achieved [378]. Combining AP-HAXPES and HAXPEEM could open up new routes to operando investigations of laterally inhomogeneous, industrially relevant catalytic and electrochemical systems with sub-micron spatial resolution.

\subsubsection{Operando HAXPES of electric and electrochemical} devices. Ongoing efforts, in particular at P22 at PETRA3, GALAXIES at SOLEIL, and BL36XU at SPring-8 [50, 57], are enabling us to not only study samples under static conditions, e.g. post-cycling of a battery, but to perform operando experiments under dynamic conditions. This enables insights into the behavior of a wide range of materials during operation in electric and electrochemical devices, including transistors and memristors, batteries, photovoltaics, and fuel cells.

HAXPES enables the study of the active layers of such devices, as well as crucial buried interfaces, through the higher probing depth resulting from the use of hard x-rays. In many device structures active layers are buried underneath electrode top layers impenetrable for photoelectrons with kinetic energies resulting from excitation with soft $x$-rays. The ability to apply an electrical bias to a device structure while it is in a photoemission experimental position enables the study of ionic and electronic processes as a function of said bias. The experiments discussed in the next paragraphs enable the direct correlation of changes to oxidation states, chemistry, elemental distribution, and electronic structure with electrical and device characteristics.

Resistive random-access memory (RRAM or ReRAM) based on memristors has been the subject of a number of operando HAXPES studies to date. The ability to probe the same sample under different biasing conditions is of particular interest for RRAM devices as they often show varied behavior ac [379]. In this particular system a redistribution of oxygen within the system is observed through the detailed investigation of the Ti $2 p$ core level and subtle changes in oxidation state distributions. By the nature of the excellent oxygen getter abilities of titanium, it oxidises, pulling oxygen away from the active $\mathrm{HfO}_{2}$ layer resulting in a local deficiency. Schroeder et al describe this behavior as a push-pull model of oxygen vacancies coupled with the formed $\mathrm{TiO}_{2}$ interfacial layer acting as an oxygen reservoir. Similar oxygen getter behavior was observed by Meunier et al in TiN/LaMnO $3+\delta(\mathrm{LMO}) / \mathrm{Pt}$ devices [380]. By following changes in the Ti and $\mathrm{O} 1 s$ core level spectra they were able to identify the oxygen exchange process taking place at the TiN/LMO interface. This study showcases the advantage of being able to access deeper core levels, in this case Ti $1 s$, to enable simpler data analysis and interpretation compared to the shallower, more commonly collected Ti $2 p$ spectra, which are complicated by the overlap of the $2 p_{3 / 2}$ and $1 / 2$ components of the core level.

Oxygen dynamics observed with operando HAXPES are not solely limited to metals or nitrides scavenging oxygen from metal oxide layers, but can also be followed across oxide-oxide interfaces. In the case of so-called tunnel RRAM devices, which are based on an insulating oxide layer deposited between a noble metal top electrode and a conductive metal oxide, it was long speculated that the switching observed resulted from an exchange of oxygen between the two oxide layers. This redistribution of oxygen atoms was thought to give rise to an ionic space charge, which in turn would modulate the barrier height and therefore the tunneling current. The operando HAXPES study by Arndt et al on a tunnel RRAM device based on yttria-stabilised zirconia (YSZ) as the insulating oxide layer and $\operatorname{Pr}_{1-x} \mathrm{Ca}_{x} \mathrm{MnO}_{3}$ (PCMO) as the conductive oxide layer finally provided conclusive evidence that this process does indeed take place and can explain the underlying mechanism of resistive switching in these devices [381]. By designing a special device array suited to operando studies and collecting core level spectra of the different device layers they were able to follow changes upon biasing and directly correlate them with electrical measurements.

Another related area where operando HAXPES has found application are interfaces between ferroelectric oxides and metals. Ferroelectric materials, such as $\mathrm{Pb}(M, \mathrm{Ti}) \mathrm{O}_{3}$, where $M$ can e.g. be $\mathrm{Zr}, \mathrm{Mg}$, or $\mathrm{Nb}$, exhibit spontaneous electric polarisation that is reversible by an external field, and can therefore be used in a wide range of electric devices. The physics of such device structures is determined by the metal-ferroelectric interface, and therefore it is crucial to understand the interactions and changes at these buried interfaces. By following the evolution of core level spectra in such devices during biasing, dynamic changes to the electronic structure of the interfaces can be identified and correlated to electrical behavior [382, 383].

It is worth noting, that operando HAXPES experiments are not solely limited to inorganic-based devices, but have also been successfully applied to the study of organic light emitting diodes (OLEDs), for example in the work by Ikeuchi et al who were able to probe the buried interface between a Ba cathode and a light-emitting polymer layer in an operating OLED 
device, shedding light on the dynamic behavior of charges and resulting differences in electronic structure [384].

Beyond electronic devices as discussed up to now, efforts are made to extend operando HAXPES to energy applications such as batteries and fuel cells. In a very recent work by Kiuchi and co-workers, they developed a dedicated manipulator and sample holder to allow electrochemical measurements of a battery inside the UHV chamber of the HAXPES endstation at beamline BL28XU at SPring-8 [385]. Al-deposited $\mathrm{LiCoO}_{2}$ thin film cells were investigated during charge-discharge profiles. Reversible changes observed in both core level and valence band spectra point toward $\mathrm{Co}$ and $\mathrm{O}$ anions contributing to the redox mechanisms taking place during battery cycling.

Beamline BL36XU at SPring-8 and the team of Toshihiko Yokoyama have been trailblazers in not only developing operando setups for fuel cells but also in combining them with ambient pressure environments [371, 373, 374]. They have explored both the interface between the Pt and the cathode catalysts as well as the role of sulfur in the poisoning of electrodes in polymer electrolyte fuel cells (PEFC). In their latest study they were able to show Pt oxidation state changes in a $\mathrm{Pt} / \mathrm{C}$ cathode catalyst upon an abrupt step of the cathode-anode voltage with $\mathrm{ms}$ time resolution. They collected time-resolved operando Pt $3 d_{3 / 2}$ and S $1 s$ core level spectra and could follow chemical changes over time both qualitatively and quantitatively. Again, the use of the deeper S $1 s$ core level showed clear advantages over the shallower, more routinely measured S $2 p$ core level.

The previously described approaches rely on the high probing depth of operando HAXPES to study functional layers e.g. under top electrodes. Recent efforts to include XSWs in total reflection geometry allow to gain a unique depth resolved view of layered device samples [386]. In this study ferroelectric memory devices based on $\mathrm{HfO}_{2}$ were investigated, which are one of the most promising candidates for future non-volatile memory devices. Previously, operando HAXPES had revealed changes in the conduction band offsets depending on the switching state of the ferroelectric layer [387]. By using XSWs and analysing core level shifts a detailed non-linear potential profile could be extracted for the two polarisation states. This allowed to localize different defect types at the oxide interfaces of the TiN/ $/ \mathrm{Hf}_{0.5} \mathrm{Zr}_{0.5} \mathrm{O}_{2} / \mathrm{W}$ device stack.

Almost all of the studies discussed here use excitation energies between 6 and $8 \mathrm{keV}$ and solely explore core level spectra. To date, only very few studies include the collection of valence band spectra during under operando conditions due to the low signal intensities from valence states. However, going forward, studies of the electronic structure close to the Fermi energy and across the valence band will surely provide crucial insights into electronic materials employed in devices. The prospect of combining operando setups with the timeresolved HAXPES in the nano- and picosecond regime, as discussed in sections 2.3 and 3.1.8, would provide direct dynamical measurements of charge carrier dynamics and even ultrafast electronic processes during device operation. This would provide unprecedented insights into the fundamental processes and their dynamic behavior, which form the foundation of modern device technologies.

\section{Outlook and future}

Without a pun intended, and based on the snapshot this review paper presents, we see a bright future for HAXPES and its variations. Technological developments of both X-ray sources and spectrometers are continuously expanding available measurement modalities and open up new opportunities for the application of the technique to an almost unlimited range of materials and related scientific questions. Based on ongoing developments and the expected fundamental advances they will enable the next decade will see a dramatic increase of performance of photoelectron spectroscopy in general and HAXPES in particular.

The new laboratory-based HAXPES systems will continue to increase in numbers and enable to broaden the user base and application of the technique across varying scientific areas. Continuing developments to increase the photon flux provided by laboratory sources will enable the transition from synchrotron to laboratory for the more photon-hungry variants of HAXPES, including ambient pressure HAXPES.

The advent of 4th generation synchrotron sources operating in the diffraction-limited domain, including existing and planned developments at MAX IV, ESRF-EBS, PETRA IV, Diamond-II, and SPring-8-II, will push synchrotron radiation to new physical limits. This new development will result in a further increase in photon intensities and provide significantly reduced beam sizes that will easily reach the nanometer scale. At the same time full polarisation control and crystal optical concepts providing higher energy resolutions will become more common. This opens the door to the investigation of laterally inhomogeneous samples on the nanoscale. With this, operando HAXPES of individual electronic devices of industrially relevant dimensions or MCDAD of single magnetic domains will become possible for the first time. Thanks to shorter acquisition times, HAXPES will continue to explore the temporal and spatial domains. Particularly in combination with nano-HAXPES, microscopic investigation of structured or inhomogeneous samples become very attractive. First steps in this direction are expected based on the development of achromatic capillary optics for multi keV photons providing sub-micron beam sizes [388]. Higher control over x-ray beam characteristics will also enable new analytical opportunities for example through the use of vortex beams discussed in section 3.2.2, which has not been used for XPS or HAXPES experiments, but promises new measurement opportunities for magnetic materials and the observation of new forms of dichroism.

Furthermore, diffraction-limited storage rings will enable AP-HAXPES studies in the crucial pressure regime of 1-10 bar. Using their small focus size below $1 \mu \mathrm{m}$ the size of a virtual cell can be matched to enable measurements at such pressures. This pressure range is crucial for the ongoing transformation of the chemical industry and energy production toward sustainable schemes based on water photocatalysis and 
electrolysis, where inherently lower input pressures are driving current developments in catalysts, which can operate under such conditions rather than the high pressure environments standard in classic catalytic applications. AP-HAXPES is set to evolve to handle higher pressures enable the operando study of catalysis under conditions exactly representing the needs of future applications.

Developments in free electron lasers enable key emerging applications of HAXPES, which concern dynamic, operando studies of materials, including for example the exploration of electronic devices and the related real time observation of ultra-fast processes on pico-, femto- and even attosecond time scales. X-ray pulses from FELs give access to fs and sub-fs core-level dynamics and allow 'locking-in' to the coupled coherent lattice motion, exploiting photoelectron diffraction and x-ray standing waves, as well as to the coupled spin dynamics using direct spin detection and MCDAD contrast [129, 136, 287].

Proof-of-principle experiments have been performed for a number of exciting emerging applications of time-resolved momentum microscopy, including ultrafast molecular orbital imaging and tracking transient changes of topological properties and orbital texture of out-of-equilibrium states of matter, where currently XUV excitation energies are used, but which could be translated into the hard X-ray regime in the future [198, 389-391]. A new powerful tool, that is already using hard x-rays, is emerging in the form of full-field photoelectron diffraction for structural analysis, which has been demonstrated in first static experiments using hard x-rays [203, 204]. A serious obstacle for time resolved studies is the Coulomb interaction of electrons confined in a small spatio-temporal phase-space volume. All attempts to improve the performance of experiments by pulse compression to gain higher time resolution, minimising the size of the photon spot in order to reach better spatial resolution, or increasing intensity for better signal-to-noise ratio, increase the Coulomb repulsion in the charge cloud. This dilemma is commonly referred to as the 'vacuum space-charge problem'. Space-charge interactions manifest themselves in shifts of kinetic energies and peak broadening, downgrading energy and angular/momentum resolution. In particular, pump-probe experiments suffer from the space-charge effects as pump-induced slow electrons are launched at a different instant and thus their forces on the photoelectrons depend on the pump-probe delay. Strategies of a posteriori correction or a priori suppression of the space charge shifts in time resolved hard x-ray PES are presently explored [202].

In parallel to the leaps made in source development, advances in photoelectron analyser and detection schemes are crucial. One area were fundamental advances are maybe not expected to be dramatic are the hemispherical analysers widely used. After almost a century of development, they have reached their principal limits with excellent performance, including sub-meV energy resolution [392, 393], and high angular resolution (translating into a $k$-resolution of $0.003 \AA^{-1}$ ) at rather small angular acceptance [394]. However, challenges remain to establish standardised procedures for kinetic energy scale calibration to enable closer comparison between different instruments. Ongoing efforts to develop quantification strategies for depth-dependent composition and distribution of chemical states, as well as strategies to extract information from larger depths through analysis of the inelastic background, are essential to the broader application of HAXPES.

The emergence of ToF momentum microscopy approaches is exciting and although the method is still in its infancy, the first experiments in the x-ray regime, show that it can already compete with traditional hemispherical setups, for example reaching a total energy resolution of $62 \mathrm{meV}$ at a photon energy of $\approx 6 \mathrm{keV}$ [196]. Thanks to its 3D recording architecture, the technique can deliver high data throughput, however, it places requirements on the time structure of the x-ray source. The time resolution of the present photoelectron detectors is in the range $<100 \mathrm{ps}$, and therefore, to resolve the high number of energy slices within recorded data arrays desired, sufficiently large gaps between adjacent $\mathrm{x}$-ray pulses are required. With most synchrotrons operating at 500 or $100 \mathrm{MHz}$ and corresponding gaps of up to $10 \mathrm{~ns}$ and below only up to 100 energy slices can be recorded, which is not sufficient for truly high resolution ToF momentum microscopy. However, by implementing an additional dispersive element, $\mathrm{x}$-ray sources with high pulse rates may still be used and first results taken with a dispersive-plus-ToF hybrid type of momentum microscope have recently been published [395]. This can be achieved by combining hemispherical analysers with ToF analysers, with the latter sitting behind the exit slit of the hemisphere. This dispersive-plus-ToF hybrid can improve the recording efficiency (in resolution and/or transmission) by 1-2 orders of magnitude and the first prototype will be installed at beamline I09 at Diamond.

As is obvious from the previous section in this review, different HAXPES modi are already combined to obtain ever more complete data sets and therefore understanding of materials. Clever engineering and optimised technological designs enable more and more the integration and combination of different HAXPES modi. Several opportunities also exist to combine HAXPES with other related techniques, such as diffraction or (coherent) scattering [71]. Ultimately, the field is moving toward a total HAXPES approach providing full energy, spin, angular, spatial, and temporal resolution exploiting full control over the incoming hard $\mathrm{x}$-ray photon beam. We predict the coming decade to be one of fast and significant advances in HAXPES, and are excited to see what a snapshot of the technique will look like in 2030.

\section{Acknowledgments}

CK acknowledges support from the Department of Chemistry, UCL. NKF acknowledges support from the Engineering and Physical Sciences Research Council (EP/L015277/1). AR acknowledges the support from the Analytical Chemistry Trust Fund for her CAMS-UK Fellowship. AXG acknowledges support from the U.S. Department of Energy, Office of Science, Office of Basic Energy Sciences, Materials Sciences and Engineering Division under Award Number DE-SC0019297. 
Part of this work was performed in the framework of the competence center FunMat-II that is financially supported by Vinnova (Grant No. 2016-05156). The Empa authors acknowledge financial support from the Swiss National Science Foundation (R'Equip program, Proposal No. 206021_182987). FOLJ, AL and HR acknowledge funding from The Swedish Energy Agency and The Swedish Research Council. AL acknowledges the support from the Swedish Research Council (Grants No. 2014-6463 and No. 2018-05336) and Marie Sklodowska Curie Actions (Cofund, Project INCA 600398). GS and KM acknowledge support from BMBF (projects 05K19UM1 and 05K19UM2) and DFG (German Research Foundation, Grant No. TRR 173 268565370, Project A02). KR acknowledges support from BMBF (Project No. 05K19FK1).

The authors would like to thank all beamline scientists who have provided up to date information on beamlines worldwide, including Tien-Lin Lee (DLS), Andrei Hloskovskii (PETRA III), Kenji Tamasaku, Shigenori Ueda, Hisao Kiuchi, Akira Yasui, Yasumasa Takagi, Tomoya Uruga, Satoru Suzuki, Masaki Oura, Masahiko Yoshiki, Masaaki Kobata, Takamasa Nonaka and Satoshi Yasuno (all SPring-8), Juan Rubio Zuazo (ESRF), Conan Weiland (NIST beamline at NSLS-II), JeanPascal Rueff (SOLEIL), Yongfeng Hu (CLS), Zhan Zhang and Denis Keane (APS), Roberto Félix and Mihaela Gorgoi (BESSY II), Thomas Huthwelker and Zbynek Novotny (SLS), Jaspreet Singh (Indus-2), and Yi Yu (SSRF). Thanks also goes to everyone who has supplied information on laboratorybased systems, including Tappei Nishihara (Meiji University), Ben Spencer and Wendy Flavell (University of Manchester), Louis Piper (Universities of Binghamton and Warwick), Hisao Makino (Kochi University) and Masashi Yoshimura (Hyogo Science and Technology Association Synchrotron Radiation Center). The authors would also like to thank Scienta Omicron, SPECS, PHI, and Kratos for providing information on commercial HAXPES systems. Furthermore, we thank Wolfgang Drube for fruitful discussions and feedback during the writing stages of this manuscript. We are also grateful to Hans Siegbahn and Svante Svensson for discussions on the history of PES.

\section{Data availability statement}

The data that support the findings of this study are available upon reasonable request from the authors.

\section{ORCID iDs}

Curran Kalha (D) https://orcid.org/0000-0002-3387-6880

Nathalie K Fernando (D) https://orcid.org/0000-0001-78141741

Prajna Bhatt (D) https://orcid.org/0000-0002-5699-2841

Fredrik O L Johansson (D) https://orcid.org/0000-0002-64711093

Andreas Lindblad (1D https://orcid.org/0000-0002-9188-9604 León Zendejas Medina (D) https://orcid.org/0000-0002-92045999
Rebecka Lindblad (D) https://orcid.org/0000-0001-6162-1167 Sebastian Siol (1) https://orcid.org/0000-0002-0907-6525

Lars P H Jeurgens (D) https://orcid.org/0000-0002-0264-9220 Claudia Cancellieri (D) https://orcid.org/0000-0003-41244362

Kai Rossnagel (1) https://orcid.org/0000-0001-5107-0090

Katerina Medjanik (D) https://orcid.org/0000-0001-9646-4157 Gerd Schönhense (D) https://orcid.org/0000-0002-8921-2901 Marc Simon (D) https://orcid.org/0000-0002-2525-5435

Alexander X Gray (D) https://orcid.org/0000-0002-7634-4294

Slavomír Nemšák (D) https://orcid.org/0000-0002-6103-2925

Patrick Lömker (D) https://orcid.org/0000-0002-5297-710X

Christoph Schlueter (D) https://orcid.org/0000-0002-87453074

Anna Regoutz (D) https://orcid.org/0000-0002-3747-3763

\section{References}

[1] Hertz H 1887 Ann. Phys. Chem. 267 983-1000

[2] Hallwachs W 1888 Ann. Phys. Chem. 269 301-12

[3] Lenard P 1902 Ann. Phys. 313 149-98

[4] Einstein A 1905 Ann. Phys. 322 132-48

[5] Rutherford E 1914 London, Edinburgh Dublin Phil. Mag. J. Sci. 28 305-19

[6] Siegbahn K and Svartholm N 1946 Nature 157 872-3

[7] Siegbahn K and Edvarson K 1956 Nucl. Phys. 1 137-59

[8] Nordling C, Sokolowski E and Siegbahn K 1957 Phys. Rev. $1051676-7$

[9] Sokolowski E, Nordling C and Siegbahn K 1958 Phys. Rev. 110776

[10] Nordling C, Sokolowski E and Siegbahn K 1958 Arkiv Fysik 13483

[11] Hagström S, Nordling C and Siegbahn K 1964 Z. Phys. 178 439-44

[12] Nordling C, Hagström S and Siegbahn K 1964 Z. Phys. 178 433-8

[13] Siegbahn K et al 1967 ESCA: Atomic, Molecular, and Solid State Structure Studied by Means of Electron Spectroscopy (Uppsala: Almqvist and Wiksells)

[14] Fadley C S, Hagström S B M, Hollander J M, Klein M P and Shirley D A 1967 Science 157 1571-3

[15] Panaccione G et al 2005 Nucl. Instrum. Methods Phys. Res. A $54756-63$

[16] Dallera C, Braicovich L, Duò L, Palenzona A, Panaccione G, Paolicelli G, Cowie B C C and Zegenhagen J 2005 Nucl. Instrum. Methods Phys. Res. A 547 113-23

[17] Hagström S, Nordling C and Siegbahn K 1964 Phys. Lett. 9 235-6

[18] Lindau I, Pianetta P, Doniach S and Spicer W E 1974 Nature $250214-5$

[19] Lindau I, Pianetta P, Yu K and Spicer W E 1975 Phys. Lett. A 54 47-8

[20] Drube W, Lessmann A and Materlik G 1992 Rev. Sci. Instrum. $631138-41$

[21] Drube W, Treusch R and Materlik G 1995 Phys. Rev. Lett. 74 $42-5$

[22] Brown G S, Chen M H, Crasemann B and Ice G E 1980 Phys. Rev. Lett. 45 1937-40

[23] Cox L E, Ellis W P, Cowan R D, Allen J W and Oh S-J 1985 Phys. Rev. B 31 2467-71

[24] Armen G B, Åberg T, Levin J C, Crasemann B, Chen M H, Ice G E and Brown G S 1985 Phys. Rev. Lett. 54 1142-5

[25] Sekiyama A, Iwasaki T, Matsuda K, Saitoh Y, Ônuki Y and Suga S 2000 Nature 403 396-8 
[26] Yabashi M et al 2001 Nucl. Instrum. Methods Phys. Res. A 467-468 678-81

[27] Tamasaku K, Tanaka Y, Yabashi M, Yamazaki H, Kawamura N, Suzuki M and Ishikawa T 2001 Nucl. Instrum. Methods Phys. Res. A 467-468 686-9

[28] Cai Y Q, Chow P, Chen C C, Ishii H, Tsang K L, Kao C C, Liang K S and Chen C T 2004 AIP Conf. Proc. 705 340-3

[29] Kobayashi K et al 2003 Appl. Phys. Lett. 83 1005-7

[30] Takata Y et al 2004 Appl. Phys. Lett. 84 4310-2

[31] Kobayashi K 2005 Nucl. Instrum. Methods Phys. Res. A 547 98-112

[32] Thiess S, Kunz C, Cowie B C C, Lee T-L, Renier M and Zegenhagen J 2004 Solid State Commun. 132 589-94

[33] Torelli P et al 2005 Rev. Sci. Instrum. 76023909

[34] Sacchi M et al 2005 Phys. Rev. B 71155117

[35] Panaccione G et al 2006 Phys. Rev. Lett. 97116401

[36] Ablett J M et al 2013 J. Phys.: Conf. Ser. 425052007

[37] Schlueter C, Orgiani P, Lee T L, Petrov A Y, Galdi A, Davidson B A, Zegenhagen J and Aruta C 2012 Phys. Rev. B 86155102

[38] Boujamaa R et al 2012 J. Appl. Phys. 111054110

[39] Lee T L, Warren S, Cowie B C and Zegenhagen J 2006 Phys. Rev. Lett. 96046103

[40] Zegenhagen J et al 2010 J. Electron Spectrosc. Relat. Phenom. 178-179 258-67

[41] Fadley C S 2005 Nucl. Instrum. Methods Phys. Res. A 547 24-41

[42] Kobayashi K 2009 Nucl. Instrum. Methods Phys. Res. A 601 $32-47$

[43] Kövér L 2010 J. Electron Spectrosc. Relat. Phenom. 178-179 241-57

[44] Panaccione G and Kobayashi K 2012 Surf. Sci. 606 125-9

[45] Drube W 2013 J. Electron Spectrosc. Relat. Phenom. 190 125-6

[46] Fadley C S 2013 J. Electron Spectrosc. Relat. Phenom. 190 165-79

[47] Weiland C, Rumaiz A K, Pianetta P and Woicik J C 2016 J. Vac. Sci. Technol. A 34030801

[48] Woicik J C 2016 Hard X-Ray Photoelectron Spectroscopy (HAXPES) 1st edn vol 59 (Berlin: Springer)

[49] Rubio-Zuazo J R and Castro G R 2005 Nucl. Instrum. Methods Phys. Res. A 547 64-72

[50] Rueff J-P, Rault J E, Ablett J M, Utsumi Y and Céolin D 2018 Synchrotron Radiat. News 31 4-9

[51] Céolin D et al 2013 J. Electron Spectrosc. Relat. Phenom. 190 188-92

[52] Rueff J-P et al 2015 J. Synchrotron Radiat. 22 175-9

[53] Hendel S, Schäfers F, Hävecker M, Reichardt G, Scheer M, Bahrdt J and Lips K 2016 The EMIL project at BESSY II: beamline design and performance AIP Conf. Proc. vol 1741 (American Institute of Physics Inc.)

[54] Schaefers F, Mertin M and Gorgoi M 2007 Rev. Sci. Instrum. 78123102

[55] Gorgoi M et al 2009 Nucl. Instrum. Methods Phys. Res. A 601 $48-53$

[56] Schlueter C et al 2018 Synchrotron Radiat. News 31 29-35

[57] Schlueter C et al 2019 AIP Conf. Proc. 2054040010

[58] Novotny Z et al 2020 Rev. Sci. Instrum. 91023103

[59] Lee T-L and Duncan D A 2018 Synchrotron Radiat. News 31 $16-22$

[60] Ikenaga E, Yasui A, Kawamura N, Mizumaki M, Tsutsui S and Mimura K 2018 Synchrotron Radiat. News 31 10-5

[61] Tsuei K D et al 2010 SPring-8 Information 15 252-9

[62] Ueda S, Katsuya Y, Tanaka M, Yoshikawa H, Yamashita Y, Ishimaru S, Matsushita Y and Kobayashi K 2010 Present status of the NIMS contract beamline BL15XU at SPring-8 AIP Conf. Proc. 1234 403-6

[63] Shobu T, Tozawa K, Shiwaku H, Konishi H, Inami T, Harami T and Mizuki J 2007 Wide band energy beamline using Si
(111) crystal monochromators at BL22XU in SPring-8 AIP Conf. Proc. 879 902-6

[64] Tanida H et al 2014 J. Synchrotron Radiat. 21 268-72

[65] Takata Y et al 2005 Nucl. Instrum. Methods Phys. Res. A 547 $50-5$

[66] Uruga T, Tada M, Sekizawa O, Takagi Y, Yokoyama T and Iwasawa Y 2019 Chem. Rec. 19 1444-56

[67] Yasuno S, Oji H, Koganezawa T and Watanabe T 2016 Hard $\mathrm{X}$-ray photoelectron spectroscopy equipment developed at beamline BL46XU of SPring-8 for industrial researches AIP Conf. Proc. vol 1741 (American Institute of Physics Inc.)) 030020

[68] Ikenaga E, Kobata M, Matsuda H, Sugiyama T, Daimon H and Kobayashi K 2013 J. Electron Spectrosc. Relat. Phenom. $190180-7$

[69] Jagannath G U K, Goutam U K, Sharma R K, Singh J, Dutta K, Sule U S, Pradeep P and Gadkari S C 2018 J. Synchrotron Radiat. 25 1541-7

[70] Xiao Q, Cui X, Shi Y, Hu Y, Sham T-K, Piao H and McMahon J 2015 Can. J. Chem. 93 113-7

[71] Eres G, Rouleau C M, Lu Q, Zhang Z, Benda E, Lee H N, Tischler J Z and Fong D D 2019 Rev. Sci. Instrum. 90 093902

[72] Weiland C et al 2018 Synchrotron Radiat. News 31 23-8

[73] Kobata M, Pr̂́s I, Iwai H, Yamazui H, Takahashi H, Suzuki M, Matsuda H, Daimon H and Kobayashi K 2010 Anal. Sci. 26 227-32

[74] Kobayashi K, Kobata M and Iwai H 2013 J. Electron Spectrosc. Relat. Phenom. 190 210-21

[75] Jackson A J, Ganose A M, Regoutz A, Egdell R G and Scanlon D O 2018 J. Open Source Softw. 3773

[76] Scofield J H 1973 Theoretical Photoionization Cross Sections from 1 to $1500 \mathrm{keV}$ Tech. Rep. U.S. Atomic Energy Commission, Divison of Technical Information Extension

[77] Yeh J J and Lindau I 1985 At. Data Nucl. Data Tables 32 1-155

[78] Trzhaskovskaya M B and Yarzhemsky V G 2018 At. Data Nucl. Data Tables 119 99-174

[79] Trzhaskovskaya M B and Yarzhemsky V G 2019 At. Data Nucl. Data Tables 129-130 101280

[80] Kalha C, Fernando N K and Regoutz A 2020 Digitisation of Scofield Hartree-Fock Normalizations and Binding Energies Tabulated Data

[81] Kalha C, Fernando N K and Regoutz A 2020 Digitisation of Scofield Photoionisation Cross Section Tabulated Data

[82] Kalha C, Fernando N K, Hernandez C V, Kurtulus E E, Li J, Zhou Y and Regoutz A 2020 Digitisation of Yeh and Lindau Photoionisation Cross Section Tabulated Data

[83] Willis J, Kalha C, Trzhaskovskaya M B, Yarzhemsky V G, Scanlon D and Regoutz A 2020 Digitisation of Trzhaskovskaya Dirac-Fock Photoionisation Parameters for HAXPES Applications

[84] Willis J, Kalha C, Trzhaskovskaya M B, Yarzhemsky V G, Scanlon D and Regoutz A 2020 Digitisation of Trzhaskovskaya Dirac-Fock Photoionisation Parameters for HAXPES Applications, Part II

[85] Lebens-Higgins Z W et al 2019 Sci. Rep. 917720

[86] Mulazzi M, Reichmann F, Becker A, Klesse W M, Alippi P, Fiorentini V, Parisini A, Bosi M and Fornari R 2019 APL Mater. 7022522

[87] Nishihara T, Kamioka T, Kanai H, Ohshita Y, Yasuno S, Hirosawa I and Ogura A 2019 ECS J. Solid State Sci. Technol. 8 Q101-5

[88] Nishihara T, Yokogawa R, Otsuki Y, Kagaya M and Ogura A 2020 ECS Trans. 98 113-20

[89] Berens J et al 2020 J. Phys. Energy 2035001

[90] Siol S, Mann J, Newman J, Miyayama T, Watanabe K, Schmutz P, Cancellieri C and Jeurgens L P H 2020 Surf. Interface Anal. 52 802-10 
[91] Sambalova O, Billeter E, Mann J, Miyayama T, Burnat D, Heel A, Bleiner D and Borgschulte A 2020 Surf. Interface Anal. 52 811-7

[92] Karis O, Svensson S, Rusz J, Oppeneer P M, Gorgoi M, Schäfers F, Braun W, Eberhardt W and Mårtensson N 2008 Phys. Rev. B 78233105

[93] Miedema P S, Borgatti F, Offi F, Panaccione G and De Groot F M F 2015 J. Electron Spectrosc. Relat. Phenom. 203 $8-13$

[94] Moslemzadeh N, Beamson G, Tsakiropoulos P, Watts J F, Haines S R and Weightman P 2006 J. Electron Spectrosc. Relat. Phenom. 152 129-33

[95] Vobornik I et al 2014 J. Phys. Chem. C 118 12333-9

[96] Ghiasi M, Hariki A, Winder M, Kuneš J, Regoutz A, Lee T L, Hu Y, Rueff J P and De Groot F M 2019 Phys. Rev. B 100 075146

[97] Woicik J C, Weiland C, Jaye C, Fischer D A, Rumaiz A K, Shirley E L, Kas J J and Rehr J J 2020 Phys. Rev. B 101 245119

[98] Fuggle J C and Mårtensson N 1980 J. Electron Spectrosc. Relat. Phenom. 21 275-81

[99] Cardona M, Ley L, Schaich W L, Manson S T, Shirley D A, Wertheim G K, Citrin P H and Smith N V 1978 Photoemission in Solids I vol 26 (Berlin: Springer)

[100] Regoutz A, Mascheck M, Wiell T, Eriksson S K, Liljenberg C, Tetzner K, Williamson B A D, Scanlon D O and Palmgren P 2018 Rev. Sci. Instrum. 89073105

[101] Wagner C D 1975 Faraday Discuss. Chem. Soc. 60291

[102] Wagner C D and Joshi A 1988 J. Electron Spectrosc. Relat. Phenom. 47 283-313

[103] Wagner C D, Passoja D E, Hillery H F, Kinisky T G, Six H A, Jansen W T and Taylor J A 1982 J. Vac. Sci. Technol. 21 933-44

[104] Snijders P C, Jeurgens L P H and Sloof W G 2005 Surf. Sci. $58998-105$

[105] Kövér L, Varga D, Cserny I, Tóth J and Tökési K 1992 Surf. Interface Anal. 19 9-15

[106] Razek S A, Popeil M R, Wangoh L, Rana J, Suwandaratne N, Andrews J L, Watson D F, Banerjee S and Piper L F J 2020 Electron. Struct. 2023001

[107] Kraut E A, Grant R W, Waldrop J R and Kowalczyk S P 1983 Phys. Rev. B 28 1965-77

[108] Werner W S M 1995 Surf. Interface Anal. 23 696-704

[109] Gunter P L J and Niemantsverdriet J W 1995 Appl. Surf. Sci. 89 69-76

[110] Hemberg O, Otendal M and Hertz H M 2003 Appl. Phys. Lett. 83 1483-5

[111] Otendal M, Tuohimaa T, Vogt U and Hertz H M 2008 Rev. Sci. Instrum. 79 16-9

[112] Marziani M, Gambaccini M, Di Domenico G, Taibi A and Cardarelli P 2014 Appl. Radiat. Isot. 92 32-6

[113] Spencer B et al 2021 Appl. Surf. Sci. 541148635

[114] Kim G, Yu Y, Lim H, Jeong B, Lee J, Baik J, Mun B S and Kim K-j. 2020 J. Synchrotron Radiat. 27 507-14

[115] Shard A G, Counsell J D P, Cant D J H, Smith E F, Navabpour $\mathrm{P}$, Zhang X and Blomfield C J 2019 Surf. Interface Anal. 51 $763-73$

[116] Blundell R K, Delorme A E, Smith E F and Licence P 2016 Phys. Chem. Chem. Phys. 18 6122-31

[117] Pleul D, Frenzel R, Eschner M and Simon F 2003 Anal. Bioanal. Chem. 375 1276-81

[118] Roberts A J and Moffitt C E 2019 J. Electron Spectrosc. Relat. Phenom. 231 68-74

[119] Isaacs M A et al 2020 Nat. Catal. 3 921-31

[120] Makino H and Shimizu H 2018 Appl. Surf. Sci. 439 839-44

[121] Craciun D, Vasile B S, Lambers E, Makino H and Craciun V 2020 Surf. Eng. 36 326-33

[122] Magari Y, Makino H, Hashimoto S and Furuta M 2020 Appl. Surf. Sci. 512144519
[123] Wang J, Shin Y, Paudel J R, Grassi J D, Sah R K, Yang W, Karapetrova E, Zaidan A, Strocov V N, Klewe C, Shafer P, Gray A X, Rondinelli J M and May S J et al 2021 StrainInduced Anion-Site Occupancy in Perovskite Oxyfluoride Films Chem. Mater. 33 1811-20

[124] Oloff L P, Oura M, Chainani A and Rossnagel K 2016 Femtosecond time-resolved HAXPES Hard X-Ray Photoelectron Spectroscopy (HAXPES) 1st edn vol 59 ed J C Woicik (Berlin: Springer) ch 20 pp 555-68

[125] Pressacco F et al 2018 Struct. Dyn. 5034501

[126] Ozawa K, Yamamoto S, D'angelo M, Natsui Y, Terashima N, Mase K and Matsuda I 2019 J. Phys. Chem. C 123 4388-95

[127] Borgwardt M et al 2020 J. Phys. Chem. Lett. 11 5476-81

[128] Pincelli T et al 2019 Phys. Rev. B 100045118

[129] Dendzik M et al 2020 Phys. Rev. Lett. 125096401

[130] Siefermann K R et al 2014 J. Phys. Chem. Lett. 5 2753-9

[131] Oloff L P et al 2016 Sci. Rep. 635087

[132] Silly M G et al 2017 J. Synchrotron Radiat. 24 886-97

[133] Ogawa M et al 2012 Rev. Sci. Instrum. 83023109

[134] Neppl S and Gessner O 2015 J. Electron Spectrosc. Relat. Phenom. 200 64-77

[135] Hellmann S et al 2010 Phys. Rev. Lett. 105187401

[136] Hellmann S et al 2012 New J. Phys. 14013062

[137] Kutnyakhov D et al 2020 Rev. Sci. Instrum. 91013109

[138] Oura M et al 2014 J. Synchrotron Radiat. 21 183-92

[139] Oloff L-P et al 2014 New J. Phys. 16123045

[140] Verna A, Greco G, Lollobrigida V, Offi F and Stefani G 2016 J. Electron Spectrosc. Relat. Phenom. 209 14-25

[141] Medjanik K et al 2017 Nat. Mater. 16 615-21

[142] Lollobrigida V, Greco G, Simeone D, Offi F, Verna A and Stefani G 2015 J. Electron Spectrosc. Relat. Phenom. 205 98-105

[143] Powell C J and Jablonski A 2009 Nucl. Instrum. Methods Phys. Res. A 601 54-65

[144] Powell C J 2020 J. Vac. Sci. Technol. A 38023209

[145] Seah M P and Dench W A 1979 Surf. Interface Anal. 12

[146] Tanuma S, Powell C J and Penn D R 1988 Surf. Interface Anal. 11 577-89

[147] Tanuma S, Powell C J and Penn D R 2003 Surf. Interface Anal. $35268-75$

[148] Tanuma S, Yoshikawa H, Shinotsuka H and Ueda R 2013 J. Electron Spectrosc. Relat. Phenom. 190 127-36

[149] Risterucci P, Renault O, Martinez E, Detlefs B, Zegenhagen J, Grenet G and Tougaard S 2014 Surf. Interface Anal. 46 906-10

[150] Jablonski A and Powell C J 2002 Surf. Sci. Rep. 47 33-91

[151] Jablonski A and Powell C J 2009 J. Vac. Sci. Technol. A 27 253-61

[152] Jablonski A and Powell C J 2015 J. Electron Spectrosc. Relat. Phenom. 199 27-37

[153] Jablonski A and Powell C J 2020 J. Phys. Chem. Ref. Data 49 033102

[154] Solokha V, Lee T-L, Wilson A, Hingerl K and Zegenhagen J 2018 J. Electron Spectrosc. Relat. Phenom. 225 28-35

[155] Rubio-Zuazo J and Castro G R 2008 Surf. Interface Anal. 40 $1438-43$

[156] Shinotsuka H, Da B, Tanuma S, Yoshikawa H, Powell C J and Penn D R 2017 Surf. Interface Anal. 49 238-52

[157] Ottosson N, Faubel M, Bradforth S E, Jungwirth P and Winter B 2010 J. Electron Spectrosc. Relat. Phenom. 177 60-70

[158] Powell C J and Jablonski A 2010 J. Electron Spectrosc. Relat. Phenom. 178-179 331-46

[159] Ouardi S et al 2009 J. Phys. D: Appl. Phys. 42084011

[160] Young B T, Heskett D R, Nguyen C C, Nie M, Woicik J C and Lucht B L 2015 ACS Appl. Mater. Interfaces 7 20004-11

[161] Payne D J et al 2009 J. Electron Spectrosc. Relat. Phenom. 169 26-34

[162] Mukherjee S et al 2016 Phys. Rev. B 93 26-30

[163] Pincelli T et al 2017 Nat. Commun. 816051 
[164] Drube W, Grehk T M, Thieß S, Pradhan G B, Varma H R, Deshmukh P C and Manson S T 2013 J. Phys. B: At. Mol. Opt. Phys. $\mathbf{4 6} 5006$

[165] Zendejas Medina L, Riekehr L and Jansson U 2020 Surf. Coat. Technol. 403126323

[166] Karis O, Nilsson A, Weinelt M, Wiell T, Puglia C, Wassdahl N, Mårtensson N, Samant M and Stöhr J 1996 Phys. Rev. Lett. 76 1380-3

[167] Brühwiler P A, Karis $\mathrm{O}$ and Mårtensson N 2002 Rev. Mod. Phys. 74 703-40

[168] Johansson F O, Sassa Y, Edvinsson T and Lindblad A 2020 arXiv:2003.13394v1 [cond-mat.mtrl-sci]

[169] Johansson F O L, Ivanović M, Svanström S, Cappel U B, Peisert H, Chassé T and Lindblad A 2018 J. Phys. Chem. C 122 12605-14

[170] Johansson F O L, Cappel U B, Fondell M, Han Y, Gorgoi M, Leifer K and Lindblad A 2020 Phys. Chem. Chem. Phys. 22 10335-42

[171] Johansson F O, Chen X, Eriksson O, Sanyal B and Lindblad A 2020 Phys. Rev. B 102035165

[172] Johansson F O L 2020 Core-hole clock spectroscopy using hard x-rays: exciting states in condensed matter PhD Thesis University of Uppsala

[173] Panaccione G et al 2005 J. Phys.: Condens. Matter 17 2671-9

[174] Johansson E M J, Odelius M, Gorgoi M, Karis O, Ovsyannikov R, Schäfers F, Svensson S, Siegbahn H and Rensmo H 2008 Chem. Phys. Lett. 464 192-7

[175] Johansson E M J, Odelius M, Plogmaker S, Gorgoi M, Svensson S, Siegbahn H and Rensmo H 2010 J. Phys. Chem. C 114 10314-22

[176] Mudd J J, Lee T L, Muñoz-Sanjosé V, Zúñiga-Pérez J, Payne D J, Egdell R G and McConville C F 2014 Phys. Rev. B 89165305

[177] Regoutz A et al 2019 Phys. Rev. Mater. 3025001

[178] Takata Y et al 2008 Phys. Rev. Lett. 101137601

[179] Suga S 2008 Appl. Phys. A 92 479-85

[180] Suga S et al 2009 New J. Phys. 11073025

[181] Taguchi M, Takata Y and Chainani A 2013 J. Electron Spectrosc. Relat. Phenom. 190 242-8

[182] Tanuma S, Powell C J and Penn D R 2011 Surf. Interface Anal. 43 689-713

[183] Shevchik N J 1977 Phys. Rev. B 16 3428-42

[184] Hussain Z, Fadley C S, Kono S and Wagner L F 1980 Phys. Rev. B 22 3750-66

[185] Plucinski L, Minár J, Sell B C, Braun J, Ebert H, Schneider C M and Fadley C S 2008 Phys. Rev. B 78035108

[186] Papp C, Plucinski L, Minar J, Braun J, Ebert H, Schneider C M and Fadley C S 2011 Phys. Rev. B 84045433

[187] Braun J, Minár J, Mankovsky S, Strocov V N, Brookes N B, Plucinski L, Schneider C M, Fadley C S and Ebert H 2013 Phys. Rev. B 88205409

[188] Braun J, Minár J and Ebert H 2018 Phys. Rep. 740 1-34

[189] White R C, Fadley C S, Sagurton M and Hussain Z 1986 Phys. Rev. B 34 5226-38

[190] Herman G S, Tran T T, Higashiyama K and Fadley C S 1992 Phys. Rev. Lett. 68 1204-7

[191] Gray A X et al 2011 Nat. Mater. 10 759-64

[192] Xu S-Y et al 2015 Science 349 613-7

[193] Gray A X et al 2012 Nat. Mater. 11 957-62

[194] Nemšák S et al 2018 Nat. Commun. 93306

[195] Rattanachata A et al 2020 arXiv:2012.02863v1 [physics.app-ph]

[196] Medjanik K et al 2019 J. Synchrotron Radiat. 26 1996-2012

[197] Fedchenko O et al 2019 New J. Phys. 21013017

[198] Scholz M et al 2019 arXiv:1907.10434v1 [cond-mat.mes-hall]

[199] Vasilyev D, Medjanik K, Babenkov S, Ellguth M, Schönhense G and Elmers H-J 2020 J. Phys.: Condens. Matter 32 135501

[200] Babenkov S et al 2019 Commun. Phys. 2107
[201] Schönhense G, Medjanik K, Babenkov S, Vasilyev D, Ellguth M, Fedchenko O, Chernov S, Schönhense B and Elmers H J 2020 Commun. Phys. 345

[202] Schönhense B et al 2018 New J. Phys. 20033004

[203] Fedchenko O et al 2019 New J. Phys. 21113031

[204] Fedchenko O et al 2020 New J. Phys. 22103002

[205] Medjanik K et al 2021 Phys. Rev. B 103075107

[206] Šmejkal L, Železný J, Sinova J and Jungwirth T 2017 Phys. Rev. Lett. 118106402

[207] Elmers H J et al 2020 ACS Nano 14 17554-64

[208] Hayami S, Yatsushiro M, Yanagi Y and Kusunose H 2018 Phys. Rev. B 98165110

[209] Siegbahn K, Gelius U, Siegbahn H and Olson E 1970 Phys. Scr. $1272-6$

[210] Fadley C S and Bergström S A L 1971 Phys. Lett. A 35 $375-6$

[211] Fadley C S 1975 Faraday Discuss. Chem. Soc. 60 18-29

[212] Fadley C S 1987 Phys. Scr. T 17 39-49

[213] Fadley C S, Van Hove M A, Hussain Z and Kaduwela A P 1995 J. Electron Spectrosc. Relat. Phenom. 75 273-97

[214] Osterwalder J 2013 Electron based methods: 3.2.2 photoelectron spectroscopy and diffraction Surface and Interface Science vol 1 ed K Wandelt (New York: Wiley) ch 3.2 pp 151-214

[215] Woodruff D P 2010 J. Electron Spectrosc. Relat. Phenom. 178-179 186-94

[216] Fadley C S 2010 J. Electron Spectrosc. Relat. Phenom. 178-179 2-32

[217] Westphal C 2003 Surf. Sci. Rep. 50 1-106

[218] Fadley C S and Nemšák S 2014 J. Electron Spectrosc. Relat. Phenom. 195 409-22

[219] Eastman D E, Donelon J J, Hien N C and Himpsel F J 1980 Nucl. Instrum. Methods 172 327-36

[220] Daimon H 1990 Rev. Sci. Instrum. 61205

[221] Matsuda H et al 2014 J. Electron Spectrosc. Relat. Phenom. $195382-98$

[222] Daimon H, Nakatani T, Imada S, Suga S, Kagoshima Y and Miyahara T 1993 Japan. J. Appl. Phys. 32 1480-3

[223] Matsui F, Matsushita T and Daimon H 2014 J. Electron Spectrosc. Relat. Phenom. 195 347-60

[224] Matsui F, Nishikawa H, Daimon H, Muntwiler M, Takizawa M, Namba H and Greber T 2018 Phys. Rev. B 97 045430

[225] Hoesch M et al 2020 in preparation

[226] Trehan R, Osterwalder J and Fadley C S 1987 J. Electron Spectrosc. Relat. Phenom. 42 187-215

[227] García de Abajo F J, Van Hove M A and Fadley C S 2001 Phys. Rev. B 63075404

[228] Woodruff D P and Bradshaw A M 1994 Rep. Prog. Phys. 57 1029-80

[229] Winkelmann A, Fadley C S and Garcia de Abajo F J 2008 New J. Phys. 10113002

[230] Pendry J B 1976 Surf. Sci. 57 679-705

[231] Pendry J and Hopkinson J 1978 J. Phys. Colloq. 39 142-8

[232] Hopkinson J F L, Pendry J B and Titterington D J 1980 Comput. Phys. Commun. 19 69-92

[233] Osterwalder J, Fasel R, Stuck A, Aebi P and Schlapbach L 1994 J. Electron Spectrosc. Relat. Phenom. 68 1-18

[234] Matsushita T, Muro T, Yokoya T, Terashima K, Kato Y, Matsui H, Maejima N, Hashimoto Y and Matsui F 2020 Phys. Status SolidiB 2572000117

[235] Sinkovic B, Hermsmeier B and Fadley C S 1985 Phys. Rev. Lett. 55 1227-30

[236] Hermsmeier B, Osterwalder J, Friedman D J and Fadley C S 1989 Phys. Rev. Lett. 62 478-81

[237] Curcio D et al 2020 in preparation

[238] Sato S, Kataoka K, Jinnouchi R, Takahashi N, Sekizawa K, Kitazumi K, Ikenaga E, Asahi R and Morikawa T 2018 Phys. Chem. Chem. Phys. $2011342-6$ 
[239] Shindo S, Shuang Y, Hatayama S, Saito Y, Fons P, Kolobov A V, Kobayashi K and Sutou Y 2020 J. Appl. Phys. 128 165105

[240] Oshime N et al 2020 Sci. Rep. 1010702

[241] Sing M et al 2009 Phys. Rev. Lett. 102176805

[242] Ozawa K, Kakubo T, Amino N, Mase K, Ikenaga E and Nakamura T 2017 Langmuir 33 9582-9

[243] ViolBarbosa C E, Ouardi S, Fecher G H, Ebke D and Felser C 2013 J. Electron Spectrosc. Relat. Phenom. 189 146-51

[244] Bauer E 2001 J. Electron Spectrosc. Relat. Phenom. 114-116 975-87

[245] Bauer E 2001 J. Phys.: Condens. Matter 13 11391-404

[246] Schönhense G 1999 J. Phys.: Condens. Matter 11 9517-47

[247] Patt M 2016 Bulk and surface sensitive energy-filtered photoemission microscopy using synchrotron radiation for the study of resistive switching memories PhD Thesis Universität Duisburg-Essen

[248] Wiemann C, Patt M, Cramm S, Escher M, Merkel M, Gloskovskii A, Thiess S, Drube W and Schneider C M 2012 Appl. Phys. Lett. 100223106

[249] Kindsmüller A, Schmitz C, Wiemann C, Skaja K, Wouters D J, Waser R, Schneider C M and Dittmann R 2018 APL Mater. 6046106

[250] Bauer E 1992 Appl. Surf. Sci. 60-61 350-8

[251] Bauer E 1994 Rep. Prog. Phys. 57 895-938

[252] Tusche C, Chen Y-J, Schneider C M and Kirschner J 2019 Ultramicroscopy 206112815

[253] Tusche C, Chen Y-J, Plucinski L and Schneider C M 2020 e-J. Surf. Sci. Nanotechnol. 18 48-56

[254] Gloskovskii A et al 2012 J. Electron Spectrosc. Relat. Phenom. $18547-52$

[255] Patt M, Wiemann C, Weber N, Escher M, Gloskovskii A, Drube W, Merkel M and Schneider C M 2014 Rev. Sci. Instrum. 85113704

[256] Escher M et al 2005 J. Electron Spectrosc. Relat. Phenom. 144-147 1179-82

[257] Tougaard S 1998 Surf. Interface Anal. 26 249-69

[258] Tougaard S 2010 Energy loss in XPS: fundamental processes and applications for quantification, non-destructive depth profiling and 3D imaging J. Electron Spectrosc. Relat. Phenom. 178128

[259] Risterucci P, Renault O, Martinez E, Detlefs B, Delaye V, Zegenhagen J, Gaumer C, Grenet G and Tougaard S 2014 Appl. Phys. Lett. 104051608

[260] Renault O, Zborowski C, Risterucci P, Wiemann C, Grenet G, Schneider C M and Tougaard S 2016 Appl. Phys. Lett. 109 011602

[261] Långberg M et al 2019 J. Electrochem. Soc. 166 C3336-40

[262] Kessler J 1976 Polarized Electrons (Berlin: Springer)

[263] Kirschner J 1985 Springer Tracts in Modern Physics: Polarized Electrons at Surfaces vol 106 (Berlin: Springer)

[264] Feder R 1985 Polarized Electrons in Surface Physics (Singapore: World Scientific)

[265] Heinzmann U and Dil J H 2012 J. Phys.: Condens. Matter 24 173001

[266] Seddon E A 2014 Spin-resolved valence photoemission Handbook of Spintronics ed Y Xu, D D Awschalom and Y Nitta (Berlin: Springer) pp 1-75

[267] Bänninger U, Busch G, Campagna M and Siegmann H C 1970 Phys. Rev. Lett. 25 585-7

[268] Kisker E, Schröder K, Campagna M and Gudat W 1984 Phys. Rev. Lett. $\mathbf{5 2} 2285-8$

[269] Fano U 1969 Phys. Rev. 178 131-6

[270] Pierce D T and Meier F 1976 Phys. Rev. B 13 5484-500

[271] Liu W, Chen Y, Lu W, Moy A, Poelker M, Stutzman M and Zhang S 2016 Appl. Phys. Lett. 109252104
[272] Eyers A, Schäfers F, Schönhense G, Heinzmann U, Oepen H P, Hünlich K, Kirschner J and Borstel G 1984 Phys. Rev. Lett. 52 1559-62

[273] Schönhense G 1980 Phys. Rev. Lett. 44 640-3

[274] Heinzmann U, Schönhense G and Kessler J 1979 Phys. Rev. Lett. 42 1603-5

[275] Bychkov Y A and Rashba E I 1984 JETP Lett. 39 78-81

[276] LaShell S, McDougall B A and Jensen E 1996 Phys. Rev. Lett. 77 3419-22

[277] Hsieh D, Qian D, Wray L, Xia Y, Hor Y S, Cava R J and Hasan M Z 2008 Nature 452 970-4

[278] Bradlyn B, Elcoro L, Cano J, Vergniory M G, Wang Z, Felser C, Aroyo M I and Bernevig B A 2017 Nature 547 298-305

[279] Roth C, Hillebrecht F U, Park W G, Rose H B and Kisker E 1994 Phys. Rev. Lett. 73 1963-6

[280] Klebanoff L E, Van Campen D G and Pouliot R J 1994 Phys. Rev. B 49 2047-57

[281] Johnson P D 1997 Rep. Prog. Phys. 60 1217-304

[282] Stryganyuk G et al 2012 Japan. J. Appl. Phys. 51016602

[283] Ueda S, Mizuguchi M, Kojima T, Ishimaru S, Tsujikawa M, Shirai M and Takanashi K 2014 Appl. Phys. Lett. 104 132402

[284] Kozina X et al 2016 J. Electron Spectrosc. Relat. Phenom. 211 $12-8$

[285] Suga S and Tusche C 2015 J. Electron Spectrosc. Relat. Phenom. 200 119-42

[286] Schönhense G et al 2017 Ultramicroscopy 183 19-29

[287] Pietzsch A et al 2008 New J. Phys. 10033004

[288] Oura M et al 2014 Trans. Mater. Res. Soc. Japan 39 469-73

[289] Oura M, Osawa H, Tamasaku K, Tanaka K, Pincelli T, Fujii J, Panaccione G and Tanaka Y 2018 Synchrotron Radiat. News 31 36-41

[290] Spencer B F et al 2013 Phys. Rev. B 88195301

[291] Neppl S et al 2014 Faraday Discuss. 171 219-41

[292] Shavorskiy A et al 2013 AIP Conf. Proc. 1525 475-9

[293] Sezen H, Ozbay E, Aktas O and Suzer S 2011 Appl. Phys. Lett. 98111901

[294] Pressacco F, Sangalli D, Uhlî́r V, Kutnyakhov D, Arregi J, Agustsson S, Brenner G, Redlin H, Heber M, Vasilyev D, Demsar J, Schönhense G, Gatti M, Marini A, Wurth W and Sirotti F 2021 Subpicosecond metamagnetic phase transition driven by non-equilibrium electron dynamics (arXiv:2102.09265 [cond-mat.str-el])

295] Kozina X et al 2011 Phys. Rev. B 84054449

[296] Batterman B W 1964 Phys. Rev. A 133 759-A764

[297] Eng P J et al 1988 Nucl. Instrum. Methods Phys. Res. A 266 $210-4$

[298] Clausnitzer M, Pauly T and Materlik G 1989 Rev. Sci. Instrum. 602436

[299] Zegenhagen J 2018 Applications of the X-Ray Standing Wave Technique in Physical Science Research Synchrotron Light Sources and Free-Electron Lasers (Berlin: Springer) pp 1-31

[300] Emery J D, Detlefs B, Karmel H J, Nyakiti L O, Gaskill D K, Hersam M C, Zegenhagen J and Bedzyk M J 2013 Phys. Rev. Lett. 111215501

[301] Conrad M, Rault J, Utsumi Y, Garreau Y, Vlad A, Coati A, Rueff J P, Miceli P F and Conrad E H 2017 Phys. Rev. B 96 195304

[302] Entani S, Honda M, Naramoto H, Li S and Sakai S 2021 Surf. Sci. 704121749

[303] Thiess S, Lee T L, Aruta C, Lin C T, Venturini F, Brookes N B, Cowie B C C and Zegenhagen J 2015 Phys. Rev. B 92 075117

[304] Schwarz M et al 2017 ACS Nano 11 9151-61 
[305] Brülke C, Heepenstrick T, Humberg N, Krieger I, Sokolowski M, Weiß S, Tautz F S and Soubatch S 2017 J. Phys. Chem. C 121 23964-73

[306] Woicik J C et al 2001 Phys. Rev. B 64125115

[307] Kim C Y, Bedzyk M J, Nelson E J, Woicik J C and Berman L E 2002 Phys. Rev. B 66085115

[308] Thiess S, Lee T-L, Bottin F and Zegenhagen J 2010 Solid State Commun. 150 553-6

[309] Gray A X et al 2010 Phys. Rev. B 82205116

[310] Yang S-H, Gray A X, Kaiser A M, Mun B S, Sell B C, Kortright J B and Fadley C S 2013 J. Appl. Phys. 113 073513

[311] Döring S et al 2011 Phys. Rev. B 83165444

[312] Conlon C S et al 2019 J. Appl. Phys. 126075305

[313] Greer A A et al 2012 Appl. Phys. Lett. 101202402

[314] Nemšák S et al 2014 Nat. Commun. 55441

[315] Karslıoğlu O et al 2015 Faraday Discuss. $18035-53$

[316] Marinova M et al 2015 Nano Lett. 15 2533-41

[317] Martins H et al 2020 arXiv:2012.07993 [cond-mat.mtrl-sci]

[318] Weinen J et al 2015 J. Electron Spectrosc. Relat. Phenom. 198 6-11

[319] Regoutz A et al 2016 Chem. Phys. Lett. 647 59-63

[320] Takegami D et al 2019 Phys. Rev. B 99165101

[321] Trzhaskovskaya M B, Nefedov V I and Yarzhemsky V G 2001 At. Data Nucl. Data Tables 77 97-159

[322] Trzhaskovskaya M B, Nefedov V I and Yarzhemsky V G 2002 At. Data Nucl. Data Tables 82 257-311

[323] Trzhaskovskaya M B, Nikulin V K, Nefedov V I and Yarzhemsky V G 2006 At. Data Nucl. Data Tables 92 245-304

[324] Cooper J W 1993 Phys. Rev. A 47 1841-51

[325] Sekiyama A et al 2010 New J. Phys. 12043045

[326] Sekiyama A, Higashiya A and Imada S 2013 J. Electron Spectrosc. Relat. Phenom. 190 201-4

[327] Fujiwara H et al 2016 J. Synchrotron Radiat. 23 735-42

[328] Ueda S and Hamada I 2017 J. Phys. Soc. Japan. 86124706

[329] Nakatsu Y et al 2011 Phys. Rev. B 83115120

[330] Ouardi S, Fecher G H and Felser C 2013 J. Electron Spectrosc. Relat. Phenom. 190 249-67

[331] Elmers H J et al 2013 Phys. Rev. B 88174407

[332] Dorney K M et al 2019 Nat. Photon. 13 123-30

[333] Lee J C T, Alexander S J, Kevan S D, Roy S and McMorran B J 2019 Nat. Photon. 13 205-9

[334] Seiboth F et al 2019 Opt. Lett. 444622

[335] De Ninno G et al 2020 Nat. Photon. 14 554-8

[336] Piancastelli M N, Marchenko T, Guillemin R, Journel L, Travnikova O, Ismail I and Simon M 2020 Rep. Prog. Phys. 83016401

[337] Oura M, Gejo T, Nagaya K, Kohmura Y, Tamasaku K, Journel L, Piancastelli M N and Simon M 2019 New J. Phys. 21 043015

[338] Cederbaum L S, Tarantelli F, Sgamellotti A and Schirmer J 1986 J. Chem. Phys. 85 6513-23

[339] Koulentianos D et al 2018 J. Chem. Phys. 149134313

[340] Püttner R et al 2015 Phys. Rev. Lett. 114093001

[341] Goldsztejn G et al 2016 Phys. Rev. Lett. 117133001

[342] Feifel R et al 2017 Sci. Rep. 713317

[343] Koulentianos D et al 2018 Phys. Chem. Chem. Phys. 20 2724-30

[344] Marchenko T et al 2020 J. Phys. B: At. Mol. Opt. Phys. 53 224002

[345] Carniato S et al 2016 Phys. Rev. A 94013416

[346] Travnikova O et al 2016 Phys. Rev. Lett. 116213001

[347] Travnikova O et al 2017 Phys. Rev. Lett. 118213001

[348] Marchenko T et al 2018 Phys. Rev. A 98063403

[349] Žitnik M et al 2016 Phys. Rev. A 93021401

[350] Marchenko T et al 2017 Phys. Rev. Lett. 119133001

[351] Simon M et al 2014 Nat. Commun. 54069

[352] Kukk E et al 2018 Phys. Rev. Lett. 121073002
[353] Céolin D et al 2019 Proc. Natl Acad. Sci. USA 116 4877-82

[354] Takata Y et al 2007 Phys. Rev. B 75233404

[355] Guillemin R et al 2015 Phys. Rev. A 92012503

[356] Kosugi S et al 2020 Phys. Rev. Lett. 124183001

[357] Siegbahn H and Siegbahn K 1973 J. Electron Spectrosc. Relat. Phenom. 2 319-25

[358] Siegbahn K 1985 J. Electron Spectrosc. Relat. Phenom. 36 113-29

[359] Starr D E, Liu Z, Hävecker M, Knop-Gericke A and Bluhm H 2013 Chem. Soc. Rev. 42 5833-57

[360] Trotochaud L, Head A R, Karslioğlu O, Kyhl L and Bluhm H 2017 J. Phys. Condens. Matter 29053002

[361] Salmeron M 2018 Top. Catal. 61 2044-51

[362] Tougaard S and Greiner M 2020 Appl. Surf. Sci. 530 147243

[363] Amann P et al 2019 Rev. Sci. Instrum. 90103102

[364] García G, Roteta M and Manero F 1997 Chem. Phys. Lett. 264 589-95

[365] Parratt L G 1954 Phys. Rev. 95 359-69

[366] Favaro M, Abdi F, Crumlin E, Liu Z, van de Krol R and Starr D 2019 Surfaces 2 78-99

[367] Frank Ogletree D, Bluhm H, Hebenstreit E D and Salmeron M 2009 Nucl. Instrum. Methods Phys. Res. A 601 151-60

[368] Müller M, Nemšák S, Plucinski L and Schneider C M 2016 Functional materials for information and energy technology: insights by photoelectron spectroscopy J. Electron Spectrosc. Relat. Phenom. 208 24-32

[369] Maibach J, Xu C, Eriksson S K, Åhlund J, Gustafsson T, Siegbahn H, Rensmo H, Edström K and Hahlin M 2015 Rev. Sci. Instrum. 86044101

[370] Takagi Y et al 2014 Appl. Phys. Lett. 105131602

[371] Takagi Y et al 2017 Phys. Chem. Chem. Phys. 19 6013-21

[372] Takagi Y et al 2017 Appl. Phys. Express 10076603

[373] Yu L, Takagi Y, Nakamura T, Sakata T, Uruga T, Tada M, Iwasawa Y, Masaoka S and Yokoyama T 2019 J. Phys. Chem. C 123 603-11

[374] Nakamura T, Takagi Y, Chaveanghong S, Uruga T, Tada M, Iwasawa Y and Yokoyama T 2020 J. Phys. Chem. C 124 17520-7

[375] Axnanda S et al 2015 Sci. Rep. 59788

[376] Hansen W N 1980 Surf. Sci. 101 109-22

[377] Kolb P M, Rath D L, Wille R and Hansen W N 1983 Ber. Bunsengesellschaft Phys. Chem. 87 1108-13

[378] Ning Y, Fu Q, Li Y, Zhao S, Wang C, Breitschaft M, Hagen S, Schaff O and Bao X 2019 Ultramicroscopy 200 105-10

[379] Sowinska M, Bertaud T, Walczyk D, Thiess S, Calka P, Alff L, Walczyk C and Schroeder T 2014 J. Appl. Phys. 115 204509

[380] Meunier B, Martinez E, Rodriguez-Lamas R, Pla D, Burriel M, Boudard M, Jiménez C, Rueff J-P and Renault O 2019 J. Appl. Phys. 126225302

[381] Arndt B et al 2017 Adv. Funct. Mater. 271702282

[382] Kröger E, Petraru A, Quer A, Soni R, Kalläne M, Pertsev N A, Kohlstedt H and Rossnagel K 2016 Phys. Rev. B 93 235415

[383] Gueye I, Le Rhun G, Renault O, Cooper D, Ceolin D, Rueff J P and Barrett N 2017 Appl. Phys. Lett. 111032906

[384] Ikeuchi J, Hamamatsu H, Miyamoto T, Tanaka S, Yamashita Y, Yoshikawa H and Ueda S $2015 \mathrm{~J}$. Appl. Phys. 118 085308

[385] Kiuchi H, Hikima K, Shimizu K, Kanno R, Toshiharu F and Matsubara E 2020 Electrochem. Commun. 118106790

[386] Matveyev Y et al 2019 Nanoscale 11 19814-22

[387] Matveyev Y, Negrov D, Chernikova A, Lebedinskii Y, Kirtaev $\mathrm{R}$, Zarubin S, Suvorova E, Gloskovskii A and Zenkevich A 2017 ACS Appl. Mater. Interfaces 9 43370-6

[388] Koch R J, Jozwiak C, Bostwick A, Stripe B, Cordier M, Hussain Z, Yun W and Rotenberg E 2018 Synchrotron Radiat. News 31 50-2 
[389] Beaulieu S et al 2020 Phys. Rev. Lett. 125216404

[390] Jansen G S M et al 2020 New J. Phys. 22063012

[391] Wallauer R, Raths M, Stallberg K, Münster L, Brandstetter

D, Yang X, Güdde J, Puschnig P, Soubatch S, Kumpf C,

Bocquet F C, Tautz F S and Höfer U 2021 Tracing orbital

images on ultrafast time scales Science 371 1056-9

[392] Kiss T et al 2008 Rev. Sci. Instrum. 79023106
[393] Shimojima T, Okazaki K and Shin S 2015 J. Phys. Soc. Japan. 84072001

[394] Tamai A, Meevasana W, King P D, Nicholson C W, De La Torre A, Rozbicki E and Baumberger F 2013 Phys. Rev. B 87075113

[395] Schönhense G, Babenkov S, Vasilyev D, Elmers H-J and Medjanik K 2020 Rev. Sci. Instrum. 91123110 